Regression of cardiac hypertrophy achieved by high dose propranolol treatment in children and adults with hypertrophic cardiomyopathy

\section{I Östman-Smith}

Cardiac Department, John Radcliffe Hospital, Oxford

High dose oral propranolol treatment $(7-18 \mathrm{mg} / \mathrm{kg} /$ day $)$ is being used to treat a series of patients with hypertrophic cardiomyopathy. The group now comprises eight infants who presented with congestive heart failure and failure to thrive or syncope, or both; four children with progressive worsening of hypertrophic cardiomyopathy or severe ventricular arrhythmias, or both; and three adults with angina and near syncope or severe ventricular arrhythmias, or both. The group has been treated for a total of 68 patient years with no deaths; all patients are symptom free except the most recently recruited adult. Two infants presenting with severe symptomatic hypertrophic cardiomyopathy have remianed well over a 10 year follow up. All 15 patients have shown electrocardiographic and echocardiographic evidence of regression of pathological cardiac hypertrophy. Because 12 of the patients are still growing echocardiographic measurements have included not only absolute measurements of wall thickness and cavity dimensions but also calculation of diastolic and systolic ratios of wall thickness to cavity diameter. The observations were compared to pretreatment values in the same patient. In 12 patients treated for at least one year the Wilcoxon signed rank test showed that high dose propranolol produced a significant reduction in absolute septal thickness $(-24 \% ; \mathrm{p}<0.01)$; diastolic septum to cavity ratio $(-38 \%) ; \mathrm{p}<0.0025)$; posterior left ventricular wall hypertrophy, when present $(-44 \%$; $<<0.013)$; systolic left ventricular wall to cavity ratio $(-37 \% ; p<0.01)$; and a reduction in ejection fraction towards the normal range $(-11 \% ; p<0.013)$. The serum propranolol concentrations required to produce regression of hypertrophy were $150-1360 \mu \mathrm{g} / \mathrm{l}$ ("normal" therapeutic range $50-100 \mu \mathrm{g} / \mathrm{l})$. Five patients needed the addition of disopyramide to control their arrhythmias.

It is concluded that high dose propranolol abolishes symptoms and greatly improves survival in infants with symptomatic hypertrophic cardiomyopathy and that this treatment can induce regression of hypertrophy in both children and adults with this condition if care is taken to achieve a profound 24 hour $\beta$ adrenoceptor blockade.

Endothelin may be a circulating vasoconstrictor in chronic heart failure

J McMurray, S G Ray, J J Morton, H J Dargie Department of Cardiology, Western Infirmary, Glasgow, and Medical Research Council Blood Pressure Unit, Glasgow

Endothelins (ET) are potent long acting vasoconstrictor peptides. As chronic heart failure is characterised by reduced peripheral and renal perfusion we measured ET concentrations in patients with this syndrome with a previously described assay. Ten healthy controls aged 52 (3) years (three women) were compared with 12 patients aged 64 (5) years (all men) with chronic heart failure (three New York Heart Association class IV; eight class III, one class
II) treated with diuretics, digoxin, and vasodilators. The mean plasma ET concentration in controls was 5.9 (SEM $0.2) \mathrm{pmol} / 1 \mathrm{compared}$ with $13.6(1.0) \mathrm{pmol} / 1$ in patients $(\mathrm{p}<0.01)$. Eight patients exercised on a treadmill. Plasma ET concentrations did not increase further with maximum exercise (11.4 (1.6) before exercise $v 10.5(1.6)$ afterwards). In a second study 13 patients aged 29-70 years (four men) with chronic heart failure were investigated at the time of cardiac catheterisation. Significant renal extraction of ET was found: the concentration of ET in the aorta was $11 \cdot 1$ $(0.8) \mathrm{pmol} / \mathrm{l}$ and in the renal vein $8.8(0.6) \mathrm{pmol} / 1$ $(\mathrm{p}=0.02)$. Thus ET concentrations are raised in chronic heart failure either from increased secretion or from decreased clearance. Increased secretion may occur to maintain arterial pressure in the face of a limited cardiac reserve; however, the cardiovascular stress of upright exercise did not further increase plasma concentrations. Reduced clearance, due to poor renal perfusion, could also account for the raised plasma ET concentration in heart failure as these results show, for the first time, that ET is extracted by the kidneys.

Vasoconstriction is pathophysiologically important in heart failure and raised ET concentrations may contribute to this.

\section{Improved lymphocyte $\beta$ receptor function and cardiac pumping capacity after converting enzyme inhibition in heart failure}

\section{J N Townend, S J S Virk, F X Oiang, N Lawson, W A Littler, $M$ K Davies \\ Department of Cardiovascular Medicine, University of Birmingham}

Cardiac $\beta$ receptor down regulation in heart failure causes reduced sensitivity to $\beta$ agonists. We have previously showed that lymphocyte $\beta$ receptor (LBR) down regulation in heart failure can be partially reversed by captopril. We studied 12 patients with severe heart failure (New York Heart Association class III/IV) before and after 16 weeks of oral quinapril treatment to determine whether LBR up regulation in response to an angiotensin converting enzyme inhibitor is associated with a concomitant improvement in cardiac $\beta$ receptor function. This was assessed by measuring invasive haemodynamic variables during bicycle exercise and incremental $\beta$ agonist (dobutamine) infusion. After quinapril LBR function (in vitro isoprenaline stimulated lymphocyte cAMP production) improved significantly: at $15 \mathrm{mmol} / \mathrm{l}$ the cAMP concentration was $376 \%$ of control values after quinapril $v 211 \%$ before quinapril, $\mathrm{p}<0.02$. There were associated increases in cardiac index ( 3.8 to $4.41 / \mathrm{min} / \mathrm{m}^{2}, \mathrm{p}<0.01$ ), stroke volume (63 to $75 \mathrm{ml}, \mathrm{p}<0.05)$, and cardiac power output $(1.7$ to $2.0 \mathrm{~W}$, $\mathrm{p}<0.05)$ at peak exercise. Similarly, with dobutamine at $10 \mu \mathrm{g} / \mathrm{kg} / \mathrm{min}$ cardiac index increased from 4.0 to $4.61 / \mathrm{min} / \mathrm{m}^{2}$ ( $\left.<<0.05\right)$, stroke volume from 70 to $82 \mathrm{ml}$ $(\mathrm{p}<0.01)$, stroke work index from 0.42 to $0.50 \mathrm{~J} / \mathrm{m}^{2}$ $(\mathrm{p}<0.05)$, and cardiac power output from 1.5 to $1.8 \mathrm{~W}$ $(\mathrm{p}<0.05)$.

We conclude that $\mathrm{LBR}$ up regulation seen in patients with severe heart failure in response to angiotensin converting enzyme inhibitors is associated with an improved cardiac response to exercise and dobutamine consistent with functional myocardial $\beta$ receptor upregulation. 
Dilated cardiomyopathy: high incidence of antimyolemmal antibody mediated cytolytic activity in cases of recent onset

T H Taha, P Mazeika, Celia M Oakley, B Maisch Clinical Cardiology Unit, Hammersmith Hospital and Royal Postgraduate Medical School, London, and Klinikum der Philipps-University, Marburg, Germany

Circulating antimyolemmal antibodies (AMLA) have been detected in about $30 \%$ of patients with chronic dilated cardiomyopathy but their incidence in acute cases is unclear. Over 24 months seven consecutive patients with dilated cardiomyopathy (age 44 (14)) and symptoms for less than six months were studied. All had a full virology and autoantibody screen, cardiac biopsy, and cross sectional echocardiography; fractional shortening for the group was $12 \%(4 \%)$. The presence of circulating AMLA was assessed in vitro with intact rat and human cardiocytes and the indirect immunofluorescence test. Serum cytolytic index for AMLA (half life of cardiocytes in patients serum $\div$ half life in control serum) was measured in a microcytotoxicity assay with living rat cardiocytes as target cells. Data were compared with that in 70 controls evaluated in the same laboratory (normal range $0.92(0.07)$, effective cytolysis $<0 \cdot 75)$. Only one patient had positive viral serology, which was for recent coxsackie B2 infection. Autoantibody screening was negative in all. Light microscopy of biopsy samples showed non-specific appearances in two patients; focal myocarditis was found in one case and healing or healed myocarditis in four cases. The cytolytic index for the group was 0.51 (0.05) (range 0.47-0.6, p < 0.05). Significant binding of $\operatorname{IgG}$, IgM, or polyvalent antibodies to AMLA bound to rat or human cardiocytes was present in all seven; five patients were positive with all three markers, and four of these had strongly positive binding on at least one test.

Circulating AMLA are commonly present in dilated cardiomyopathy of recent onset. Their detection might reflect ongoing disease activity, give insight into the immunopathogenesis, and may help identify patients likely to benefit from immunosuppressive treatment.

\section{Cardiac abnormalities associated with HIV infection}

\author{
A J Jacob, G R Sutherland, A G Bird, R P Brettle, \\ N A Boon \\ Royal Infirmary and Western General and City \\ Hospitals, Edinburgh
}

To determine the prevalence of cardiac abnormalities for the first time in a United Kingdom based population infected with HIV 74 patients ( 53 male, 21 female) infected with HIV for between 32 and 87 months were recruited as part of a prospective echocardiographic study. They comprised the two major risk groups for HIV infection: intravenous drug abusers ( 57 patients, 36 male, 21 female; mean age 28.9 years; mean CD4 count 205 cells $/ \mathrm{mm}^{3}$ (normal range $500-1500$ cells $/ \mathrm{mm}^{3}$ )) and homosexuals (17 patients; mean age 34.2 years; mean CD4 count 38 cells $/ \mathrm{mm}^{3}$ ). Ventricular size and function were estimated by standard cross sectional and $M$ mode echocardiographic techniques. Detailed analysis was undertaken by two independent observers. Seven $(9 \cdot 5 \%)$ patients (five homosexuals, two intravenous drug abusers) had global left ventricular dysfunction. Their mean CD4 count was 32 cells $/ \mathrm{mm}^{3}$, indicating end stage disease. There was an excess of homosexuals in this group $(29 \%$ affected $v 4 \%$ of intravenous drug abusers), possibly a reflection of their more advanced HIV disease. Left ventricular dilatation (end diastolic diameter $>58 \mathrm{~mm}$ ) without evidence of dysfunction was found in a further five $(6.8 \%)$ patients (mean CD4 count 227 cells $/ \mathrm{mm}^{3}$ ), all intravenous drug abusers. These abnormalities were related neither to infection with toxoplasma (as judged by the toxoplasma dye test) nor to duration of treatment with the potentially cardiotoxic agent zidovudine (mean 16.3 months). Isolated right ventricular dilatation was found in seven (9.5\%) patients (mean CD4 count 153 cells $/ \mathrm{mm}^{3}$ ) and occurred exclusively in intravenous drug abusers.

In conclusion, we found a high prevalence of dilated cardiomyopathy in HIV infection. This occurs in both major risk groups and seems to be a feature of end stage disease. It may be preceded by left ventricular dilatation. Isolated enlargement of the right ventricle may be a function of intravenous drug abuse rather than HIV infection.

\section{Tumour necrosis factor may contribute to development of "cardiac cachexia"}

\section{J McMurray, I Abdullah, D Shaprio, H J Dargie Departments of Cardiology and Pathological Biochemistry, Western Infirmary, Glasgow}

A significant proportion of patients with chronic heart failure suffer generalised wasting, which in its most extreme form is referred to as "cardiac cachexia." Inadequate nutritional intake is not thought to be the major aetiological factor. Instead it is suggested that a catabolic state is brought about through the actions of a circulating factor; a polypeptide with such actions has been identified and named tumour necrosis factor (TNF). We have measured plasma TNF concentrations in 26 consecutive patients admitted for investigation or treatment, or both, of chronic heart failure (due to coronary artery disease in 17, valve disease in seven, dilated cardiomyopathy in one, and congenital heart disease in one). The condition of all patients was New York Heart Association class III or IV and was treated with diuretics; most patients also received digoxin and an angiotensin converting enzyme inhibitor. Once treatment was optimised subcutaneous fat was measured (fat content rather than weight was measured to circumvent the potential problem of residual fluid retention). Using calipers a single observer measured bicep, tricep, infrascapular, and suprailiac skinfold thickness in quadruplicate (skinfolds over the lower part of the body were avoided because of the possibility of dependent residual fluid retention). Percentage body fat content was derived from the sum of the mean of each of these skinfold measurements by using standard tables. Patients were prospectively grouped as "cachectic" (male $<27 \%$, females $<29 \%$ body fat) or "not cachectic." TNF was measured in venous blood "blind" to group allocation with a sensitive radioimmunoassay. Sixteen patients (11 male) were cachectic (mean body fat content $21 \%$ (SD $4 \cdot 8 \%$ )). Of these, nine had a raised $(>15 \mathrm{pg} / \mathrm{ml})$ concentration of TNF. Mean TNF in this group was 74 (SEM 20) $\mathrm{pg} / \mathrm{ml}$. Ten patients were not cachectic (body fat $31 \%(3.3 \%)$ ); only one had a raised TNF concentration, which was $22 \mathrm{pg} / \mathrm{ml}$ (difference between groups, $\mathrm{p}<0.001$ ). TNF was unmeasurable $(<15 \mathrm{pg} / \mathrm{ml})$ in plasma from five normal subjects.

TNF causes cachexia in experimental animals and catabolism in humans; it may also contribute to wasting in chronic heart failure. TNF has other actions, such as promoting clotting and inducing anaemia, which may also be undesirable in chronic heart failure. 
Blood flow patterns at arterial bifurcations depend on vasomotor tone and heart rate

C J H Jones, M J Lever, K H Parker, F Kajiya Imperial College, London, and Kawasaki Medical School, Kawasaki, Japan

Vasomotor regulation by endothelium derived relaxing factor depends on pulsatile blood flow patterns, which depend on arterial tone and heart rate. The effects of vasoactive drugs and pacing on flow were studied by $20 \mathrm{MHz}$ Doppler velocimetry to measure velocity profiles and waveforms at exposed bifurcations in eight paced anaesthetised dogs. A calcium antagonist, YM-09730-5 (50 $\mu \mathrm{g})$, increased the peak velocity ( $+25 \%$ (SD $9 \%$ ), p < 0.05 ) and duration $(+48 \%(6 \%), \mathrm{p}<0.05)$ of forward flow and reduced peak reverse flow velocity $(-17 \%(5 \%)$, $\mathrm{p}<0.05)$. Conversely, angiotensin II $(1 \mu \mathrm{g} / \mathrm{min})$ reduced the peak velocity $(-21 \%(5 \%), p<0.05)$ and duration $(-23 \%(4 \%), p<0.05)$ of forward flow. Glyceryl trinitrate $(150 \mu \mathrm{g} / \mathrm{min})$ reduced peak forward velocity $(-14 \%(4 \%), \mathrm{p}<0.05)$ but prolonged both forward $(+27 \%(6 \%), \mathrm{p}<0.05)$ and reverse flow $(39 \%(7 \%)$, $\mathrm{p}<0.05)$. Peak velocity and duration of forward and reverse flow were all greater when the dogs were paced at $1 \mathrm{~Hz}$ rather than $2 \mathrm{~Hz}$ (forward flow $52(22) v 40(16) \mathrm{cm} / \mathrm{s}$ and $131(40) v 119(28) \mathrm{ms}$; reverse flow $-19(8) v-15$ (7) $\mathrm{cm} / \mathrm{s}$ and $145(30) v 115(25) \mathrm{ms} ; \mathrm{p}<0.05)$. Changes in velocity wave forms were accompanied by changes in velocity profiles.

Both vasoactive drugs and heart rate changes alter arterial bifurcation blood flow patterns in intact animals and may thus alter release of endothelium derived relaxing factor.

\section{Energy wave generation by the left ventricle:} influence of contractility and vasomotor tone

\section{J H Jones, K H Parker, M Sugawara}

Imperial College, London, and Tokyo Women's Medical College, Tokyo, Japan

Aortic flow is dominated by accelerating and decelerating waves generated by the left ventricle $(\mathrm{LV})$, detectable as positive peaks of wave intensity, $\mathrm{dP} / \mathrm{dU}(\mathbf{P}$, pressure and $U$, velocity) in early and late systole (compression and expansion waves respectively). As vasomotor tone and LV contraction influence aortic flow differently, we calculated aortic $\mathrm{dP} / \mathrm{dU}$ in 13 anaesthetised closed chested dogs before and during pharmacological inotropic $(n=6)$ and vasomotor $(n=7)$ manipulation. Peak LV compression wave intensity, causing aortic acceleration, was increased by dobutamine $15 \mu \mathrm{g} / \mathrm{kg} / \mathrm{min}$ (mean change $+146 \%$ (SEM $17 \%), \mathrm{p}<0.01$ ) and decreased by propranolol $10 \mathrm{mg}$ $(-59 \%(5 \%), p<0.01)$. However, the compression wave was also dependent on vasomotor tone as its peak intensity was reduced by vasoconstriction with methoxamine $2 \mathrm{mg}$ $(-62 \%(6 \%), p<0.01)$. Peak LV expansion wave intensity, causing late systolic deceleration, was unaltered by inotropic manipulation or by vasoconstriction but was reduced by vasodilatation with glyceryl trinitrate $0.02 \mathrm{mg} / \mathrm{kg} / \mathrm{min}(-25 \%(8 \%), \mathrm{p}<0.01)$, due to the delayed arrival in the aorta of reflected waves with negative wave intensity.

Thus LV compression wave amplitude indicates LV contractile state but depends also on vasomotor tone, being decreased by vasoconstriction. Late systolic aortic flow deceleration depends more on vasomotor tone than the contractile state of the LV.
Rapid oxidation of plasma low density lipoprotein fraction in coronary artery disease

S W Davies, V K Datta, A D Timmis, R Balcon, C M Rice-Evans, K R Bruckdorfer

Royal Free Hospital Medical School and London Chest Hospital, London

Even under normal circumstances there are large fluxes of low density lipoproteins (LDL) through the intima. Evidence from animal experiments and from cell culture in vitro indicates that when LDL are modified by oxidation they are still taken up by macrophages but cannot be further processed, so that they become foam cells. Thus oxidised LDL may lead to atheroma, and the susceptibility of LDL to oxidation may be as important for atherogenesis as the absolute concentrations of LDL. We therefore determined whether plasma LDL is more readily oxidisable in patients with coronary artery disease. Venous blood samples were obtained from 10 patients aged 43-67 with coronary artery disease, and from 10 controls. The LDL fraction was separated by density gradient centrifugation (1.019-1.063) and dialysis. During incubation with copper ions the rates of $(a)$ disappearance of carotene, $(b)$ appearance of conjugated dienes, and $(c)$ appearance of lipid peroxides were monitored spectrophotometrically. LDL oxidised more rapidly in patients with coronary artery disease than they did in controls $(p<0.02)$.

Increased oxidisability of plasma LDL may contribute to atheroma in patients with coronary artery disease. Practical implications are (a) that drugs protecting LDL against oxidation may slow or prevent atherogenesis and (b) that measurements in young subjects might identify those at high risk of developing future coronary artery disease.

\section{Direction of ventricular activation influences timing of repolarisation}

P I Taggart, P M Sutton, R M John, M J Lab, S Horner, R H Swanton

Department of Cardiology, Middlesex Hospital; Department of Physiology, University College and Middlesex School of Medicine; and Department of Physiology, Charing Cross Hospital, London

Ventricular arrhythmias are frequently initiated by an ectopic focus acting as a trigger. The reason why some ventricular ectopic beats or even short runs of ventricular tachycardia are benign is unclear. One possible mechanism may be the effect of an alteration in the direction of activation on the timing of repolarisation. To assess this possible mechanism we used the monophasic action potential (MAP) as a measure of repolarisation. The direction of activation was altered between one atrial and two ventricular sites. Atrioventricular (AV) sequential pacing was incorporated to minimise any possible effects of loss of atrial contribution to ventricular filling. Twelve patients (10 men; aged 50-66 years) were studied. Four catheter electrodes were positioned: (a) MAP on left ventricular lateral wall, $(b) \mathrm{MAP}$ on right ventricular septum, (c) pacing electrode in right ventricular apex, $(d)$ pacing electrode in atrial appendage. Steady state atrial pacing was established and the pacing site sequentially switched in turn from atrium to ventricle to atrium to AV sequential to atrium. The positions of the right ventricular MAP catheter and right ventricular pacing electrode were interchanged and the sequence repeated. Peak systolic pressure changes (mean (SD)) during switching between the different pacing modes were as follows: atrial to ventricular 134 (SD 9) to 100 
(5) $\mathrm{mm} \mathrm{Hg}(\mathrm{p}<0.001)$ and atrial to AV sequential $152(20)$ to 144 (16) $\mathrm{mm} \mathrm{Hg}$ (NS). The MAP duration altered in all patients, with the altered pacing sequence either lengthening or shortening. The mean changes in MAP duration associated with alteration in pacing orientation between atrial to ventricular and atrial to AV sequential were similar, being 5.6 $(5 \cdot 1) \mathrm{ms}$ and $6 \cdot 3(4 \cdot 5) \mathrm{ms}$ respectively (NS). The MAP changes were therefore primarily a function of altered direction of activation as opposed to pressure changes. In one patient with severe left ventricular dysfunction pacing from the apex of the right ventricle reproducibly induced prolongation of the left ventricular MAP by $40 \mathrm{~ms}$ with electrical alternans of $30 \mathrm{~ms}$ whereas pacing from the right ventricular septum failed to do so.

These preliminary results suggest that altering the site of ventricular activation alters the timing of repolarisation in the right and left ventricles. Although electrotonic interactions cannot be excluded, such findings accord with the hypothesis that changing the sequence of contraction of the ventricular fibres and thereby altering the stress or strain between neighbouring fibre groups may induce electrophysiological effects sufficient to generate clinically significant arrhythmias.

\section{Mortality in outpatients awaiting coronary arteriography}

\section{S W Davies, P J Timmins, C S Walker, A D Timmis, M T Rothman, C A Layton, R Balcon \\ London Chest Hospital, London}

Facilities for coronary arteriography are limited in the United Kingdom, and correct selection of patients for this investigation is important to make best use of the resources available. It is equally important to consider the delays involved and to identify patients at high risk of cardiac events who require urgent investigation. At a cardiac centre with on site surgery the waiting list for coronary arteriography was audited over a four month period. During this time 934 cardiac catheterisation procedures were performed, including 592 coronary arteriograms for ischaemic heart disease (138 patients were transferred from other hospitals, 101 from home on the urgent waiting list, and 353 from home on the routine waiting list). Outpatients were assigned by a consultant cardiologist to the urgent waiting list (delay 0-3 weeks, mean 1 week) or to the routine waiting list (delay 3-12 weeks, mean 4.5 weeks). During the four month period seven patients died at home while awaiting admission for arteriography. One patient died two weeks after entry on to the urgent list, corresponding to $0.9 \%$ of admissions from the urgent list over the four months. Six patients died 3-9 (mean 6) weeks after entry on to the routine list, corresponding to $1.7 \%$ of routine admissions. Patients who died were aged 45-67 with angina grades II-IV; two were diabetic and one had mild heart failure. The mode of death ascertained from the patient's general practitioner was sudden collapse in three and death after admission to hospital with presumed infarction in four. An independent observer reviewing referral letters would have reassigned one of the six who died on the routine list to the urgent list. Over the past three years the procedural mortality from coronary arteriography has been $0.086 \%$.

This audit shows a small but definite mortality among patients awaiting coronary arteriography which exceeds the risks of the procedure itself. Furthermore, these patients cannot easily be identified from clinical details at the time of referral, suggesting that this mortality can be reduced only by shortening waiting lists.

\section{Geriatric congenital heart disease: incidence of} initial presentation

P J Scott, A R Essop, G A Wharton, G J Williams

Non-invasive Heart Unit, Killingbeck Hospital, Leeds

Initial presentation of congenital heart disease in elderly people is well recognised, but information on incidence is scarce. Over the past five years 3850 patients over 65 were referred to our unit for echocardiography. Nineteen $(0.5 \%)$ of these presenting for the first time with symptoms and signs of cardiac disease were found to have a congenital heart defect. Patients were aged 65 to 87 years. The condition of five patients was New York Heart Association (NYHA) class I, of four class II, of eight class III, and of two class IV. Eleven were referred because of unexplained heart failure, four with a systolic murmur for clarification, and in four cases the condition had been correctly diagnosed clinically before referral. Thirteen patients were in sinus rhythm and six in atrial fibrillation. Lesions detected were: atrial septal defect (eight cases), ventricular septal defect (three), ventricular septal defect plus pulmonary valve stenosis (one), bicuspid aortic valve (three), tetralogy of Fallot (two), Ebstein's anomaly of the tricuspid valve (one), and cor triatriatum (one). Four patients, three with atrial septal defect and one with a stenotic bicuspid aortic valve, had corrective surgery and were well at follow up (the disease was NYHA class II in all cases).

A small number of patients, $0.5 \%$ of referrals to us for echocardiography, reach old age with previously undiagnosed congenital heart disease. Some lesions are complex, but despite this a considerable number (nine out of our 19 patients) are still well (NYHA classes I and II) and have only clinically mild heart failure.

\section{Electrocardiogram interpretation in general practice}

W A McCrea, S Saltissi

Cardiac Department, Royal Liverpool Hospital, Liverpool

In the United Kingdom medical prehospital coronary care is almost entirely dependent on the general practitioner (GP) service. There is currently much debate on the role of GPs in initiating thrombolysis outside hospital, thereby reducing the delay in providing this treatment to suitable patients with acute myocardial infarction (AMI). It has been recommended, however, that only general practitioners proficient in interpreting an electrocardiogram (ECG) should administer thrombolytic agents. We therefore decided to assess the current ability of general practitioners to recognise acute ischaemia in an ECG. One hundred and fifty general practitioners in the Merseyside area were selected at random and, without any warning, were approached at clinical meetings or in surgeries and asked to interpret a series of six 12 lead ECGs. Details of age, postgraduate training, and clinical practice were also requested. One hundred and six doctors (mean age 45 years) agreed to participate. Three of the six ECGs showed acute myocardial infarction, one was normal, and two showed non-acute ischaemic changes. Between $8 \%$ and $30 \%$ of general practitioners accurately diagnosed acute myocardial infarction, $82 \%$ recognised a normal ECG, and between $22 \%$ and $25 \%$ correctly interpreted non-acute ischaemia. Neither routine use of an electrocardiograph nor postregistration hospital experience in general medi- 
cine was associated with significantly greater ability to detect acute myocardial infarction in an ECG.

We conclude that at present the majority of general practitioners are not adequately proficient in interpreting acute ischaemia in ECGs.

\section{Potential reduction in risk of sudden death by implantable defibrillators: economic implications}

\author{
R Hendriks, A P Rae, S M Cobbe \\ Department of Medical Cardiology, Royal Infirmary, \\ Glasgow
}

The implantable cardioverter-defibrillator (ICD) is effective at preventing sudden death in patients with sustained ventricular tachycardias (VT). At present, rates of funding and implantation in the United Kingdom are severely restricted. We undertook a retrospective review of 127 patients investigated and managed for sustained VT between 1984-90 to assess the potential benefits of and requirements for ICD implantation. Patients experiencing VT within 48 hours of myocardial infarction were excluded. Eleven patients (group 1) had no sustained VT inducible at baseline electrophysiological study. Eighty five patients had inducible VT, of whom 30 (group 2) were receiving drug suppression treatment; of the 55 whose VT was still inducible, 44 had a haemodynamically tolerated arrhythmia (group 3) and 11 had a poorly tolerated arrhythmia (group 4) despite drug treatment. Empirical treatment without electrophysiological study was used in 31 (group 5). During a mean follow up of 18 months (range $0 \cdot 2-76$ ), there were 17 sudden deaths. The incidence in groups $1-5$ respectively was $0 \%, 10 \%, 16 \%, 36 \%$, and $10 \%$. The number of implants required per sudden death prevented and the costs according to different policies were calculated assuming a device cost of $£ 12000$. This would be: inducible on treatment/poorly tolerated $11 / 4(£ 33000)$, all inducible on treatment (groups 3 and 4) 55/11 ( $£ 60000)$, all inducible at electrophysiological study $86 / 15$ ( $£ 69000)$, and all patients with VT 127/17 ( $£ 90000)$.

Many $(65 \%)$ sudden deaths could be prevented by implanting ICDs into groups 3 and 4, but at an initial cost of $£ 660000$.

\section{Does programmed ventricular stimulation identify patients at risk of sudden death in survivors of acute myocardial infarction?}

\section{T G Farrell, V Paul, D E Ward, A J Camm St George's Hospital, London}

Programmed ventricular stimulation was performed in 90 survivors of acute myocardial infarction (range 7-14 days). A strict stepwise protocol was used involving stimulation at a single right ventricular site during sinus rhythm and three drive cycles with up to three extra stimuli. Stimulation was terminated on the induction of a sustained arrhythmia. Ten patients developed sustained monomorphic ventricular tachycardia (SMVT); 24 patients developed polymorphic ventricular tachycardia/ ventricular fibrillation (PMVT) and in 56 patients no sustained arrhythmia was induced. In 25 patients nonsustained ventricular tachycardia (NSVT), defined as greater than six beats duration but less than 30 seconds, was induced. Fourteen patients with NSVT progressed to PMVT at the same or a later stage of the protocol, but no patient with NSVT progressed to SMVT. During a one year follow up there were five sudden deaths and five patients re-presented with sustained ventricular tachycardia (VT). The induction of SMVT at programmed ventricular stimulation identified all five cases of VT (sensitivity $100 \%$, specificity $94 \%$, positive predictive accuracy $50 \%$ ) but only two cases of sudden death (sensitivity $40 \%$, specificity $91 \%$, positive predictive accuracy $20 \%$ ). Those patients developing VT were characterised by poor left ventricular function and increased age compared with the patients who died suddenly. Although programmed ventricular stimulation failed to identify sudden death, other markers of arrhythmic risk were present in this group; three out of five had a positive signal averaged electrocardiogram and all five had frequent ventricular ectopics $(>10 / \mathrm{h})$.

Programmed ventricular stimulation identifies patients at risk of developing late ventricular tachycardia rather than sudden death. PMVT is a non-specific response and can be avoided by terminating programmed ventricular stimulation if a NSVT occurs, without loss of predictive accuracy.

\section{Heart rate variability and left ventricular ejection fraction: comparison of predictive characteristics for all cause mortality, arrhythmic events, and sudden death after myocardial infarction}

O Odemuyiwa, M Malik, T Farrell, Y Bashir, J Poloniecki, A J Camm

Department of Cardiological Sciences, St George's Hospital, London

To refine postinfarction risk stratification we compared the predictive values of the heart rate variability (HRV) index (calculated as the baseline width of the histogram of RR interval durations) and the ejection fraction (EF) for all cause mortality, arrhythmic events, and sudden death in 385 survivors of acute myocardial infarction followed up for at least three months. Thirty eight $(9.9 \%)$ patients died. There were 30 arrhythmic events, of which 16 were sudden deaths. A HRV index of $<305 \mathrm{~ms}$ had a sensitivity of $75 \%$ and a specificity of $52 \%$ for all cause mortality whereas for a sensitivity of $75 \%$ the EF had a specificity of $40 \%$. A HRV index of $<234 \mathrm{~ms}$ had a sensitivity of $75 \%$ and a specificity of $76 \%$ for arrhythmic events when the EF had a specificity of only $45 \%$. An EF of $\leqslant 40 \%$ had a sensitivity of $42 \%$ and a specificity of $75 \%$ for arrhythmic events; for a sensitivity of $42 \%$ a $\mathrm{HRV}$ index of $156 \mathrm{~ms}$ had a specificity of $92 \%$. To predict $75 \%$ of sudden deaths a HRV index of $<250 \mathrm{~ms}$ had a specificity of $75 \%$ and the EF a specificity of $47 \%$. An EF $\leqslant 40 \%$ had a sensitivity of $40 \%$ and a specificity of $73 \%$ for sudden death; for a sensitivity of $40 \%$ a HRV index of $<190 \mathrm{~ms}$ had a specificity of $83 \%$.

The HRV index seems to be a better predictor of important postinfarction complications than the EF. 


\section{Lipid adnormalities in British Asian and white patients and in Asian patients in India with coronary artery disease}

\author{
J Dhawan, C L Bray, D H Bennett, J P Miller, \\ B Faragher \\ Wythenshawe Hospital, Manchester
}

Lipid profiles which included total cholesterol (TC), triglyceride (TGL), high density lipoprotein (HDL), apo A1, apo $B$, and lipoprotein (a) ( $L p(a)$ ) were measured in a consecutive series of 56 British Asian (BA) patients and 65 randomly selected white patients. These were compared with $47 \mathrm{BA}$ controls and 57 white controls randomly selected from the community. Consecutive series of 28 Asian patients in India (API) were compared with 28 randomly selected Asian controls in India (ACI). All British Asian subjects had migrated from the north west of the Indian subcontinent and subjects resident in India were from the same geographical area. These groups have not previously been compared. Statistical significance was set at a conventional $5 \%$ level. $\mathrm{TC}(\mathrm{mmol} / \mathrm{l})$ concentration was significantly higher in patients than in controls and values were lowest in subjects from India (6.5 $v 6.0$ in white controls, $6.2 v 5.6$ in BA, and 5.5 $v 5.1$ in Asians in India). TGL concentrations were significantly higher in patients than in controls $(1.88 v 1.29$ in white controls, $2.11 v 1.68$ in BA, and $1.92 v 1.39$ in Asians in India). HDL was significantly lower in patients than in controls $(1.09 \mathrm{v} 1.28$ in white controls, $1.05 v 1.09$ in BA, and 0.89 $v 1.24$ in Asians from India). Apo Al concentrations were similar$1.14 v 1.20$ in white controls, $1.13 v 1.23$ in BA, and 0.97 $v 1 \cdot 10$ in Asians from India. Apo B concentrations were significantly higher in patients than in controls (1.55 $v 1.4$ in white controls, $1.42 v 1.09$ in BA, and $1.34 v 1.2$ in Asians from India). $\mathrm{Lp}$ (a) concentrations ( $\mathrm{mg} / \mathrm{dl}$ ) were significantly higher in BA patients but not in white patients (187 $v 114$ and $180 v 162)$ and lower in API than ACI (151 $v 170)$.

We conclude that Asians (patients and controls) have significantly lower TC, higher TGL, lower HDL, lower apo A1, and higher apo B concentrations than white patients and controls. British Asians have significantly higher TC and TGL than Asians in India, probably signifying the effect of environment. $\mathrm{Lp}$ (a) concentrations are interesting in that they are higher in BA than whites but patients had lower concentrations than controls from India.

\section{Haemodynamic effects of total right ventricular disarticulation for ventricular tachycardia}

J C Doig, K Nimkhedkar, J P Bourke, J M McComb, C J Hilton, S S Furniss, R W F Campbell

Academic Cardiology, Freeman Hospital, Newcastle upon Tyne

Total right ventricular disarticulation is a radical operation performed to control ventricular tachycardia in patients with arrhythmogenic right ventricular dysplasia (ARVD). This analysis aims at defining the acute and chronic haemodynamic impact of the operation based on five patients with histologically confirmed ARVD who had such surgery at this hospital (mean age 30 years; four male, one female). After surgery creatine kinase and creatine kinase $\mathrm{MB}$ activities rose to mean values of $2348 \mathrm{IU} / 1$ and $175 \mathrm{U} / 1$ respectively. Acute right heart failure was present in three patients $(60 \%)$ but cleared with intravenous fluids and inotropic treatment. Echocardiographic, nuclear, and angiographic studies showed no right ventricular (RV) movement, but this was compensated for within the early postoperative period by marked paradoxical septal motion. RV end systolic dimensions rose by $41 \%(2.65(0.33)$ to $3.39(0.42) \mathrm{cm})$ and end diastolic dimensions by $29 \%(3.08$ $(0.37)$ to $4 \cdot 28(0.87) \mathrm{cm})$. Left ventricular function was preserved after surgery. Catheterisation before hospital discharge showed that RV pressure waveforms were markedly abnormal, consistent with the conduit nature of the right ventricle. Postoperatively mean right atrial pressure was $17 \mathrm{~mm} \mathrm{Hg}$. Acute renal impairment occurred in all six patients; it was worst on day 7 (mean urea $21.7 \mathrm{mmol} / 1$, mean creatinine $275 \mu \mathrm{mol} / \mathrm{l}$ ) but normal by day 18 without recourse to dialysis. The mean duration of intensive care treatment was 106 hours (range 24-408) and the mean postoperative hospital stay was 17 days (range 11-35). All patients survived surgery, returned to high functional status without diuretic treatment, and were arrhythmia free at a mean postoperative follow up of 19 months (range 2-65).

Despite the radical nature of this operation and its acute haemodynamic problems, it is effective in arrhythmia suppression and surprisingly well tolerated in the long term.

\section{Effects of adenosine on monophasic action potential in humans}

\section{S O Nunain, C Garratt, V Paul, Nadia Debbas, D Ward, A J Camm \\ St George's Hospital, London}

We assessed the effects of therapeutic doses of adenosine on human atrial and ventricular repolarisation in 15 patients (11 men; aged 18-61) undergoing diagnostic electrophysiological studies. The atrial monophasic action potential (MAP) was recorded continuously during constant rate atrial pacing (cycle length $495.4(70) \mathrm{ms}$ ). The MAP duration to $90 \%$ repolarisation $\left(\mathrm{MAPD}_{90}\right)$ was measured at baseline after intravenous adenosine $6 \mathrm{mg}$ and $12 \mathrm{mg}$. The ventricular MAPD $_{90}$ was measured in five patients during ventricular pacing after $12 \mathrm{mg}$ adenosine. After $6 \mathrm{mg}$ adenosine the atrial $\mathrm{MAPD}_{90}$ shortened from 223.1 $(27.6)$ to $185.6(28.3) \mathrm{ms}(\mathrm{p}<0.005)$. After $12 \mathrm{mg}$ the atrial MAPD $_{90}$ shortened to $168(31 \cdot 1) \mathrm{ms}(\mathrm{p}<0.001)$. After $12 \mathrm{mg}$ the ventricular $\mathrm{MAPD}_{90}$ showed no significant change (247 (28.3) to $242(27 \cdot 3) \mathrm{ms})$. The magnitude of the effect on the atrium between the two doses of adenosine was significant $(p<0.01)$ as was the difference between the effect on the atrium and ventricle $(p<0.005)$. The effect on the atrium began at $10.7(7 \cdot 3) \mathrm{s}$ and reached a maximum at $21.6(7.9) \mathrm{s}$ at the time of atrioventricular block or onset of symptoms.

Adenosine in therapeutic doses markedly shortens atrial but not ventricular MAPD $_{90}$ in a dose dependent manner. This may explain its tendency to provoke atrial fibrillation.

\section{Skin blood flow in women with angina pectoris and normal coronary arteries: effects of oestrogen treatment}

D C Lindsay, G M C Rosano, P M Sarrel, P A Poole-Wilson

National Heart and Lung Institute, London

Many patients with chest pain and angiographically normal coronary arteries (syndrome $\mathrm{X}$ ) are women of perime- 
nopausal age. Syndrome $\mathrm{X}$ has been shown to be associated with peripheral and coronary vasomotor dysfunction. Climacteric symptoms such as hot flushes and migraine are caused by vasomotor instability, and dyspnoea and chest discomfort may also occur perimenopausally. Such symptoms are well controlled by hormone replacement therapy. In this study evidence for peripheral vasomotor dysfunction was sought in 20 women with syndrome $X$ and 10 age matched female controls by measuring the cutaneous hyperaemic response. The finger tip blood flow (expressed in $\mathrm{mV}$ ) was measured by laser Doppler velocimetry after the release of five minutes of arterial occlusion. Ten of the subjects with syndrome $\mathrm{X}$ were treated with two months of transdermal $17 \beta$-oestradiol and then retested in a similar manner. The patients with syndrome $\mathrm{X}$ had a higher baseline blood flow compared with controls before treatment (751 (372) v $318(270) \mathrm{mV}, \mathrm{p}<0.05)$ and a lower maximum to baseline flow ratio after release of the blood pressure cuff $(0.99 v 3.8, \mathrm{p}<0.01)$. After treatment a significantly lower baseline $(295(230)$ v $751(372) \mathrm{mV}, \mathrm{p}<0.002)$ and a significant increase of maximum to baseline ratio $(2.07 v$ $1.06, \mathrm{p}<0.01)$ compared with pretreatment values were found. No significant difference in resting blood flow (295 (230) $v 318(270) \mathrm{mV}, \mathrm{NS}$ ) or ratio of hyperaemic response maximum to baseline $(2 \cdot 07 v 2 \cdot 31$, NS) was found between patients after oestrogen treatment and normal controls.

Thus patients with syndrome $\mathrm{X}$ have a higher baseline flow and a lower maximum hyperaemic to baseline ratio compared with normal subjects. Oestrogen replacement therapy normalises peripheral blood flow in patients with syndrome $\mathrm{X}$, and the potential beneficial effects on coronary flow and symptoms need to be evaluted.

\section{Acute vasodilator response of pulmonary vascular} bed in primary pulmonary hypertension

\section{N G Uren, P F Ludman, T Crake, Celia M Oakley Clinical Cardiology Department, Hammersmith Hospital, London}

To study the acute vasodilator response of the pulmonary vascular bed in primary pulmonary hypertension five patients (four women, mean age 34 years, range 21-45) were studied. Haemodynamic parameters were recorded at baseline (B) and after incrementally increasing intrapulmonary infusions of acetylcholine at $3.4 \times 10^{-13}$ to $3.4 \times 10^{-8} \mathrm{~mol} / \mathrm{min}$ and calcitonin gene related peptide (CGRP) at 0.5 to $2 \times 10^{-11} \mathrm{~mol} / \mathrm{min}$ at five minute intervals or until a reduction in systolic systemic artery pressure of $25 \mathrm{~mm} \mathrm{Hg}$ occurred. Oral nicardipine was then given at 60 minute intervals (dose range $100-200 \mathrm{mg}$ ). There was no significant increase in stroke volume from baseline with acetylcholine or CGRP (42 (SD 19) at baseline to 46 (23) $\mathrm{ml}$ with acetylcholine; $46(23)$ at baseline to $50(28) \mathrm{ml}$ with CGRP), but nicardipine increased stroke volume from $34(15)$ to $50(19) \mathrm{ml}(\mathrm{p}<0.05)$. There was no significant reduction in systolic pulmonary artery pressure with acetylcholine, CGRP, or nicardipine. Total pulmonary vascular resistance was reduced with nicardipine from $7 \cdot 4$ (4.9) to 4.3 (3.1) units $(\mathrm{p}<0.05)$ but not with acetylcholine or CGRP. Systemic artery pressure fell from 132 (34) to $113(36) \mathrm{mm} \mathrm{Hg}(\mathrm{p}<0.05)$ with CGRP and from 134 (32) to $99(26) \mathrm{mm} \mathrm{Hg}(\mathrm{p}<0.01)$ with nicardipine.

Thus, despite a reversible component to the increased total pulmonary vascular resistance in primary pulmonary hypertension, infusion of acetylcholine and CGRP do not significantly reduce systemic pulmonary artery pressure or total pulmonary vascular resistance. This may be due to a defect in endothelial cell function.

\section{New clinical index of left ventricular mechanical performance}

\author{
J J Stewart, I A Simpson, H H Gray, R E Smith, \\ C Callicott, A J Camm
}

St George's Hospital Medical School, London

The conversion of energy to heat by the left ventricle (LV) myocardium is irreversible and represents inefficiency in the transformation of metabolic energy into external work. We have developed a method for the clinical measurement of LV heat production and applied it in 46 patients with chest pain ( 31 men, aged 37 to 72 (mean 57) years) undergoing cardiac catheterisation. Coronary artery disease was documented in 38; eight had normal coronary arteries. LV mechanical efficiency was calculated from measured LV heat production and LV energy supply (calculated from LV oxygen extraction). There was a close linear relationship between LV heat production and LV energy uptake $(\mathrm{r}=0.92, \mathrm{p}<0.0005)$. At rest, under basal conditions, $\mathrm{LV}$ heat production for the whole group was 3.0 (1.4) W and mechanical efficiency was $33 \cdot 1 \%(11 \cdot 7 \%)$. LV mechanical efficiency in the normal group was $44.2 \%(9.7 \%)$ compared with $30.7 \%(10.7 \%)$ in patients with coronary disease $(p=0.004)$. In 27 patients atrial pacing was undertaken to alter LV energetics. LV energy supply increased in these patients from $4.6(2.7) \mathrm{W}$ to $5.9(3.3) \mathrm{W}$ $(\mathrm{p}<0.0005)$, with a similar increase in $\mathrm{LV}$ heat production from $3.0(1.6) W$ to $4.6(2.4) W(p<0.0005)$. As most of the increased energy utilisation appeared as heat rather than work, there was a significant fall in LV mechanical efficiency with pacing from $33.9 \%(13.5 \%)$ to $18.9 \%$ $(15.2 \%)(p<0.0005)$.

These results demonstrate that measurement of $L V$ heat production is a practical means of characterising $\mathrm{LV}$ mechanical performance in the clinical setting.

Identification of patients at risk of late postinfarct ventricular arrhythmias: ventricular late potentials, Holter monitoring, and ejection fraction

E W Chew, P Morton, J G Murtagh, M E Scott, D B O'Keeffe

Cardiac Unit, Belfast City Hospital, Belfast

The two main aims of this study were to establish the prognostic values of ventricular late potentials (VLP), Holter monitoring, and ejection fraction (EF) in identifying patients at risk of late postinfarct ventricular arrhythmias and to determine which filter frequency $(25 \mathrm{~Hz}$ or $40 \mathrm{~Hz})$ provides the better predictor of outcome. Signal averaged electrocardiograms for VLP, measurement of EF, and Holter monitoring were performed in 211 consecutive patients with first infarcts before discharge. Over the maximum follow up period of 20 months (mean 10.8) there were nine sudden deaths and two patients presented with ventricular tachycardia. Of the 11 who had arrhythmic events (group 1), all 11 had VLP $(40 \mathrm{~Hz})$ compared with 45 $(22.5 \%)$ of those without arrhythmic events (group 2). The figures with the $25 \mathrm{~Hz}$ filter were $2(18.2 \%)$ and $15(7.5 \%)$ respectively. Eight $(72 \cdot 7 \%)$ patients in group 1 and 45 $(22.5 \%)$ in group 2 had couplets or salvos on Holter monitoring. Five $(45.5 \%)$ patients in group 1 and $20(10 \%)$ in group 2 had EF less than $40 \%$. VLP $(40 \mathrm{~Hz})$ predicted arrhythmic events with a sensitivity of $100 \%$ and a specificity of $77 \cdot 5 \%$. The corresponding values for the $25 \mathrm{~Hz}$ filter were $18.2 \%$ and $92.5 \%$; for Holter monitoring 
they were $72 \cdot 7 \%$ and $77 \cdot 5 \%$ and for EF $45.5 \%$ and $90 \%$. Multifactor analysis identified VLP $(40 \mathrm{~Hz})$ and Holter monitoring as the only independent predictors of outcome. Their combination had a sensitivity of $72.7 \%$ and a specificity of $92.5 \%$.

In conclusion, both VLP and Holter monitoring are important predictors of arrhythmic events and the $40 \mathrm{~Hz}$ filter provides the better predictor of outcome.

\section{Circadian variation in white blood cell aggregation and free radical indices in healthy volunteers}

\author{
A B Bridges, T C Fisher, M McLaren, N A Scott, \\ J J F Belch \\ Departments of Cardiology and Medicine, Ninewells \\ Hospital, Dundee
}

Previous studies have shown circadian rhythms in the onset of thrombotic events, which occur most commonly in the morning, and also in the fibrinolytic activity of the blood, which has a peak in the evening and a trough in the morning. There has recently been increasing interest in the role of white cells and free radicals in thrombosis. No one has yet investigated the potential circadian variation of white cell aggregation and free radicals in humans. We studied the circadian rhythm of white cell aggregation and free radical status in 10 healthy male volunteers. Six blood samples were collected at four hourly intervals from 12 midday until $8 \mathrm{am}$ the following morning. The volunteers carried out normal daily activities until 12 midnight, when they went to bed, where they remained until $8 \mathrm{am}$. The following were measured in each sample: white cell aggregation; malonyldialdehyde concentration, which is a product of lipid peroxidation by free radicals; free radical scavengers; plasma thiols ( $\mathrm{PSH}$ ); red cell lysate thiols (LSH); glutathione (GSH) and superoxide dismutase (SOD) activities, which are all altered in the presence of free radical activity. The following parameters showed significant circadian rhythms: white cell aggregation $(p<0.001)$, malonyldialdehyde $(p<0.015)$, PSH $(p<0.001)$, LSH $(p<0.002)$. White cell aggregation was lowest at $9 \mathrm{am}$ and highest at midnight to $4 \mathrm{am}$. Malonyldialdehyde and PSH both had a peak at $4 \mathrm{pm}$ and a trough at $4 \mathrm{am}$. LSH had a peak at 12 noon and a trough at 8 am.

As the behaviour of white cells and free radical state influence the flow properties of blood, a circadian rhythm in white cell function and free radical state may contribute to the time of onset of thrombotic diseases. Moreover, with many studies being currently undertaken in this subject, our work indicates the need to standardise sample times.

\section{Effects of captopril and of isosorbide mononitrate on ventricular arrhythmias during acute phase of myocardial infarction: randomised trial}

\section{A Pipilis, M Flather, R Collins, Clare Foster, P Sleight} Cardiac Department, John Radcliffe Hospital, Oxford

To assess the effects of oral vasodilator treatment on ventricular arrhythmias during the early phase of myocardial infarction 76 patients (mean age 61 (9) years) were randomised at a mean of 12 hours from the onset of pain to captopril $(12.5 \mathrm{mg}$ three times a day after a $6.25 \mathrm{mg}$ test dose; $\mathrm{n}=27$ ) or isosorbide mononitrate (ISMN; $20 \mathrm{mg}$ three times a day; $n=23)$ or placebo $(n=26)$. The clinical characteristics of the three groups were closely matched and $95 \%$ of the patients received thrombolysis. All patients had 48 hours of Holter monitoring starting at the time of randomisation. For each patient the number of ventricular ectopics (VE) per hour, multiplets per hour, and episodes of ventricular tachycardia (VT) were calculated for the whole 48 hour period. VE and multiplets per hour were logarithmically transformed to obtain normal distributions. The mean log VE per hour for the placebo, captopril, and ISMN groups were respectively $1 \cdot 13$ (SEM $0 \cdot 18$ ), $0 \cdot 74$ $(0.14)$, and $0.49(0.12)(\mathrm{p}=0.005$ for ISMN $v$ placebo, all other comparisons NS). A similar trend was seen for log multiplets per hour: $0.03(0.16),-0.39(0.14)$, and -0.59 $(0.09)(p=0.005$ for ISMN $v$ placebo). The frequency of episodes of VT in the active treatment groups was not significantly less than that in the placebo group: $5.5(1.8)$ for placebo, 3.0 (0.9) for captopril, and $2 \cdot 1(0 \cdot 7)$ for ISMN. During the first six hours after randomisation $42 \%$ of the placebo, $15 \%$ of the captopril, and $9 \%$ of the ISMN groups had at least one episode of idioventricular rhythm ( $\mathrm{p}<0.05$ for captopril $v$ placebo and $\mathrm{p}<0.01$ for ISMN $v$ placebo).

In summary, these data suggest that ISMN and perhaps to a lesser degree captopril reduce the incidence of ventricular arrhythmias in the acute phase of myocardial infarction. Further study on far larger numbers is required to determine whether these effects are translated into reductions in serious arrhythmias, such as ventricular fibrillation, and death.

\section{Autonomic nerve dysfunction in patients with silent myocardial ischaemia}

\section{F Shakespeare, A Crowther, I C Cooper,} M M Webb-Peploe

Department of Cardiology, St Thomas's Hospital, London

Anginal pain is thought to be mediated via cardiac sympathetic afferent nerves. The aim of this study was to examine whether patients with silent myocardial ischaemia have evidence of autonomic dysfunction. Forty two male patients with angiographic evidence of coronary artery disease and demonstrable ischaemia on 24 hour Holter monitoring with a Reynolds solid state recorder were selected. Patients were assigned to group $1(n=21)$ if they had only silent ischaemic episodes and to group $2(n=21)$ if there were any symptomatic ischaemic episodes. Both groups underwent autonomic testing using the Valsalva manoeuvre, isometric hand grip, lower body negative pressure, and cold pressor tests. Measurements of heart rate; plethysmographic forearm blood flow (FBF) and vascular resistance (FVR); and systolic, diastolic, and mean arterial pressure were made during the latter three tests. In both groups there was no significant difference in mean age or presence of systemic hypertension. During the isometric hand grip there was a significant difference between the groups in the increase in FBF $(83.2 \%$ in group $1,48.5 \%$ in group $2 ; \mathrm{p}<0.03)$ and reduction in FVR (35\% in group 1, $20.4 \%$ in group $2 ; p=0.02$ ). Mean arterial pressure rose by a mean of $10 \mathrm{~mm} \mathrm{Hg}$ in both groups. Between the first and second minute of grip, when FBF is expected to fall and FVR increase, FBF and FVR in group 1 remained unchanged but in group $2 \mathrm{FBF}$ increased by $13.5 \%$ $(p<0.02)$ and FVR fell by $10.4 \%(p<0.01)$. The Valsalva $R R$ interval ratios and lower body negative pressure test 
parameters did not differ between the two groups, suggesting no difference in parasympathetic function. There was no difference between the two groups in the parameters measured during the cold pressor test.

These data suggest that there are differences in autonomic function in patients with pure silent ischaemia compared with those with symptomatic ischaemia.

\section{Thrombolytic treatment for acute infarction: more good news}

J P Bourke, A A Young, D A B Richards, J B Uther Academic Cardiology, Freeman Hospital, Newcastle upon Tyne, and Cardiology Unit, Westmead Hospital, Sydney, Australia

The aim of this study was to determine whether treatment with intravenous streptokinase with or without oral aspirin during infarct evolution reduces the incidence of inducible ventricular tachycardia at electrophysiological study. Of 159 patients randomised to the multicentre, placebo controlled ISIS-2 study of streptokinase and aspirin in myocardial infarction, 87 underwent electrophysiological study 6-28 days later for prognostic reasons to determine their risk of late spontaneous ventricular tachycardia. Randomisation resulted in four clinically similar patient groups (streptokinase 20; aspirin 25; streptokinase and aspirin 21; both placebo 21 ). With an electrophysiological study protocol with $\leqslant 4$ extrastimuli only the induction of ventricular tachycardia of cycle length $\geqslant 230 \mathrm{~ms}$ for $\geqslant 10 \mathrm{~s}$ was considered abnormal. Antiarrhythmic drug treatment was not routinely prescribed regardless of the result of electrophysiological study. Ventricular tachycardia was induced in eight out of 46 patients treated with placebo streptokinase $v$ none out of 41 treated with active streptokinase $(\mathrm{p}<0.005)$ and in four out of 41 patients treated with placebo aspirin $v$ four out of 46 treated with active aspirin (NS). During mean follow up of 39 (9) months no patient treated with active streptokinase had spontaneous ventricular tachycardia compared with three treated with placebo streptokinase $(\mathrm{p}=0 \cdot 13)$.

Streptokinase treatment during acute myocardial infarction markedly reduces the incidence of inducible ventricular tachycardia in infarct survivors. Aspirin has no such effect.

\section{White cell aggregation in coronary disease}

Ann C Tweddel, W Martin, Jill Belch, D J Wheatley, I Hutton

Department of Medical Cardiology, Royal Infirmary, Glasgow, and Department of Medicine, Ninewells Hospital, Dundee

White cell activation has been implicated in acute myocardial ischaemia, and this may be reflected in increased white cell aggregation. This ability of white cells to aggregate in response to formyl-methionyl-leucylphenylalanine can be compared with a standard, allowing percentage aggregation to be measured in whole blood. In 98 patients with established coronary artery disease and 24 normal subjects there were no significant differences in random samples. (Normals $43.7 \%(10.6 \%)$ and patients $47 \cdot 9 \%(12 \cdot 3 \%)$. Aspirin $(46 \cdot 7(12 \cdot 1), \mathrm{n}=60), \beta$ receptor blockers (47.3 (13.3), $\mathrm{n}=54)$, nitrates (45.8 (12.9), $\mathrm{n}=60$ ), and calcium channel blockers seemed not to affect aggregation (47.1 $(12 \cdot 7), \mathrm{n}=67)$. In 25 patients with angiographically established coronary artery disease, aggregation increased significantly with symptom limited maximal exercise $(53.0(9.6)$ to $58.6(8.3)$ at peak, $p<0.01)$ and had returned towards baseline at 10 minutes after exercise. In patients with chest pain $(n=9)$ as the limiting symptom aggregation increased significantly by $19 \%$ compared with $8 \%$ in patients limited by breathlessness or leg fatigue.

These results suggest white cell aggregation may be induced in response to cardiac ischaemic pain and that this may be relevant in patients with acute ischaemic syndromes.

\section{Cardiac structure and function in postmenopausal women: effects of long term oestrogen replacement therapy}

D J Walsh, J M McLenachan, Esther Henderson, Eileen Lundmark, D McKay-Hart, H J Dargie Department of Cardiology, Western Infirmary, Glasgow

Anxiety concerning possible deleterious cardiovascular effects of long term oestrogen therapy has been replaced by recent reports suggesting a potential cardioprotective role for hormone replacement therapy. There are, however, few studies documenting the long term effects of hormone replacement therapy on cardiac structure and function. As part of a randomised single blind study 49 women who had been taking either oestrogen treatment $(n=26)$ or matching placebo $(n=23)$ for at least 10 years underwent non-invasive cardiovascular assessment including echocardiography and exercise testing. The two groups were matched at baseline for age, arterial pressure, and body mass index. After treatment for 12-21 years bone mineral density was, as expected, significantly higher in the oestrogen treated group than in the controls $(114.2(3.5) v$ $88.9(2.9), \mathrm{p}<0.0001)$. On echocardiography left ventricular end systolic dimension was significantly lower in the active treatment group than in the placebo group $(26.2(0.9) v 29.9(1.5) \mathrm{mm}, \mathrm{p}<0.05)$ and fractional shortening significantly greater $(37.6 \%(1.8 \%) v 31 \cdot 7 \%(2.3 \%)$, $\mathrm{p}<0.05)$. Left ventricular mass was slightly, but not significantly, lower in the oestrogen treated group (195(9) $v 213(15) \mathrm{g}$, NS). Blood pressures were similar in the two groups. During treadmill exercise testing there were no differences between the two groups in terms of exercise time, development of chest pain, and maximum ST segment depression.

Thus long term oestrogen treatment does not seem to be associated with adverse cardiovascular adaptations and may have beneficial effects on cardiac structure and function.

Hyperinsulinaemic response in chronic heart failure is significantly improved by captopril

R A Wright, A D Flapan, K G M M Alberti, R A Riemersma, K A A Fox

University of Edinburgh, Edinburgh, and University of Newcastle upon Tyne, Newcastle upon Tyne

We have shown that patients with chronic heart failure show a markedly hyperinsulinaemic response to a $75 \mathrm{~g}$ oral 
glucose tolerance test. Captopril has been shown to improve insulin sensitivity in patients with hypertension. We present the first report of the effect of angiotensin converting enzyme inhibition with captopril on hyperinsulinaemia in chronic heart failure. Twenty three nondiabetic patients ( 18 men, mean age 60 years) with chronic heart failure (New York Heart Association class III) were studied. At baseline all were taking frusemide $(40 \mathrm{mg}$, $\mathrm{n}=12 ; 80 \mathrm{mg}, \mathrm{n}=11$ ) and the dose was not changed. Mean ejection fraction was $20 \%$ (range $10-36 \%$ ) and mean body mass index $25 \cdot 5$ (SEM $0 \cdot 7) \mathrm{kg} / \mathrm{m}^{2}$. A $75 \mathrm{~g}$ oral glucose tolerance test was performed before and four weeks after the introduction of captopril $75 \mathrm{mg}$ daily. Blood samples were taken at $0,30,60,90$, and 120 minutes for measurement of plasma concentrations of glucose, insulin, and non-esterified fatty acids (NEFA). Fasting values of plasma glucose, insulin, and NEFA were unchanged between baseline and after four weeks of treatment with captopril. Plasma insulin concentration fell from $94(11) \mathrm{mU} / 1120$ minutes after glucose in the baseline study to $67(8) \mathrm{mU} / 1$ after the introduction of captopril $(\mathrm{p}<0.01)$. There was no significant change in plasma concentrations of glucose $(7 \cdot 4(0.7) v 6.9(0.45) \mathrm{mmol} / \mathrm{l})$ or NEFA (62 (12) $v 91(34) \mu \mathrm{mol} / \mathrm{l})$ at 120 minutes.

Captopril significantly decreases the late hyperinsulinaemic response to an oral glucose load in patients with chronic heart failure. This effect of captopril is not mediated by a reduction in circulating concentrations of NEFA. Hyperinsulinaemia is associated with sodium retention and impaired muscle metabolism and a reduction in insulin concentrations may correspond to an improvement in clinical wellbeing.

\section{Controlled trial of magnesium supplementation in congestive heart failure: effects on electrolytes, arrhythmias, haemodynamics, and sympathetic activity}

\section{Y Bashir, J F Sneddon, Ann Staunton, W J McKenna, D E Ward, A J Camm \\ St George's Hospital Medical School, London}

Magnesium depletion is often present in congestive heart failure and may exacerbate hypokalaemia, arrhythmias, peripheral vasoconstriction, and neurosympathetic activation. The effects of magnesium supplementation were investigated by a randomised double blind crossover trial in 12 patients with chronic congestive heart failure (New York Heart Association classes II-IV) and frequent $(>500 / 24 \mathrm{~h})$ ventricular ectopic activity who were all taking long term loop diuretic treatment. Subjects alternately received enteric coated magnesium chloride $\left(\mathrm{MgCl}_{2}\right)$ (magnesium $17 \mathrm{mmol} /$ day) or placebo for six weeks. Treatment was well tolerated, but one patient withdrew because of dyspeptic symptoms. Compared with placebo, $\mathrm{MgCl}_{2}$ increased urinary magnesium excretion from $2.93(1.26)$ to $4.77(2.01) \mathrm{mmol} / 24 \mathrm{~h}(\mathrm{p}<0.01)$, but there was no significant rise in serum concentrations of magnesium $(0.88(0.08) \quad v 0.92(0.06) \mathrm{mmol} ; \mathrm{NS})$ or potassium $(3.9(0.4) v 4 \cdot 1(0 \cdot 2) \mathrm{mmol} ; \mathrm{NS}) . \mathrm{MgCl}_{2}$ produced significant suppression of asymptomatic ventricular arrhythmias: mean (range) ventricular ectopic frequency was reduced from $4537(654-14573)$ to 3630 $(184-10964) / 24 \mathrm{~h}(\mathrm{p}=0.05)$, couplets from $261(5-1572)$ to $160(1-1038) / 24 \mathrm{~h}(\mathrm{p}<0.02)$, and episodes of ventricular tachycardia from $57(0-378)$ to $17(0-121) / 24 \mathrm{~h}(\mathrm{p}<0.05)$. There was no difference between placebo or $\mathrm{MgCl}_{2}$ with regard to mean arterial pressure $(91(10) v 88(9) \mathrm{mm} \mathrm{Hg}$;
NS), cardiac index $\left(2 \cdot 31(0 \cdot 47) v 2 \cdot 20(0 \cdot 46) 1 / \mathrm{min} / \mathrm{m}^{2} ; \mathrm{NS}\right)$ and systemic vascular resistance $(1635(541)$ v 1656 (510) dynes $\times \mathrm{s} / \mathrm{cm}^{5} ; \mathrm{NS}$ ), and there was no change in plasma concentrations of noradrenaline $(546(136) v 592$ (220) $\mathrm{pg} / \mathrm{ml} ; \mathrm{NS}$ ) and adrenaline (605(732) v 203 (113) $\mathrm{pg} / \mathrm{ml}$; NS).

In conclusion, magnesium supplementation resulted in suppression of ventricular ectopic activity, particularly repetitive forms, but did not improve haemodynamics or neurosympathetic indices.

\section{Aortic balloon pumping in cardiogenic shock: effects on cardiac output, renal blood flow, and renal oxygen consumption}

G A Haywood, J T Stewart, P J Counihan, J F Sneddon, D Tighe, E D Bennett, J R Pepper, W J McKenna Cardiac Department, St George's Hospital, London

The effect of aortic balloon pumping on renal perfusion during cardiogenic shock has not been reported in humans. We have developed and validated a portable continuous thermodilution method for direct measurement of renal blood flow that allows rapidly repeatable measurements to be taken. In vitro blood flow was measured against known flow rates in a cardiopulmonary bypass circuit with excellent correlation $(R=0.99)$ over the full range of renal blood flows normally encountered in humans (95-1024 $\mathrm{ml} / \mathrm{min}$, mean difference $39(64) \mathrm{ml} / \mathrm{min}$, maximum error observed $13 \%$ ). In vivo left renal blood flow was measured serially in nine pigs by simultaneous renal vein thermodilution and renal artery electromagnetic flow probe before and after induction of shock (difference between methods $23(40) \mathrm{ml} / \mathrm{min}, \mathrm{R}=0.92)$. Cardiac output, mean arterial blood pressure, left renal blood flow, and left renal oxygen uptake were measured in seven patients in cardiogenic shock (cardiac index $<2.5$, mean $1 \cdot 7(0.4)$ ) with aortic balloon pumps in situ. Cardiac output was $3 \cdot 4(0.8) 1 / \mathrm{min}$ during $1: 1$ aortic balloon pumping and $3.0(0.9) 1 / \mathrm{min}$ measured after the balloon had been switched to standby for 15 minutes $(p<0.02)$. However, there was no significant difference in mean arterial blood pressure $(70(6) \mathrm{mm} \mathrm{Hg}$ in $1: 1 v 69(13)$ in standby), left renal blood flow $(210(124) \mathrm{ml} / \mathrm{min}$ in $1: 1 v 197(127)$ in standby), or left renal oxygen uptake $(5.0(3) \mathrm{ml} / \mathrm{min}$ in $1: 1 v 5.0(2)$ in standby).

The renal vein continuous thermodilution technique permits rapid, direct measurement of renal blood flow in patients with cardiogenic shock and is of potential value in guiding treatment directed at increasing renal perfusion. Despite achieving a small but significant increase in cardiac output aortic balloon pumping did not increase renal perfusion or oxygen consumption in this group of patients with renal hypoperfusion and low cardiac output.

\section{Detection of myocardial perfusion by intravenous} contrast digital radiofrequency echocardiography

M J Monaghan, Jennie Metcalfe, S O Odunlami, S D Thomas, A Waaler, D E Jewitt

King's College Hospital, London, and Nycomed AS, Oslo, Norway

Conventional contrast echocardiography techniques have proved inadequate for the detection of myocardial perfusion with the new intravenously injected echo- 
cardiography contrast agents because of the limitations and relative insensitivity of standard echocardiographic equipment. To avoid these problems we have used a modified phased array ultrasound scanner and an on line computer system to obtain pure digital radiofrequency ultrasound (DRF) data (derived from the transducer preamplifiers) from the left ventricle and myocardium during intravenous contrast myocardial perfusion echocardiographic studies. In 10 patients after coronary arteriography intravenous injections of the echocardiography contrast agent Albunex (sonicated human serum albumin) in two doses of 0.08 and $0.22 \mathrm{ml} / \mathrm{kg}$ were given during DRF echocardiography. The DRF data were successfully obtained and analysed in seven patients for a shift in the frequency spectrum which could be caused by resonance of the Albunex contrast microbubbles in the heart-a phenomenon that has been previously shown in vitro. The mean integrated backscatter (MIB, a measure of the total ultrasound energy) was also calculated before, during, and after Albunex injection. In segments where perfusion was predicted to be normal there was a significant increase in MIB from 0.89 to 1.93 units $(p<0.02)$ and time intensity curves could be constructed showing wash in and out of contrast from the myocardium. Peak contrast intensity in the myocardium was delayed by a mean of six beats from peak left ventricular intensity. In four segments where very poor perfusion was predicted MIB intensity increased by only $7 \%$ and wash out was prolonged. In all myocardial segments contrast microbubble resonance also caused a drop in mean frequency of $140 \mathrm{kHz} \quad(\mathrm{p}<0.005)$ and permitted similar temporal analysis of myocardial perfusion. Frequency changes preceded intensity changes in both the left ventricle and myocardium by $2 \cdot 3$ beats and represent faster pulmonary transmit of smaller contrast microbubbles.

In conclusion, this study shows for the first time that DRF echocardiographic analysis provides a method of detecting myocardial perfusion after intravenous Albunex.

\section{Coronary artery ultrasonography with $7 \cdot 5 \mathrm{MHz}$ transducer: comparison with histopathology}

Antoinette Kenny, Catherine Fuller, N R B Cary, L M Shapiro

Papworth Hospital, Cambridge

High frequency epicardial echocardiography (HFEE) is a technique that directly images the coronary artery by epicardial placement of an ultrasound transducer. It may therefore provide information intraoperatively supplementary to coronary angiography. The purpose of this study was to assess the accuracy and reproducibility of the technique in locating coronary arteries, measuring dimensions, and identifying atherosclerotic lesions. Five pressure perfused postmortem human hearts were studied with a 7.5 $\mathrm{MHz}$ Vingmed/Sonotron transducer and comparison made with histopathological examination. Twenty two coronary arteries were imaged, luminal diameter range $0.9-0.5 \mathrm{~mm}$. There was close agreement between ultrasound and pathological measurements of luminal diameter $(\mathrm{r}=0.99, \mathrm{p}<0.0001, \mathrm{n}=26)$. Intraobserver and interobserver variability measurements showed excellent reproducibility, the coefficient of variation being $3.95 \%$ and $3.8 \%$ respectively. The resolution of the transducer was not high enough to measure wall dimensions accurately but could readily identify diffuse wall thickening and calcification. HFEE was accurate in identifying atherosclerotic lesions, imaging arteries distal to an occlusion, locating deeply sited arteries, and identifying complete obliteration of an artery.

HFEE may therefore provide valuable information intraoperatively. Cardiac movement and restricted access may pose difficulty to the in vivo use of HFEE. However, with video playback facilities and transducer miniaturisation the intraoperative assessment of coronary artery disease may become a real possibility.

\section{Quantitation of spatial velocity characteristics in hypertrophic cardiomyopathy: on line digital analysis of colour Doppler flow map images}

\section{A Simpson, J F Sneddon, Gill Smith, A J Camm, W J McKenna \\ Department of Cardiology, St George's Hospital, London}

Digital computer analysis of colour Doppler flow mapping can provide quantitative spatial velocity information. We studied 14 patients with hypertrophic cardiomyopathy by using a Vingmed CFM750 with a $3.5 \mathrm{MHz}$ transducer. Images were obtained in the velocity variance mode with low pulse repetition frequency and moderately high gain. On line digital data transfer was performed to an Apple IICi computer, with maximum outflow tract velocity estimated from the spatial change in velocity on colour flow mapping. The computer digitised data were then subjected to multiple alias unwrapping, yielding velocities ranging from $0.94-5.6 \mathrm{~m} / \mathrm{s}$ which correlated well with continuous wave Doppler velocities $(0.98-5.7 \mathrm{~m} / \mathrm{s}, \mathrm{r}=0.95)$. The site of maximum velocity on colour flow mapping occurred at the point of maximum systolic anterior mitral valve motion in all patients. In five patients with significant systolic obstruction high levels of variance occurred in the subaortic region distal to anterior mitral motion (average pixel variance $35 \% v 2 \%$ in patients with no obstruction). One patient with a subaortic muscle shelf but no obstruction had a very small associated area of high variance but a normal averaged outflow tract variance value. Spatial acceleration (in $\mathrm{cm} / \mathrm{s}$ per $\mathrm{cm}$ ) proximal to the site of $\max$ imum obstruction was quantified from the spatial change in velocity. The maximum rate of spatial acceleration occurred just proximal to systolic mitral motion and in the five patients with obstruction was $>240 \mathrm{~cm} / \mathrm{s}$ per cm compared with $<70 \mathrm{~cm} / \mathrm{s}$ per $\mathrm{cm}$ in the nine patients with no obstruction.

Digital data analysis of colour flow map images can quantitate velocities, spatial acceleration, and the presence and extent of disturbed flow in hypertrophic cardiomyopathy and can provide important functional insights into the flow dynamics and obstructive characteristics in such patients.

\section{Myocardial infarction with complete heart block treated with thrombolysis}

M J D Roberts, J A Purvis, A J McNeill, T G Trouton, G W N Dalzell, G C Patterson, N P S Campbell, $M$ M Khan, S W Webb, A A J Adgey

Regional Medical Cardiology Centre, Royal Victoria Hospital, Belfast

Acute myocardial infarction is complicated by complete heart block in $7-11 \%$ of cases, most often complicating 
inferior myocardial infarction. Thrombolytic treatment has frequently been withheld from patients with complete heart block after myocardial infarction because of the presence of significant hypotension and the risk of bleeding should temporary pacing be required. Nevertheless, successful coronary thrombolysis results in improvement in perfusion of the conduction system. We treated 21 patients (12 male, nine female) with thrombolysis for acute myocardial infarction (20 inferior myocardial infarction) complicated initially by complete heart block; two patients had had a previous myocardial infarction. Thrombolytic treatment was started at a mean of 154 minutes after the onset of symptoms, and 12 patients received prehospital thrombolysis. All patients had previousty received intravenous atropine. Of the 12 who remained in complete heart block at the time of receiving the thrombolytic agent, 10 reverted to $1: 1$ atrioventricular conduction and one to chronic atrial fibrillation within two hours of treatment. Only four patients required temporary transvenous pacing. Coronary artery patency during the hospital stay was $15 / 19(79 \%)$ and the mean global left ventricular ejection fraction was $57 \%(10 \%)$ by radionuclide ventriculography. No haemorrhagic complications occurred. Ventricular fibrillation occurred in four patients during the first 24 hours after myocardial infarction. Late ventricular fibrillation occurred in one patient during reinfarction 15. days after thrombolytic treatment. Only one patient with an anterior myocardial infarction and cardiogenic shock died.

Thus thrombolysis for myocardial infarction complicated by complete heart block is a safe procedure that frequently results in resumption of normal conduction, reducing the need for pacing and improving prognosis.

\section{Study of force interval relations in atrial fibrillation in humans}

\section{S M C Hardman \\ Charing Cross Hospital, London}

Beat to beat variations in the pulse during atrial fibrillation have conventionally been attributed to time dependent changes in filling. I explored the alternative possibility that they are dependent on the intrinsic myocardial interval force relation. Left ventricular contractility (peak rate of rise of pressure $\left(\mathrm{LVdP} / \mathrm{dt}_{\max }\right)$ ) and the aortic velocity integral, which is related to stroke volume, were measured during cardiac catheterisation in 12 patients with atrial fibrillation. Within the range of intervals that determine the interval force relation a positive relation was found between preceding interval, measured from the electrocardiogram (ECG), and contractility-mechanical restitution, and an inverse relation was found between pre-preceding interval and contractility-postextrasystolic potentiation. The variations in contractility contributed significantly to the variations in the aortic velocity integral. These relations were confirmed in the patients during pacing protocols which introduced single extrasystoles into steady state pacing. ECG sequences were borrowed from three of the patients with atrial fibrillation to drive isometrically contracting isolated guinea pig papillary muscle and postextrasystolic potentiation was again found. The maximum rate of rise of force in the isolated muscle correlated well with $\mathrm{LVdP} / \mathrm{dt}_{\max }$ in the patients.
I conclude that interval force mechanisms contribute significantly to the characteristic beat to beat variation of the pulse found in atrial fibrillation:

\section{Endocardial modulation of myocardial contraction}

\section{A M Shah}

Department of Cardiology, University of Wales College of Medicine, Cardiff

Recent experiments with-isolated heart muscle preparations have shown that endocardium can influence contraction of underlying myocardium by analogy with the role of vascular endothelium in regulating blood vessel tone. Selective endocardial damage abbreviates the duration of contraction-a novel "inotropic" effect which differs from that of other interventions and carries pathophysiological implications particularly for early diastolic filling. This study addresses predominantly the mechanisms underlying the influence of endocardium on myocardial contraction. Experiments with isolated muscle and a newly developed endocardial cell culture preparation show that endocardial cells release two humoral agents which have opposing effects on myocardial contraction: (a) an unidentified contraction prolonging factor ("endocardin") and (b) a contraction abbreviating factor, which seems to be endothelium derived relaxing factor, is released both basally and in response to specific stimuli, and acts by raising myocardial cyclic guanosine phosphate concentration.

\section{Left ventricular energetics: heat production by human hearts}

\section{J T Stewart \\ St George's Hospital Medical School, London}

Left ventricular heat production represents inefficiency in the transformation of metabolic energy into left ventricular work. Human left ventricular heat production was estimated in 64 patients ( 46 male, 18 female, mean age 54.2 years) from coronary blood flow, coronary arteriovenous temperature difference, and the areas under thermodilution curves recorded in the aorta and coronary sinus after injection of cold saline into the pulmonary artery. Left ventricular mechanical efficiency was derived from heat production and the energy value of myocardial oxygen utilisation. Left ventricular heat production correlated well with substrate utilisation $(r=0.91, p<0.0005)$. Under basal conditions heat production was $3.0(1.5) \mathrm{W}$ and mechanical efficiency was $30.5 \%(11.5 \%)$. Left ventricular heat production was higher and mechanical efficiency lower in patients with cardiac diseases and transplanted hearts than in patients with normal hearts. In 32 patients atrial pacing was undertaken to alter left ventricular energetics. Left ventricular energy supply increased from 4.9 $(2.6) \mathrm{W}$ to $6.3(3.3) \mathrm{W}(\mathrm{p}<0.0005)$; heat production increased $(3.3(1.7) \mathrm{W}$ to $5.0(2.5) \mathrm{W}, \mathrm{p}<0.0005)$, but mean external power was not altered. Consequently, left ventricular mechanical efficiency fell from $32 \cdot 8 \%$ (13.1\%) to $17.9 \%(14.5 \%)(p<0.0005)$.

These experiments represent the first time that total energy flux has been assessed in intact human hearts. 
Coronary vasodilator myocardial perfusion scintigraphy: ischaemia or redistribution? A controversy revisited

R M John, P I Taggart, P M I Sutton, D C Costa, P J Ell, R H Swanton

Department of Cardiology, Middlesex Hospital and Institute of Nuclear Medicine, University College and Middlesex School of Medicine, London

Myocardial perfusion scintigraphy with coronary vasodilators is invaluable for the detection of coronary artery disease in patients unable to exercise. Controversy exists about whether the mechanism of differential radiotracer uptake is based on ischaemia or merely redistribution. The infrequent occurrence of electrocardiographic changes supports the latter as the principal mechanism. To appraise the incidence and severity of ischaemia we combined recordings of a more sensitive electrophysiological marker of localised ischaemia - namely, monophasic action potentials (MAPs) - with dipyridamole perfusion scintigraphy. The duration of the MAP shortens in response to ischaemia. After routine coronary angiography single site MAP recordings were obtained from the left or right ventricular endocardium, or both, in 31 patients ( 50 recording sites) during intravenous infusion of dipyridamole $(0.015 \mathrm{mg} / \mathrm{kg} / \mathrm{min}$ for 4 minutes $)$. Heart rate was held constant with atrial pacing at $20 \%$ above the patient's resting rate. Technetium- $99 \mathrm{~m}$ methoxy-isobutyl isonitrile was administered four minutes after dipyridamole and single proton emission computed tomography imaging performed an hour later. Rest images were obtained the following day (two day, two dose protocol). Based on perfusion characteristics and coronary anatomical data for the MAP recording site, recordings were grouped into group 1, areas with a normal perfusion pattern $(n=30)$; group 2, areas with a perfusion defect without angiographic collateralisation $(n=10)$; and group 3 , areas with a perfusion defect with angiographic evidence for collateral supply $(n=10)$. Shortening of the MAP duration was apparent in 18 of the 20 recordings from areas of abnormal perfusion. Mean (SEM) values for alteration in MAP duration between control and peak effect at eight minutes were $276.5(5.3)$ to $274.5(5.4) \mathrm{ms}$ in group 1 (NS), 289.6 $(4.7)$ to $278.4(4.8) \mathrm{ms}$ in group $2(\mathrm{p}<0.002)$, and 269.6 $(5.8)$ to $242(4.4) \mathrm{ms}$ in group 3 (p $<0.0001)$. ST segment shift in the electrocardiogram was evident in only eight of the 29 patients with significant coronary stenoses; these eight patients had collateralised viable areas of myocardium.

Our data povide strong evidence for myocardial ischaemia being an integral part of coronary vasodilator perfusion scintigraphy. Ischaemia is of greater intensity and more likely to be overt when myocardial viability is dependent on collateral circulation. The paucity of electrocardiographic changes may be due to the relative insensitivity of the electrocardiogram to early and localised ischaemia.

\section{Asymmetry of cardiac sympathetic innervation in patients with ventricular tachycardia in "clinically normal" hearts shown by iodine-123 m-iodobenzylguanidine scanning}

\author{
J S Gill, G J Hunter, J Gane, D E Ward, A J Camm \\ Department of Cardiological Sciences, St George's \\ Hospital Medical School, London
}

Autonomic influences seem to be important in the genesis of ventricular tachycardia (VT), and these may be particu- larly important in patients in whom VT is exerciseinduced. $m$-Iodobenzylguanidine (MIBG) is an analogue of noradrenaline which is taken up by sympathetic nerve terminals, allowing their visualisation. This study examines the regional distribution of the myocardial sympathetic nerve supply in patients with VT, in "clinically normal" hearts, and in control subjects with iodine-123 MIBG scanning. Thirty one patients were studied, of whom nine had exercise induced VT and eight VT unrelated to exercise; they were compared with six subjects with atrioventricular re-entrant tachycardia and eight patients with angiographically normal left ventricular function, normal coronary arteries, and thallium scans without evidence of reversible or fixed perfusion deficits. Single proton emission computed tomography $\gamma$ scanning was performed in patients immediately and three hours after intravenous injection of $170 \mathrm{mBq} / \mathrm{m}^{2} \mathrm{MIBG}$. The delayed (three hour) left ventricular MIBG uptake data were processed into bull's eye target plots with the IGE Star system, and symmetry of uptake was examined around the vertical diameter. Patients with VT had a higher proportion of asymmetrical MIBG scans $(9 / 17(53 \%))$ than subjects in the control groups $(0 / 14)(p<0.01)$. The number of patients with asymmetrical scans among those with exercise induced VT did not differ from that among those without exercise induced VT (6/9 $v 3 / 8)$. This suggests that patients with VT may have relative sympathetic denervation of the septal portion or less commonly the lateral wall of the left ventricle, leading to an imbalance of the sympathetic supply to the myocardium and locally imbalanced sympathetic-parasympathetic interactions.

Our results suggest that selective denervation of the human myocardium may be an important mechanism predisposing to VT in clinically normal hearts.

\section{Dobutamine/thallium myocardial perfusion tomography}

\section{J Pennell, S R Underwood, R H Swanton,} J M Walker, P J Ell

Departments of Cardiology and Nuclear Medicine, Middlesex Hospital, London

Many factors may prevent a patient performing adequately during exercise testing. While dipyridamole induced coronary arterial dilatation serves as a good alternative during thallium myocardial perfusion imaging, it is difficult to relate abnormalities to levels of exercise achieved in everyday life. Other problems are its variable duration of action and the side effects of peripheral vasodilatation. We therefore examined the use of dobutamine for the manipulation of myocardial oxygen demand in the assessment of coronary artery disease by thallium myocardial perfusion tomography. We studied 50 patients with angina and abnormal exercise electrocardiograms before coronary arteriography. Dobutamine was infused in increasing doses from $5-20 \mu \mathrm{g} / \mathrm{kg} / \mathrm{min}$ until limited by symptoms. Thallium emission tomograms were acquired immediately after and four hours later. Forty patients had significant coronary artery disease, of whom $39(97 \%)$ had abnormal thallium tomograms. Sensitivity was higher for left anterior descending $(92 \%)$ and right coronary $(91 \%)$ lesions than for left circumflex lesions $(83 \%)$. Ten patients had normal coronary arteries, of whom eight had normal thallium tomograms (specificity $80 \%$ ). Dobutamine increased the systolic blood pressure and the heart rate, with a mean increase of $107 \%$ in the double product at $20 \mu \mathrm{g} / \mathrm{kg} / \mathrm{min}$. 
The tolerated dobutamine dose was significantly correlated with the treadmill exercise time. No important arrhythmias were seen.

Dobutamine is a safe and well tolerated agent for the manipulation of myocardial oxygen demand. It is highly effective in the assessment of myocardial perfusion in patients with coronary artery disease. The relation between the achieved dose and treadmill exercise performance increases its value in the clinical assessment of patients with chest pain.

\section{Is an abnormal exercise ejection fraction response on medical treatment of prognostic significance in coronary artery disease?}

\section{R Lim, Lorraine Dyke, D S Dymond}

Department of Cardiology, St Bartholomew's Hospital, London

To evaluate the role of exercise first pass radionuclide ventriculography (RNV) in the prognostic assessment of medically treated coronary disease 54 patients (mean age 57 ) were studied prospectively after coronary angiography. Ejection fraction (LVEF) was measured by RNV with the Scinticor system without anti-ischaemic treatment at a mean of 5.6 weeks after angiography; 32 patients with abnormal results had further RNV while receiving treatment $4 \cdot 2$ weeks later. At nine month follow up 10 of the 54 patients (19\%, group I) had suffered adverse events (one death, one myocardial infarction, one percutaneous transluminal coronary angioplasty, two coronary artery bypass grafting, five unstable angina) and 44 were event free (group II). There was no difference between groups I and II in age, proportion with previous myocardial infarction or positive exercise ECG, number of diseased vessels, or exercise LVEF whether without $(37 \% v 43 \%)$ or with anti-ischaemic treatment $(43 \% v 43 \%)$. Group I tended to show a greater fall in LVEF from rest to exercise without treatment (mean $8 \% v 3 \%, p=0.05$ ). However, with antiischaemic treatment there was a highly significant difference in mean LVEF response to exercise between groups I and II (fall of $10 \% v$ rise of $3 \%, p=0.0006,95 \%$ confidence interval 19 to 6 ).

These results suggest that a persistent abnormal exercise LVEF response despite medical treatment predicts an adverse short term outcome in medically treated coronary disease and may improve patient selection for prognostic revascularisation.

\section{Comparison of same day and separate day injection protocols for myocardial perfusion single proton emission computed tomography with technetium-99m methoxy-isobutyl isonitrile}

\author{
J J Murphy, D R Whallev, M Frier, M L Wastie, \\ R G Wilcox \\ Department of Medicine, University Hospital, Nottingham
}

Although thallium-201 remains the most common radiotracer for myocardial perfusion scintigraphy, it is not an ideal agent and in recent years interest has focused on technetium-99m labelled compounds, notably ${ }^{99 \mathrm{~m}} \mathrm{Tc}$ methoxy-isobutyl isonitrile (MIBI). Unlike thallium-201, ${ }^{99 \mathrm{~m}}$ Tc MIBI undergoes minimal myocardial redistribution so two injections (rest and exercise) are required, the optimum timing and activities of which have yet to be established. To clarify this problem one of three dose administration protocols for ${ }^{99 \mathrm{~m}} \mathrm{Tc}$ MIBI was randomly allocated to each of 45 consecutive patients undergoing investigation for chest pain. The three regimens were $300 \mathrm{MBq}$ at rest and $500 \mathrm{MBq} 24$ hours later on exercise (scheme A); $200 \mathrm{MBq}$ at rest followed by $1000 \mathrm{MBq}$ one and a half hours later on exercise (scheme B); $200 \mathrm{MBq}$ at rest and $600 \mathrm{MBq}$ one and a half hours later on exercise (scheme C). According to symptoms and the results of exercise testing and coronary angiography, patients were allocated by a single observer to one of two clinical groups: "high probability" or "low probability" of ischaemic heart disease. Images obtained from ${ }^{99 \mathrm{~m}}$ Tc MIBI were reported blind by two experienced observers and Cohen's $\kappa$ statistic was used to measure the agreement between imaging and clinical diagnoses. Only for the separate day protocol was $\kappa$ significantly greater than zero at $0.78(95 \%$ confidence interval 0.55 to 1.01$)$. For scheme $B \kappa$ fell to $0.35(0.12$ to $0.58)$ and for scheme $C$ to $0.36(0.13$ to 0.59$)$. The degree of agreement between clinical and imaging diagnoses was significantly better for the separate day than for the same day protocols $(p<0.003)$ whereas schemes $B$ and $C$ did not differ $(p=0.99)$. This difference was explained by significantly fewer false negatives with scheme A. In patients clinically diagnosed as having a high probability of ischaemia only one out of seven was normal compared with nine out of 15 when $B$ and $C$ were combined.

This study shows that despite the incovenience to patients, exercise and rest images must be performed on separate days if myocardial perfusion scintigraphy is performed with ${ }^{99 \mathrm{~m}} \mathrm{Tc}$ MIBI.

\section{Quantitative indium-111 antimyosin antibody imaging for predicting age of acute myocardial infarction}

R Senior, S Bhattacharya, U Raval, J Leppo, A Lahiri Department of Cardiology, Northwick Park Hospital, Harrow, Middlesex

Myocardial uptake of indium-111 antimyosin antibody may occur in the necrotic site for prolonged periods after a myocardial infarction, resulting in difficulty in distinguishing acute from remote events. The uptake intensity may be related to infarct age and may allow more accurate interpretations of antimyosin images. We therefore studied the heart-lung ratio (measured as the ratio of the maximum counts in the infarcted myocardium to the adjacent lung background) in 90 consecutive patients at various intervals after an acute $Q$ wave infarction. Imaging was performed 24 hours after injection of indium-111 antimyosin. In the infarct group 107 studies were performed on the 90 patients between four hours and 270 days after infarction. The heart-lung ratio was inversely correlated with the age of the infarct, with $47 \%$ of the variance being explained by age $(p<0.001)$. Sequential antimyosin imaging was performed in nine patients. The heart-lung ratios were (mean (SD)) $1.77(0.25)$ for images between four and 15 days, $1.54(0.12)$ for images between 50 and 100 days $(p<0.02)$, and $1.42(0.16)(p<0.05)$ for images between 125 and 270 days, confirming the inverse relation with age. A heart-lung ratio above 1.9 had $93 \%$ specificity and an $82 \%$ sensitivity for acute myocardial necrosis and may be used to distinguish acute from remote infarction. 
Reduced skeletal muscle protein synthesis in chronic heart failure

W L Morrison, A Harley, J N A Gibson, K Smith, M J Rennie

Cardiothoracic Centre, Liverpool, and Department of

Physiology and Anatomy, University of Dundee, Dundee

Effort intolerance, muscle weakness, and muscle wasting commonly occur in advanced heart failure. We have investigated protein synthetic rates of quadriceps muscles in vivo with stable isotope techniques. We studied seven men with left ventricular dysfunction (New York Heart Association class IIB; patients' mean age 58 years (range 46-68) and mean weight $69 \mathrm{~kg}(60-75 \mathrm{~kg})$ ) and compared the results with those from 25 control subjects (mean age 45 years (19-82), mean weight $74 \mathrm{~kg}(53-105))$. L- $\left[1-{ }^{13} \mathrm{C}\right]$ leucine was infused after an overnight fast over a seven hour period to achieve'steady state plasma leucine labelling, after which a percutaneous biopsy specimen was taken from the quadriceps muscle. Muscle protein synthesis $\left(k_{s}\right)$ was calculated by comparing ${ }^{13} \mathrm{C}$. leucine enrichment of muscle with that of the plasma measured by gas chromatography mass spectrometry. Muscle DNA concentration was significantly higher in the patients $(3.76$ $(0.55) \mu \mathrm{g} / \mathrm{mg}$ protein) than the controls (2.04 (0.89), $\mathrm{p}<0.01)$. Mean muscle protein synthesis $\left(\mathrm{k}_{\mathrm{s}}\right)$ was 0.033 (SD 0.010$) \% / h$ in the patients compared with 0.045 $(0.015)$ in control subjects $(p<0.01)$. Muscle RNA activity - that is, protein synthetic rate per RNA, which gives an index of the efficiency of protein synthesis-was $0.08(0.03) \mu \mathrm{g}$ of protein $/ \mathrm{h} / \mu \mathrm{g}$ of RNA in the patients compared with $0.018(0.08)$ in control subjects $(p<0.05)$.

In chronic left ventricular dysfunction, even in the absence of clinically overt muscle wasting, there is a substantial depression $(27 \%)$ of skeletal muscle protein synthesis.

Evidence of instability of circulatory regulation in patients with chronic heart failure obtained from ambulatory pulmonary artery pressure recordings

J Simon, R Gibbs, Wendy Sanderson, L D R Smith, Jane Sparrow, K M Fox, P A Poole-Wilson

Royal Brompton National Heart and Lung Hospital, London; National Heart and Lung Institute, London; and IBM Scientific Centre, Winchester

Cardiovascular reflexes that maintain circulatory homoeostasis are abnormal in chronic heart failure. After severe insults to the circulation low frequency vasomotor waves of 0.016 to $0.083 \mathrm{~Hz}$, which represent underdamping of circulatory regulatory mechanisms, have been observed. Ambulatory pulmonary artery pressure measurement with a micromanometer tipped catheter was used to detect such oscillations in heart failure. Spontaneous, sustained low frequency oscillations of pulmonary artery pressure were observed in eight patients (mean age 58 years, range 45 to 67 years) with moderate to severe chronic heart failure (mean maximum rate of oxygen consumption $14.8 \mathrm{ml} / \mathrm{kg} /$ min; mean left ventricular ejection fraction $23 \%$ ) during pulmonary artery pressure recordings of $8.6 \mathrm{~h}$ to $48 \mathrm{~h}$ duration. These oscillations (frequency range 0.015 to $0.035 \mathrm{~Hz}$ ) had a distinct peak form and a lower frequency range than respiration $(0.2$ to $0.6 \mathrm{~Hz})$ and heart rate $(0.8$ to $1.9 \mathrm{~Hz})$. They were present from $42 \%$ to $82 \%$ of the recording time, with an amplitude varying from undetectable to a maximum range from $5.3 \mathrm{~mm} \mathrm{Hg}$ to $19.7 \mathrm{~mm} \mathrm{Hg}$. When detectable, the mean amplitude of the low frequency oscillations of pulmonary artery pressure ranged from 1.2 (SD 1.2) $\mathrm{mm} \mathrm{Hg}$ to $4.3(3.3) \mathrm{mm} \mathrm{Hg}$.

These vasomotor oscillations are evidence for instability of circulatory control in patients with chronic heart failure. At times this control mechanism is markedly underdamped. This finding of circulatory instability may have important implications for the treatment of heart failure which might be aimed at manipulating circulatory control parameters back into the normal range.

\section{Effects of infarct size and physical training on} skeletal muscle metabolism in experimental heart failure in rats

S Adamopoulos, F Brunotte, A J S Coats, J Unitt, D Lindsay, Bheeshma Rajagopalan, G Radda John Radcliffe Hospital, Oxford University, Oxford

The influence of myocardial infarction and physical deconditioning on skeletal muscle metabolism was studied by phosphorus-31 magnetic resonance spectroscopy (MRS) in female Wistar rats 12 weeks after coronary artery ligation $(n=22)$ or sham operation $(n=10)$. Infarcted rats were allocated randomly. to either six weeks training ( $\mathrm{n}=11$; treadmill exercise at $15 \mathrm{~m} / \mathrm{min}$ for $30 \mathrm{~min} /$ day, 6 days/week) or non-training. $(\mathrm{n}=11) .{ }^{31} \mathrm{P}$ spectra were collected from the calf muscle during sciatic nerve stimulation $(1 \mathrm{~Hz}$ ) both supramaximally and submaximally to produce $200 \mathrm{~g}$ tension. Enzymatic assays were performed on the contralateral non-stimulated leg, for glutamate pyruvate transferase (enzyme favouring the removal of pyruvate) and the mitochondrial oxidative enzymes citrate synthase and $\beta$-hydroxyacyl coenzyme A dehydrogenase. Rats were divided into congestive (C) and non-congestive (NC) groups by lung to body weight ratio as an index of severity of heart failure. Changes in creatine kinase activity (CK) were expressed as $\mathrm{CK} / \mathrm{CK}+$ inorganic phosphate and $\mathrm{pH}$ was calculated from the chemical shift of inorganic phosphate $(\mathrm{Pi})$. At $200 \mathrm{~g}$ tension the $\mathrm{C}$ non-trained rats when compared with the trained $\mathrm{C}$ or NC sham operated or NC non-trained rats produced more acid (6.93 (0.03) $v 7.01$ $(0.01), 7.0(0.01), 6.99(0.01), 6.97(0.02), \mathrm{p}<0.05)$ and had lower $\mathrm{CK} / \mathrm{CK}+\mathrm{Pi}(0.49(0.06)$ v $0.67(0.04), 0.71$ $(0.02), 0.71(0.04)$, and $0.72(0.04), \mathrm{p}<0.01$, ANOVA). At maximal tension the $\mathrm{pH}$ in $\mathrm{C}$ non-trained rats was significantly lower than that in all the other groups $(6.86$ $(0.04) v 6.96(0.01), 6.96(0.02), 6.96(0.4)$, and $6.96(0.01)$, $\mathrm{p}<0.01$, ANOVA), while the fall in $\mathrm{CK} / \mathrm{CK}+\mathrm{Pi}$ showed a similar trend to the $200 \mathrm{~g}$ data but was not significant. The C non-trained group had significantly lower citrate synthase activity in skeletal muscle compared with trained Cor $\mathrm{NC}$ and sham groups $(18.3(0.8) v 24.9(0 \cdot 7), 23.5(1.8)$, and $25.9(1.2) \mathrm{U} / \mathrm{gww}$ respectively, p $<0.001$, ANOVA). There was a significant increase in glutamate pyruvate transferase activity in the training compared with nontraining group $(7.9(0.35)$ v $6.5(0.36) \mathrm{U} / \mathrm{gww}, \mathrm{p}<0.03$, ANOVA). There were no differences in $\beta$-hydroxyacyl coenzyme A dehydrogenase activity.

The excessive CK depletion, acidification, and the reduction of mitochondrial enzymes in skeletal muscle of chronic heart failure depend on both physical deconditioning and congestive failure; training may prevent these changes. 
Recovery of impaired endothelium dependent vasodilatation in atherosclerotic coronary arteries

Laura Corr, G Burnstock, P Poole-Wilson Department of Anatomy, University College London and National Heart and Lung Institute, London

Hypercholesterolaemia and atherosclerosis are reported to be associated with impaired endothelium dependent vasodilatation in coronary arteries. We used a model of human familial hyperlipidaemia, the Watanabe hereditable hyperlipidaemic rabbit (WHHL), to examine long term changes in the coronary responses to endogenous vasodilators during the development of atherosclerosis. This genetic model lacks receptors for low density lipoprotein, has a consistently raised plasma cholesterol concentration $(20 \mathrm{mmol} / \mathrm{l})$, and develops early coronary atherosclerosis by 12 months of age. Isolated coronary ring preparations from 22 WHHL aged 4, 6, and 12 months and from 19 age matched New Zealand white controls (NZW) were constricted with $30 \mathrm{mM}$ potassium chloride; the mean tension generated was no different in the two groups. Responses to the endothelium dependent vasodilator substance $\mathbf{P}$ $\left(10^{-7} \mathrm{M}\right)$ were found to be markedly depressed in the four month old WHHL $(2 \cdot 1 \%(1.0 \%))$ compared with the age matched NZW $(48.5 \%(9.9 \%), \mathrm{p}<0.001)$, but there were no significant differences in the relaxant responses to the smooth muscle vasodilators calcitonin gene related peptide (CGRP, $2.6 \times 10 \mathrm{M} ; 74.0 \%$ (3.3) in WHHL v 74.9\% (4.7) in NZW); vasoactive intestinal polypeptide $\left(3 \times 10^{-7} \mathrm{M}\right.$; $52.3 \%(5.9)$ in WHHL $v 60.6 \%(7.1)$ in NZW); and noradrenaline $\left(10^{-4} \mathrm{M} ; 78.2 \%(3.4)\right.$ in WHHL $v 71 \cdot 8 \%(6 \cdot 1)$ in NZW). As the animals matured maximal relaxation to noradrenaline was unaffected while relaxation to CGRP and vasoactive intestinal polypeptide declined in the WHHL; the changes seen in the NZW were very similar. In contrast, relaxation to substance $P$ in the WHHL increased significantly between four and 12 months of age $(p<0.01)$ while in NZW the response to substance $P$ declined significantly over the same period ( $p<0.005)$; by 12 months there was no difference between the two groups. Relaxation to ATP, which acts both directly on the smooth muscle and via the endothelium in this vessel, also declined significantly with age in the NZW $(p<0.05)$ but showed no fall in the WHHL.

These results show that impaired endothelium dependent responses in coronary arteries are capable of long term compensatory recovery during the development of atherosclerosis.

\section{Lack of variation in venous tone in patients with malignant vasovagal syncope}

\section{A D Hargreaves, A L Muir \\ Department of Medicine, Royal Infirmary, Edinburgh}

In the malignant vasovagal syndrome patients seem to develop low ventricular volumes that trigger vagal syncope. In this study we examined the role of the peripheral venous compartment in the sequestration of blood as a cause of abnormal ventricular filling in 31 patients with unexplained syncope. Using a radionuclide blood pool label we measured the percentage change in venous volume on prolonged head up tilting and as an index of venous tone variability or responsiveness, the difference between maximum and minimum calf blood volumes (tilted) being expressed as a percentage of the mean volume. The patients were divided into two groups based on their response to tilt: those who developed syncope or near syncope $(n=6)$ and those who had no symptoms on tilting $(n=25)$. Patients with symptoms had a greater fall in systolic blood pressure $(65.7(10.1) v 14.2(2.4) \mathrm{mm} \mathrm{Hg}$, $\mathrm{p}<0.001)$ and a greater increase in venous volume than those without symptoms $(72 \% \quad(7.4) v 52.0 \% \quad(5.0)$, $\mathrm{p}=0.05)$. However, venous tone variability was significantly less in the patients with symptoms $(4.4 \%(0.9)$ $v 14.1 \%(1.3), \mathrm{p}=0.001)$. The patients with symptoms were older but when compared with patients of a similar age they still had significantly less venous tone variability.

In conclusion, patients with the malignant vasovagal syndrome have an abnormal peripheral venous compartment that does not respond to a falling central pressure, which may cause the progressive sequestration of blood that culminates in vasovagal syncope.

\section{Cardiovascular benefits of closure of arteriovenous fistulas in renal transplant recipients}

J C Doig, A Heads, C Herath, T J H Goodship, $R$ Taylor, $R$ Wilkinson, $S$ Hunter Academic Cardiology, Freeman Hospital, Newcastle upon Tyne

After successful renal transplantation arteriovenous fistulas previously used for haemodialysis are redundant but potentially lead to high output heart failure, thrombosis, and infection. The cardiovascular effects of routine closure of these fistulas have been inadequately explored. Seven patients (four male, three female; mean age 43 years) were studied immediately before and 3 days and 4 months after surgical closure of their arteriovenous fistulas (mean flow $1025 \mathrm{ml} / \mathrm{min}$, range 575-1345). Exercise duration was assessed on Bruce protocol treadmill testing, left ventricular dimensions by echocardiography, and cardiac output by Doppler ultrasonography. Exercise duration increased by $11 \%$ at four months with respect to initial values $(615$ (185) $\mathrm{s}$ to 682 (194) s, $\mathrm{p}=0.012$ ). Maximum systolic blood pressure reached at peak exercise was $7 \%$ lower at four months than preclosure (197 (17) to 183 (18) $\mathrm{mm} \mathrm{Hg}$, $\mathrm{p}=0.016$ ) but the maximum rate-pressure product achieved was unchanged. Resting systolic pressure was lower at four months, having fallen markedly in the acute phase (142 (20) to 131 (19) to $140(11) \mathrm{mm} \mathrm{Hg}$ ). Recovery times were unaltered and there were no ischaemic symptoms or electrocardiographic changes with exercise. Stroke distance fell by $17 \%$ acutely $(29 \cdot 1(5.4)$ to $24.2(6.3) \mathrm{cm}$, $\mathrm{p}=0.019)$ and remained $14 \%$ lower at four months $(24.9$ $(5.5) \mathrm{cm})$. Cardiac output followed this trend $(6.0(2.0)$ to $5.3(1.8)$ to $5.6(1.9) 1 / \mathrm{min})$ but was maintained by a compensatory increase in resting heart rate $(64$ (12) to 70 (14) $(p=0.05)$ to $69(20)$ beats/minute). Left ventricular end systolic dimensions fell by $10 \%$ immediately after closure and remained smaller at four months $(2.63(0.39)$ to 2.36 $(0.38)$ to $2.62(0.69) \mathrm{cm})$. Similarly, end diastolic dimensions fell by $9 \%$ acutely $(4.46(0.44)$ to $4.06(0.42)$ to 4.38 $(0.56) \mathrm{cm})$. Interventricular septal thickness was less at four months than before closure $(18.0(2.3)$ to 17.5 (3.5) $\mathrm{mm}$ ). Drug treatment was constant during the study.

Closure of arteriovenous fistulas in renal transplant recipients whose condition is stable is associated with a number of beneficial cardiovascular benefits and should be routinely performed in transplant recipients. 
Structure and cellular composition of aortic atherosclerotic plaques undergoing ulceration

\section{J Davies, N Woolf, D R Katz}

St George's Hospital Medical School and University College and Middlesex School of Medicine, London

Plaque fissuring is a precursor of acute thrombosis in coronary arteries. The caps of aortic plaques undergo an identical process of tearing leading to ulceration but the time course is more prolonged. Aortic plaques undergoing ulceration were compared with intact plaques of equivalent size in the same aorta. The volume composition of the plaque for lipid and collagen was measured as well as the density of macrophages and smooth muscle cells after identification of the cells by double immunohistochemical staining. Smooth muscle cells were identified by the presence of $\alpha$ actin and macrophages by the presence of the HAM56 antigen. Aortic plaques undergoing ulceration $(n=24)$ when compared with intact plaques $(n=62)$ had a higher proportion by volume of extracellular lipid $(54.9 \%$ (SEM $3 \cdot 8) v 22 \cdot 1 \%(2 \cdot 4))$. The mean macrophage density was increased $(122 \cdot 1(13.3) v 62.2(8 \cdot 8))$ and the mean smooth muscle count was decreased $(65.2(13.2) v$ $122 \cdot 1(13 \cdot 3))$ in ulcerated plaques compared with intact plaques. The ratio of smooth muscle cells to macrophages exceeded 5 in intact plaques but was often less than 1 in ulcerated plaques.

The two major factors predisposing to plaque ulceration are an increase in the volume of extracellular lipid and a decrease in the ratio of smooth muscle cells to monocytes in cap tissue.

\section{Impact of thrombolytic treatment on morbidity and mortality from acute myocardial infarction in clinical practice}

\section{Umachandran, K Ranjadayalan, A D Timmis Newham General Hospital, London}

Thrombolytic treatment reduces infarct mortality in patients selected for randomised trials but its impact on morbidity and mortality in clinical practice has not been clearly defined. We compared the outcome in 178 consecutive patients admitted to our coronary care unit (CCU) during 1990 with 158 consecutive controls admitted during 1986 , before thrombolytic treatment was used in our unit. Only patients fulfilling World Health Organisation criteria for acute infarction were included. Data for 1990 were collected prospectively but, because retrospective controls were used, we were careful to analyse only those end points that could be identified unequivocally from a review of the patient records. Of the 178 admissions in 1990, 154 (87\%) received thrombolytic treatment. This high treatment rate substantially improved morbidity and mortality compared with 1986, presumably because of reductions in infact size. Thus peak creatine kinase activity fell from 2022 (1312) to $1432(1290) \mathrm{IU} / 1(\mathrm{p}<0.001)$ and with it the incidence of cardiogenic shock from $20 \%$ to $6 \%$ ( $p<0.001$ ). Indeed, reductions in all degrees of heart failure were observed, as reflected by take home prescriptions for diuretics, which fell from $42 \%$ to $22 \%$ in patients leaving hospital $(\mathrm{p}<0.001)$. There was also a significant reduction in the incidence of ventricular fibrillation from $22 \%$ to $13 \%$ ( $p<0.05$ ). Hospital mortality in 1986 was $24 \%$ but in 1990 fell to $11 \%(p<0.005)$. It is noteworthy that patients who did not receive thrombolytic treatment in 1990 were a very high risk group with a mortality of $28 \%$ compared with $9 \%$ in the treated group $(\mathrm{p}<0.01)$. Improvements in morbidity and mortality led to reductions in the length of stay in the CCU from $3 \cdot 1(2 \cdot 1)$ to $2 \cdot 0(1 \cdot 4)$ days ( $p<0 \cdot 001$ ).

In conclusion, thrombolytic treatment has substantially reduced hospital mortality in acute myocardial infarction, though not necessarily to the very low levels reported in some clinical trials. It has also had a profound effect on the incidence of major complications, particularly cardiogenic shock and ventricular fibrillation. Additional benefits include reductions in the length of stay in the CCU and the need for long term diuretic treatment, both of which may have important resource implications.

\section{Can thrombolytic treatment produce beneficial effects in the absence of coronary recanalisation during acute myocardial infarction?}

\section{S Chakrabarty, N A Flores, D J Sheridan}

Academic Cardiology Unit, St Mary's Hospital Medical School, London

Evidence suggests that the beneficial effects of thrombolytic treatment cannot be explained by recanalisation of the occluded artery alone and that other factors may be involved. To investigate this we studied changes in haemodynamics, incidence of arrhythmias, and infarct size in anaesthetised rabbits undergoing coronary artery ligation (CAO) with and without intravenous streptokinase $(15000 \mathrm{U} / \mathrm{kg}$ administered 2 minutes after ligation). Reperfusion was initiated by removal of the ligature and infarct size was measured by planimetry of nitro blue tetrazolium stained left ventricular transverse sections $(3 \mathrm{~mm}$ thick). Hearts were studied in four groups: group I $(n=12)$ control hearts with CAO for 60 minutes and no reperfusion; group II $(n=12)$ undergoing CAO for 60 minutes with streptokinase but no reperfusion; group III $(n=12)$ undergoing 30 minutes $C A O$ and 30 minutes reperfusion induced by removal of the ligature; group IV $(\mathrm{n}=12)$ undergoing 30 minutes $\mathrm{CAO}$ with steptokinase and 30 minutes reperfusion. Treatment with streptokinase had no significant effect on haemodynamic changes or the incidence of arrhythmias between the groups. Streptokinase did, however, reduce total left ventricular infarct size in the non-reperfused and reperfused groups: $35 \%$ (group II, non-reperfused) $v 45 \%$ (group I, nonreperfused controls) $(p<0.001)$, and $15 \%$ (group IV, streptokinase and reperfusion) $v 25 \%$ (group III, reperfused controls) ( $p<0.01)$.

These results show that streptokinase inhibits the early development of necrosis in the presence of a persistent coronary artery occlusion.

\section{Prediction of coronary patency: abrupt electrocardiographic changes versus ventricular late potentials}

\section{E W Chew, P Morton, M E Scott, D B O'Keeffe Cardiac Unit, Belfast City Hospital, Belfast}

The two aims of this study were (a) to compare ventricular late potentials (VLP) and abrupt changes on 12 lead electrocardiograms in predicting patency after thrombolysis (group 1) and (b) to predict patency by using VLP in the convalescent period in a group of patients not treated with thrombolysis (group 2). Coronary angiography was performed in 79 patients in group 1 and 36 in group 2 . Of the 
45 patients in group 1 with abrupt electrocardiographic changes, $42(93.3 \%)$ had patent infarct related arteries. Of the 34 who did not have these changes, $15(44 \cdot 1 \%)$ had occluded vessels. Abrupt electrocardiographic changes predicted an open artery with a specificity of $83.3 \%$ and a sensitivity of $68.9 \%$. Of the same 79 patients, 14 had VLP. Twelve $(85.7 \%)$ had occluded vessels. Of the 65 who did not have VLP, $59(90.7 \%)$ had open arteries. The absence of VLP predicted patency with a higher sensitivity $(96 \cdot 1 \%)$ but lower specificity $(66 \cdot 7 \%)$. Of the 19 patients without abrupt electrocardiographic changes and open arteries, 17 $(89.5 \%)$ did not have VLP. Almost all of this group would be correctly identified by the absence of VLP. Of the 36 patients in group 2, all 13 with VLP had occluded vessels. Of the 23 who did not have VLP, $18(78 \cdot 3 \%)$ had open arteries. The absence of VLP predicted an open artery with a sensitivity of $100 \%$ and a specificity of $72 \cdot 2 \%$. Multivariate analysis identified VLP as a more powerful predictor of patency $(p<0.0001)$ than abrupt electrocardiographic changes $(p=0.016)$.

In conclusion, the absence of VLP is a more accurate, non-invasive means of predicting patency and may be useful in identifying which patients have patent arteries and would benefit from further invasive investigations.

\section{Dynamic changes in QT dispersion during the early phase of acute myocardial infarction: relevance for ventricular fibrillation}

P D Higham, S S Furniss, J P Bourke, R W F Campbell Academic Cardiology, Freeman Hospital, Newcastle upon Tyne

Dispersion of repolarisation is widely regarded as critical in producing ventricular fibrillation after myocardial infarction yet it has been inadequately studied during the acute phase in humans. In patients taking class III antiarrhythmic drugs we have shown increased QT dispersion in those manifesting proarrhythmic effects. Increased QT dispersion is also a feature of the long QT syndrome, in which abnormalities of repolarisation are the basis of the associated ventricular arrhythmias. We studied the changes in QT dispersion in the early phase of myocardial infarction with patients with unstable angina as a control population. We studied prospectively 33 patients admitted with chest pain by using 12 lead electrocardiograms at $50 \mathrm{~mm} / \mathrm{s}$ and a digitiser based computer program. Patients with QRS prolongation or arrhythmias or taking antiarrhythmic drugs were excluded. QT dispersion was defined as QT maximum - QT minimum, values being rate corrected. Sixteen patients had acute myocardial infarction (eight anterior, eight inferior), the mean time from onset of symptoms to admission being five hours. Thirteen patients had unstable angina and four were excluded (two because of cardiomyopathy, two because of inadequate electrocardiographic data). There was no difference in QT dispersion between patients with anterior and inferior infarcts. QT dispersion was significantly greater in the patients with acute infarction (mean 86 (30) $\mathrm{ms}$ ) compared with those with unstable angina (33 $(14) \mathrm{ms})(\mathrm{p}<0.001)$. QT maximum was prolonged in those with infarction (430 (33) ms) compared with those with unstable angina $(390(27) \mathrm{ms})(\mathrm{p}<0.001)$ whereas QT minimum was unchanged (355 (31) $v 357(28) \mathrm{ms})$. In 12 patients with acute infarction a further electrocardiogram was recorded within 24 hours (mean 14). QT dispersion diminished in 11 out of 12 patients and was unchanged in one (mean reduction $35(31) \mathrm{ms}) \mathrm{p}<0.01$. Two patients with infarcts suffered ventricular fibrillation within two hours of admission, having QT dispersions of 90 and $130 \mathrm{~ms}$.

Dynamic changes in QT dispersion occur after acute myocardial infarction and may reflect the electrophysiological conditions favouring early ventricular fibrillation.

\section{Prediction of sudden death in first year after myocardial infarction from autonomic tests: influence of age}

O Odemuyiwa, T Farrell, M Malik, Y Bashir, J Poloniecki, A J Camm

Department of Cardiological Sciences, St George's Hospital Medical School, London

To refine postinfarction risk stratification we examined the influence of age on the prediction of sudden death after infarction from autonomic tests. Of 481 patients followed up for a mean of 790 days after acute myocardial infarction, $60(12.4 \%)$ died. Of the 269 young patients (aged $<60$ years), $22(8 \cdot 2 \%)$ died; $12(54.6 \%)$ of these deaths were sudden. Of the 212 older patients (aged $\geq 60$ years), 38 died $(18 \%)$; eight $(21 \%)$ of these deaths were sudden. The following relations were found between a heart rate variability index of $<160 \mathrm{~ms}$ of the baseline width of the histogram of $R R$ interval durations, exercise duration of $<3$ minutes (EX3), ventricular ectopic frequency $>10$ beats per hour (VE10) and sudden death in the two age groups. Heart rate variability index had a sensitivity of $92 \%$, a specificity of $67.8 \%$, a positive predictive accuracy of $11.7 \%$, and a risk ratio of 21.0 in young patients ( $p<0.001$ ) but a sensitivity of $62.5 \%$, specificity of $60.4 \%$, positive predictive accuracy of $6.5 \%$, and a risk ratio of only 2.4 in older patients (NS). The combination of heart rate variability index with $\mathrm{EX} 3$ or VE10 in young patients had a sensitivity of $83 \%$, a specificity of $90 \%$, a positive predictive accuracy of $27 \%$, and a risk ratio of 31.4 $(\mathrm{p}<0.001)$ but in older patients had a sensitivity of $50 \%$, a specificity of $73.6 \%$, a positive predictive accuracy of $6.7 \%$, and a risk ratio of only $2 \cdot 6$ (NS).

Sudden death accounts for a higher proportion of deaths and is more reliably predicted by autonomic tests in younger rather than older patients. Autonomic tests did not predict sudden death in older patients.

\section{Embolisation of atrioventricular node with microvascular collagen: new treatment for atrioventricular nodal tachycardia}

\section{J F Sneddon, N J Linker, S O’Nunain, I A Simpson, A J Camm, D E Ward \\ St George's Hospital, London}

Modification of the atrioventricular node by direct current or radiofrequency energy has recently been described for the treatment of atrioventricular nodal re-entry tachycardia without the need for permanent pacing. A transcoronary approach for nodal ablation has been another new development. We investigated the feasibility of transcoronary modification of the atrioventricular node by embolisation with microvascular collagen with the aim of producing limited nodal and perinodal damage. Three patients with highly symptomatic drug refractory atrioventricular nodal re-entry tachycardia underwent selective catheterisation of the atrioventricular nodal artery with a $2 \cdot 2 \mathrm{~F}$ infusion catheter. Microfibrillar collagen $2 \mathrm{mg} / \mathrm{ml}$ (Target therapeutics ANGIOSTAT) was injected over 2 
seconds $(0.1 \mathrm{ml}$ in case 3 and $0.5 \mathrm{ml}$ in cases 1 and 2$)$. All patients developed transient complete atrioventricular block. One patient (case 3) still had symptoms and subsequently underwent-electrical modification. At restudy after eight weeks the two other patients were free of inducible tachycardia and had no evidence of dual atrioventricular nodal pathways. The mean $\mathrm{AH}$ interval increased from $65 \mathrm{~ms}$ to $72 \mathrm{~ms}$, atrioventricular nodal effective refractory period from $290 \mathrm{~ms}$ to $310 \mathrm{~ms}$, and atrioventricular nodal Wenckebach cycle length from $240 \mathrm{~ms}$ to $350 \mathrm{~ms}$ (cases 1 and 2). There were no changes in any of these parameters in the remaining patient, the failure of the procedure probably being explained by the low dose of collagen used. As the natural history of such lesions is unknown, permanent pacemakers were inserted in all patients. This technique may result in ablation of the slow conducting pathway without appreciably altering the properties of the fast pathway.

Embolisation of the atrioventricular node with microvascular collagen is a new and potentially valuable technique for modification of atrioventricular nodal function in patients with atrioventricular nodal re-entry tachycardia.

Successful catheter ablation of substrate for atrial flutter with multiple direct current shocks

\author{
S O’Nunain, N J Linker, J F Sneddon, Nadia Debbas, \\ A J Camm, D E Ward \\ St George's Hospital, London
}

Atrial flutter in humans is due to a macro re-entry circuit in the right atrium with an area of slow conduction posteroinferiorly near the os of the coronary sinus. We ablated this area of slow conduction in seven patients (seven men aged 50-67) with refractory atrial flutter by using low energy direct current shocks. This was achieved in four patients-three in established atrial flutter (mean duration 17 months)-during attempted His bundle ablation. The ablation catheter was positioned to give a large atrial signal with a minimal His deflection and 3-10 shocks were delivered (cumulative energy 75-200 J). Although complete atrioventricular block was achieved in three patients initially, conduction had returned by one month. However, all four patients remained in sinus rhythm and free of arrhythmia during follow up (8-15 months). We have now prospectively attempted selective ablation of the atrial flutter substrate in three further patients with refractory atrial flutter. Twelve to 15 low energy shocks (cumulative energy 105-235 J) were delivered directly to the atrial wall at multiple sites in the triangle of Koch. At early follow up (3-5 months) two patients remained free of atrial flutter, though one developed previously undocumented atrial fibrillation.

Successful catheter ablation of atrial flutter can be achieved by multiple low energy direct current shocks.

\section{Electrophysiological effects of pharmacological load manipulation by converting enzyme inhibitors and vasodilators in patients with impaired left ventricular function}

Y Bashir, J F Sneddon, S O'Nunain, V E Paul, Sheila Gibson, D E Ward, A J Camm

St George's Hospital Medical School, London

There is growing interest in the contribution of contraction-excitation feedback to the pathogenesis of ven- tricular arrhythmias in failing hearts. The electrophysiological effects of pharmacological load manipulation by captopril and a combination of hydralazine with oral nitrate were investigated in eight patients with left ventricular dysfunction (ejection fraction $22 \%$ (8)) and recurrent ventricular tachycardia inducible by programmed electrical stimulation: Subjects were tested (not taking antiarrhythmic drugs) on three treatment regimens in random order: captopril, hydralazine-nitrate, and control (no vasodilator). Captopril and hydralazine-nitrate were dose titrated to reduce systolic pressure by $10-20 \%$. Programmed electrical stimulation was performed according to a 12 step protocol (maximum three extrastimuli). $\mathrm{Re}-$ polarisation was assessed from monophasic action potential duration (mean of 4-6 sites) and QT interval during atrial pacing at a cycle length of $500 \mathrm{~ms}$. Compared with control, hydralazine-nitrate and captopril produced similar falls in peak systolic pressure (19\% (10) and 15\% (9)), although hydralazine-nitrate caused a bigger reduction in systemic vascular resistance (39\% (22) v 24\% (17)) and pulmonary capillary wedge pressure (12 (5) v 6 (3) $\mathrm{mm} \mathrm{Hg}$ ). Hydralazine-nitrate produced no significant change in refractoriness or repolarisation compared with control. By contrast, captopril increased ventricular effective refractory period from $231(26)$ to $249(23) \mathrm{ms}(\mathrm{p}<0.02)$ and functional refractory period from $255(24)$ to $267(27) \mathrm{ms}$ $(p<0.05)$. There was also prolongation of the QT interval from 351 (28) to $358(25) \mathrm{ms}$ ( $\mathrm{p}<0.05$ ), but no corresponding change in monophasic action potential duration was seen. Inducibility of ventricular tachycardia by programmed electrical stimulation did not differ significantly with any regimen.

We conclude that the prolongation of ventricular repolarisation and refractoriness by captopril cannot be explained solely on the basis of altered ventricular loading, given the lack of change with hydralazine-nitrate. Specific autonomic and cell membrane effects of converting enzyme inhibitors may also be involved.

\section{Temperature controlled radiofrequency atrioventricular nodal ablation}

\section{A James, T J MacConnell, M Papouchado,} D Crossman, J C Pitts Crick

Department of Cardiology, Bristol Royal Infirmary, Bristol, and Department of Cardiology, Hammersmith Hospital, London

Problems with conventional high energy atrioventricular nodal ablation have led to a search for alternative means of performing this procedure. Radiofrequency energy is one of these alternatives which has many advantages over conventional techniques, such as less discomfort and less myocardial damage. However, its major drawback is the difficulty in controlling catheter tip temperature, which can lead to tissue charring. We evaluated a new temperature controlled radiofrequency ablation system in nine patients (aged 26-59 years). The condition of all of them was refractory to previous drug treatment. Arrhythmias comprised paroxysmal atrial fibrillation (three), paroxysmal atrial tachycardia (one), intranodal re-entry tachycardia (five). All had a satisfactory symptomatic result. In the four patients with atrial arrhythmias we obtained complete heart block (three) or high grade block (one) and performed pacemaker implantation. Selective partial ablation was achieved in four of the five patients with accessory nodal pathways. Sinus rhythm was maintained and no additional treatment was necessary. In one of these patients 
complete heart block was produced necessitating permanent pacing. In one patient there was a recurrence of nodal re-entry at six weeks but at a slower rate than before and now easily controlled by propafenone.

This new ablation technique seems to be successful in providing both complete and selective partial nodal ablation without significant discomfort (no patient required general anaesthesia) or subsequent complications (follow up 2-6 months).

\section{Surgical management of atrioventricular nodal re-entrant tachycardia}

N J Linker, Y Bashir, S O’Nunain, R C Saumarez, C J Garratt, M J Griffith, A J Camm, D E Ward, D J Parker

St George's Hospital, London

Surgical treatment of atriaventricular nodal re-entrant tachycardia (AVNRT) was first described in 1985. Since 1987 we have.operated on 11 patients (mean age 39 years, range 21-64; eight women) with AVNRT and documented duel atrioventricular nodal pathways. Three patients also had accessory atrioventricular connections (two: left free wall, one left posteroseptal), which were dealt :with by endocardial dissection at the time of operation, and one had a coexisting atrial tachycardia. Patients had been treated with a mean of five antiarrhythmic agents; one patient had previous electrical atrioventricular nodal modification and another had had an antitachycardia pacemaker fitted. The first two patients underwent surgical dissection of the atrioventricular node and the succeeding patients had cryosurgical lesions of $3 \mathrm{~mm}$ in diameter produced by freezing for two minutes at $-60^{\circ} \mathrm{C}$. These lesians were placed around the atrioventricular node and between the node and coronary sinus os during atrial pacing. If the atrioventricular interval increased.by more than $100 \mathrm{~ms}$ or if any higher degree of atrioventricular block occurred the cryotherapy was immediately discontinued and the area irrigated with warm saline. The mean bypass time was 102 minutes. Postoperatively one patient developed a left haemothorax requiring drainage and two patients developed chest infections. Electrophysiological studies were performed at one week; no patient had inducible tachycardia and four had no retrograde conduction. During a mean follow up of 12 months (range $1-40) 10$ out of $11(91 \%$ ) patients were free of AVNRT. Two patients developed atrial flutter and one paroxysmal atrial fibrillation and one patient underwent a His bundle ablation for atrial tachycardia. Complete atrioventricular block was produced at the time of operation in one patient and second degree atrioventricular block in one patient, both of whom were paced.

In conclusion, surgical treatment of AVNRT offers a high success rate and should be recommended for suitable patients.

\section{Low energy ablation of ventricular tachycardia}

A J Ahsan, A D Cunningham, E Rowland Royal Brompton National Heart and Lung Hospital, London

The report of the world registry for catheter ablation concludes that catheter ablation remains a treatment of last resort for ventricular arrhythmias because of poor efficacy
$(18 \%)$, a procedure mortality of $7 \%$, and mortality at one month of $24 \%$. We describe our experience with a low energy system in 11 consecutive patients with drug refractory ventricular tachycardia (VT) or poor tolerance of drugs. The mean age of these patients was 46 (range 23-75). Nine were men and two women. Four had arrhythmogenic right ventricular dysplasia, one had a normal heart with right ventricular outflow tract tachycardia (RVOT), one corrected tetralogy of Fallot, three-ischaemic heart disease (IHD), one xenograft aortic valve replacement (AV.R), and one fascicular tachycardia (FT). Endocardial activation mapping and pace mapping localised earliest activation to the left ventricle in six and to the right ventricle in five. In three (two with arrhythmogenic right ventricular dysplasia, one with ischaemic heart disease) a suitable site was not found and no shocks were delivered. Two to 23 (mean 11) shocks of 3-30 J were delivered to the remaining eight patients (in one patient over two sessions). The range of cumulative energy delivered was $16-411 \mathrm{~J}$ (mean $220 \mathrm{~J}$ ). There were no acute deaths or serious side effects. At follow up of 4-18 months (mean 11) three patients (RVOT, tetralogy of Fallot, fascicular tachycardia) had successful ablation of VT and were symptom free and not taking drugs. Ablation was unsuccessful in two (ischaemic heart disease, AVR). Modification was produced in three; two patients (ischaemic heart disease, arrhythmogenic right ventricular dysplasia) had no VT with a previously ineffective drug treatment and in one VT (arrhythmogenic right ventricular dysplasia) was eradicated for three months until a new configuration VT appeared.

This study shows that low energy catheter ablation of VT is a safe technique and that in $45 \%$ of patients permitted control of VT. Success (including modification) is higher $(80 \%)$ for ventricular tachycardias arising from the right ventricle than for that arising from the left (33\%). This may be due to a more localised substrate in the right ventricle and more difficult catheter manipulation in the left ventricle.

\section{Intimal proliferation in organ culture of human internal mammary artery}

Cathy M Holt, C Clelland, Sheila E Francis, A C Newby, G D Angelini

Department of Cardiac Surgery, University of Sheffield, Sheffield, and Department of Cardiology, University Hospital of Wales, Cardiff

Arterial intimal proliferation is an early feature of atherosclerosis. However, its progression in humans is difficult to study. We therefore attempted to develop an organ culture model of intimal proliferation using segments of human internal mammary artery (IMA). Segments of IMA were dissected free from adventitia, carefully opened out, and cultured intimal surface uppermost for 14 days at $37^{\circ} \mathrm{C}$ in RPMI 1640 containing $30 \%$ fetal calf serum. In a separate experiment segments of IMA had the endothelium removed before culture. Tissue viability was assessed by ATP to ADP ratio, endothelial coverage by scanning electron microscopy, and immunostaining for von Willebrand factor antigen and cell proliferation by autoradiography of histological sections. The ATP to ADP ratio of IMA did not significantly alter after 14 days in culture (1.6 (SEM 
$0 \cdot 2) v 2 \cdot 0(0 \cdot 2)(\mathrm{n}=10))$, indicating that tissue viability was maintained. Intimal proliferation was observed in histological sections of cultured IMA. Immunostaining revealed that these neointimal cells were weakly positive for $\alpha$ actin, suggesting a differentiation towards a type of smooth muscle cell. Autoradiography showed proliferating cells in the neointimal layer with few dividing cells in the medium. De-endothelialised IMA showed less neointimal thickening when compared with intact IMA (21 (2) $\mu \mathrm{m} v 36$ (7) $\mu \mathrm{m})(\mathrm{n}=6, \mathrm{p}<0.05)$ as well as a reduced number of dividing cells per millimetre intimal length $(4 \cdot 1(0 \cdot 6)$ per $\mathrm{mm} v 6 \cdot 7(1 \cdot 3)$ per $\mathrm{mm})(\mathrm{n}=6)$.

Thus, an organ culture model of human arterial intimal proliferation has been established. De-endothelialisation results in diminished neointimal thickening, providing evidence for an endothelial derived proliferation enhancing factor. Inhibitors of this factor may therefore present new possibilities for treatment.

\section{Activation of endothelial monocyte adhesion associated with intimal proliferation in organ cultures of human saphenous vein}

\section{J P Cooper, A C Newby \\ Department of Cardiology, University of Wales College of Medicine, Cardiff}

Endothelial activation may be a common factor mediating atherogenesis in coronary arteries and vein grafts. Intimal smooth muscle cell proliferation, which is a characteristic feature of atherogenesis, occurs in an endothelium dependent manner in organ cultures of human saphenous vein. We investigated whether increased leucocyte adhesion, a marker for endothelial activation, also occurred in these cultures. Saphenous vein obtained from patients undergoing a coronary artery bypass operation was opened out and $6 \mathrm{~mm}$ diameter discs were cut with the blade of a corneal trephine. Discs were cultured for 14 days in RPMI medium containing $30 \%(\mathrm{v} / \mathrm{v})$ fetal bovine serum, supplemented for the last 24 hours with $1 \mu \mathrm{Ci} / \mathrm{ml}$ of tritiated thymidine. Intimal proliferation was quantified from autoradiographs of $5 \mu \mathrm{m}$ transverse paraffin sections. Adhesion of $2 \times 10^{5}$ monocytes labelled with chromium -51 was measured by incubation for two hours at $37^{\circ} \mathrm{C}$, followed by washing 20 times at a medium-air interface, and was further defined by scanning electron microscopy. Culture of discs $(n=9)$ led to the development of a neointima 24 (7) $\mu \mathrm{m}$ thick, which contained $15 \cdot 4(2 \cdot 1)$ thymidine labelled cells per mm of section length. The proportion of monocytes adhering to freshly isolated vein $(7 \cdot 1 \%(1 \cdot 2), \mathrm{n}=8)$ was not affected by culturing $(6.4 \%(0.8), \mathrm{n}=8)$, but the distribution of adhering cells was altered. Monocytes adhered preferentially to endothelium denuded areas of freshly isolated vein whereas in cultured veins, where there were no endothelium denuded areas, monocytes adhered preferentially to cells with typical endothelial morphology. Adhesion of monocytes to endothelium was confirmed in transverse sections specifically stained with $U$ europaeus lectin.

Thus, monocytes adhered readily to the endothelial cells of cultured but not freshly isolated saphenous vein. This indicates that the endothelial cells responsible for stimulation of intimal smooth muscle cell proliferation are indeed activated.
$17 \beta$-Oestradiol induces endothelium independent vasodilatation in isolated sheep coronary arteries

C Jiang, M J Taggart, P M Sarrel, D C Lindsay, P A Poole-Wilson, P Collins

Department of Cardiac Medicine, National Heart and Lung Institute, London

Treatment with oestrogen reduces the risk of coronary heart disease in postmenopausal women. Protection against transient myocardial ischaemia and infarction by modulating coronary artery tone could be an important mechanism. This study assessed the relaxing effect of $17 \beta$-oestradiol on isolated sheep coronary arteries and the role of endothelium in this relaxation. Left anterior descending coronary arteries from adult male sheep were dissected free of fat and connective tissue. The endothelium was removed from alternate rings by gently rubbing the tissue with a wooden probe. Each ring (2-3 $\mathrm{mm}$ in length) was suspended in an organ bath containing modified $\mathrm{Krebs}$ solution at $37^{\circ} \mathrm{C}$, bubbled with $95 \%$ oxygen and $5 \%$ carbon dioxide. Rings were allowed to stabilise for 90 minutes under a basal tension of $1 \mathrm{~g}$. $17 \beta$-Oestradiol $(0.1,1,10 \mu \mathrm{M}$ dissolved in ethanol) or the same concentration of ethanol was added to the bath eight minutes after rings were precontracted with potassium $(30 \mathrm{mM})$, which induced comparable contractile responses in rings with and without endothelium $(1.8(0.7)$ and $1.7(0.9) \mathrm{g}$ respectively, $\mathrm{n}=6$; unpaired $t$ test, $\mathrm{p}>0 \cdot 05) .0 \cdot 1 \mu \mathrm{M} 17 \beta$-Oestradiol had no effect on precontracted rings $(p>0.05) .1$ and $10 \mu \mathrm{M} 17 \beta$-oestradiol induced significant relaxation by $33 \%$ (2) and $84 \%$ (4) in precontracted rings with endothelium and $33 \%$ (3) and $79 \%$ (6) in rings without endothelium (comparison with ethanol controls, $n=6$, $p<0.01)$. No differences were seen between rings with and without endothelium $(p>0.05)$. Furthermore, reduced haemoglobin $(10 \mu \mathrm{M})$, an inhibitor of endothelium derived relaxing factor dependent vasodilatation, and indometha$\operatorname{cin}(10 \mu \mathrm{M})$, an inhibitor of prostacyclin dependent vasodilatation, did not affect $17 \beta$-oestradiol induced relaxation.

Thus, our results indicate that $17 \beta$-oestradiol induced significant relaxation in precontracted sheep coronary arteries by an endothelium independent mechanism. These results may help to explain the beneficial effect of oestrogen replacement therapy in postmenopausal women.

\section{$17 \beta$-Oestradiol inhibits calcium currents in isolated single guinea pig cardiac myocytes}

C Jiang, P Collins, S Mochizuki, P M Sarrel, P A Poole-Wilson, K T MacLeod

Department of Cardiac Medicine, National Heart and Lung Institute, London

The negative inotropic effect of $17 \beta$-oestradiol on the myocardium from perfused rabbit heart has been described previously but the underlying mechanism is not understood. The purpose of this study was to assess the possible inhibitory effect of $17 \beta$-oestradiol on the inward calcium current in isolated single cardiac myocytes. Guinea pig ventricular myocytes isolated by enzymic perfusion were continually superfused with a modified Krebs solution containing $2.5 \mathrm{mM}$ calcium bubbled with $95 \%$ oxygen and $5 \%$ carbon dioxide at $22^{\circ} \mathrm{C}$. Microelectrodes were filled 
with either $3 \mathrm{M}$ potassium chloride or $2 \cdot 1 \mathrm{M}$ potassium citrate and $60 \mathrm{mM}$ potassium chloride and had resistances of $15-25 \mathrm{M} \Omega$. $17 \beta$-Oestradiol caused action potential duration to decrease in a dose dependent manner. Action potential duration decreased to $82.1 \%(1.6)$ in $3 \mu \mathrm{M}$ $17 \beta$-oestradiol and to $74.3 \%(8 \cdot 1)$ in $30 \mu \mathrm{M} 17 \beta$-oestradiol $(\mathrm{n}=6$, mean (SD)) within 1-3 minutes. $17 \beta$-Oestradiol also caused shape changes in the early part of the action potential, notably a more depolarised early plateau consistent with an inhibition of the calcium current. We used an Axoclamp 2-A in switch clamp mode to measure the calcium current. Cells were voltage clamped, held at $-40 \mathrm{mV}$, and subjected to step depolarisations from this voltage to elicit the calcium current. This current could be blocked by verapamil, cobalt, and cadmium and increased by isoprenaline or by increasing the external calcium concentration. After one minutes' perfusion $10 \mu \mathrm{M} 17 \beta$-oestradiol decreased the calcium current by $45 \%$ (7) without significant alteration to the shape of the current-voltage relation. The effect of $17 \beta$-oestradiol was reversible after washout for two minutes. The reversible inhibition indicated that the effect of $17 \beta$-oestradiol could not be due to run down of the calcium current.

Our results suggest that $17 \beta$-oestradiol inhibits inward calcium current and this may underlie its negative inotropic effect.

\section{Heat stress protects isolated perfused rabbit heart}

D M Yellon, E Pasini, R Ferrari, M S Marber, J $M$ Walker, D S Latchman

Cardiovascular Studies Unit, Department of Cardiology, University College Hospital, London, and Department of Cardiology, University of Brescia, Italy

Developing means of protecting the ischaemic myocardium has been the goal of cardiologists for many years. It is now realised that other than the early restoration of coronary blood flow, which is itself associated with reperfusion induced injury, little can be done by drug treatment to salvage muscle and limit the ultimate size of an evolving infarct. We investigated a new approach to protect the ischaemic and reperfused myocardium. Hyperthermia is known to induce the synthesis in the heart of a small group of proteins known as the heat shock or stress proteins, which may be able to protect the heart from a further stress. We confirmed initial protective findings associated with heat stress in rat hearts and extended this work to another species-namely, the rabbit. Rabbits $(n=8)$ were anaesthetised and body temperature raised to $42^{\circ} \mathrm{C}$ for 15 minutes (heat shock). Controls $(n=8)$ were treated in the same way but without heating. After 24 hours the animals were reanaesthetised and the hearts removed for heat shock protein analysis $(n=6)$ or perfusion with Krebs buffer. Hearts were subjected to 60 minutes of low flow ( $1 \mathrm{ml} /$ minute) ischaemia followed by 30 minutes of reperfusion and evaluated for recovery of function, creatine kinase release, tissue ATP and creatine phosphate concentrations, mitochondrial function, and glutathione release. On reperfusion hearts subjected to heat shock showed improvements in diastolic pressure $(37.1 v 53.7 \mathrm{~mm} \mathrm{Hg}$ ), post-reperfusion developed pressure $(30 \vee 18 \mathrm{~mm} \mathrm{Hg})$, creatine kinase release $(560 v 1105 \mathrm{mU} / \mathrm{min} / \mathrm{g})$, glutathione release $(0.003 v 0.4 \mathrm{nmoles} / \mathrm{min} / \mathrm{g})$, and concentrations of ATP $(6.1 v 2.8 \mu \mathrm{mol} / \mathrm{g})$ and creatine phosphate $(36.9 v$ $17.4 \mu \mathrm{mol} / \mathrm{g}$ ). In addition, the mitochondrial respiratory control index was higher in the heat shocked animals $(9 \cdot 2$ $v 5 \cdot 7)$. Western blotting showed raised 72 kilodaltons heat shock protein in the heat shocked hearts compared with controls.

These data extend the hypothesis that heat shock protects against ischaemia and reperfusion in this model. This raises the possibility of exploiting intrinsic cellular mechanisms to protect the heart from ischaemic and reperfusion damage.

\section{Intercellular adhesion molecule-1 expression on cardiac vascular endothelial cells in dilated cardiomyopathy}

Alida L P Caforio, Jessica M Mann, S H Jennison, M J Davies, G F Bottazzo, W J McKenna

St George's and Middlesex Hospital Medical Schools, London

The hyperexpression of intercellular adhesion molecule-1 (ICAM-1) and class II major histocompatibility complex antigens on vascular endothelial cells occurs in organs affected by chronic inflammation or autoimmunity, or both. ICAM-1 and class II expression on cardiac vascular endothelial cells was studied in fresh biopsy tissue from 29 patients with dilated cardiomyopathy ( $20 \mathrm{men}$, nine women, mean age 45 (11) years) and in surgical specimens from 40 control patients ( 28 men, 12 women, mean age 44 (18) years), of whom nine had congenital defects, 20 coronary artery disease, and 11 longstanding rheumatic heart disease. Double immunofluorescence was performed with monoclonal antibodies to ICAM-1 or to class II and with antiserum to human factor VIII as a vascular endothelial cell marker. Class II expression on vascular endothelial cells was more frequent in patients with dilated cardiomyopathy than in controls $(27 / 29(93 \%) v 11 / 40(27.5 \%)$, $\mathrm{p}=0.0001)$. The finding of ICAM-1 expression on vascular endothelial cells was also more common in patients with dilated cardiomyopathy than in controls $(13 / 29(45 \%) v$ $5 / 40(12.5 \%), p=0.006)$. A positive association was found between ICAM-1 and class II antigen expression; ICAM1 expression was more commonly found in class II positive tissues than in class II negative specimens $(16 / 38(42 \%) v$ $2 / 31(6 \%), p=0.002)$. Sensitivity, specificity, and predictive value of class II and of ICAM-1 molecule expression as tissue markers of dilated cardiomyopathy were $93 \%$, $72 \%, 67 \%$ and $45 \%, 87 \%, 38 \%$ respectively.

In dilated cardiomyopathy expression of class II major histocompatibility complex antigens on vascular endothelial cells is a sensitive and relatively specific marker of autoimmunity while the finding of ICAM-1 induction on cardiac vascular endothelial cells may represent a secondary response to chronic inflammation.

\section{Frequency response characteristics of intraventricular balloons used for functional assessment of isolated hearts}

C P Young, M J Shattock, M V Braimbridge, A Cave, D J Hearse

St Thomas's Hospital, London

Fluid filled intraventricular balloons have been used experimentally for many years to measure the function of isolated perfused hearts. To assess their accuracy balloons were placed in a pressure chamber containing a high qual- 
ity pressure transducer (response flat to $100 \mathrm{~Hz}$ ) and a pressure wave generated over a frequency range of $0-60 \mathrm{~Hz}$. Frequency response curves were then constructed by comparing the response of both monitoring systems with differing arrangements of balloons, tubing, and transducers to assess the contribution of each component in the system. Balloons were constructed of stretched plastic foil (about $600 \mu \mathrm{l}$ in volume), and after filling with water and air evacuation they were attached by means of polythene tubing. (100 cm long, $1 \mathrm{~mm}$ internal diameter) to a pressure transducer. Frequency response curves were generated at different balloon volumes $(30,70,100,130 \mu \mathrm{l})$. The frequency response curves of individual balloons were almost superimposable at all balloon volumes, but there were significant differences between the curves of each balloon $(p<0.05$ at $20 \mathrm{~Hz})$. The pattern of response was of amplification of the signal $(>3 \mathrm{~dB})$ between $12 \cdot 6 \cdot(3 \cdot 2) \mathrm{Hz}$, up to a maximum of $9.0(0.8) \mathrm{dB}$, and then of progressive signal attenuation ( $-3 \mathrm{~dB}$ cut off $48 \cdot 5 \cdot(9 \cdot 5) \mathrm{Hz}$ ). Changing the pressure transducer did not significantly alter the frequency response curve of each balloon. Removal of the polythene tubing from the system resulted in significantly greater resonance between 30 and $60 \mathrm{~Hz}(p<0.01)$. The results indicated that cardiac measurements of systolic and developed pressure (component frequency range $0-25 \mathrm{~Hz}$ ) would be mildly amplified but measurements of $\mathrm{dP} / \mathrm{dt}$, which focus specifically on the high frequency signals, would be severely distorted.

We conclude that, firstly, intraventricular balloons show both resonance $(12-25 \mathrm{~Hz})$ and filtering which distorts pressure measurements. Secondly, the frequency response curve of every balloon should ideally be characterised before use. Thirdly, analysis of $\mathrm{dP} / \mathrm{dt}$ is likely to be inaccurate and unreliable. Finally, the limitations of balloon measurements should be appreciated.

\section{Decision making regarding thrombolytic treatment in the community:-is the ECG useful?}

\author{
J D Gemmill, W K Lifson, A P Rae, W S Hillis, \\ F G Dunn \\ Departments of Cardiology and Medicine and
} Therapeutics, University of Glasgow, Stobhill General Hospital, Glasgow

We assessed the roles of clinical assessment and electrocardiography performed at home in decision making for thrombolytic treatment outside hospital. A total of 137 patients seen at home with chest pain requiring direct admission to the coronary care unit (CCU) were assessed prospectively on clinical grounds by their general practitioner (GP), who documented suitability for the administration of thrombolytic treatment. Patients were randomised to clinical assessment only (group 1) or clinical assessment followed by a further assessment after electrocardiography (group 2). An electrocardiogram was successfully completed for 55 patients $(76 \%)$, of which 11 were of poor quality. The decisions were compared with the prescription of thrombolytic treatment in the CCU. In group 1 thrombolytic treatment was recommended in 30 out of 82 patients, of whom 10 ultimately received thrombolytic treatment (predictive value $33 \%$ ), and not recommended in 52, of whom three received thrombolytic treatment (predictive value $94 \%$ ). In group 2 after clinical assessment alone thrombolytic treatment was recommended in 19 out of 55 patients and given to eight (predictive value $42 \%$ ) and was not recommended in 36 , of whom five received thrombolytic treatment (predictive value $86 \%$ ). The clinical assessment was reversed after electrocardiography in seven patients, incorrectly in five.

Clinical assessment alone is unreliable for the prescription of thrombolytic treatment and difficulty exists in both recording and interpretating electrocardiograms at home, thus diminishing their contribution to decision making. Means of facilitating electrocardiogram recording and interpretation are required before thrombolytic treatment can safely be administered in the community.

\section{Dundee coronary risk disk: an aid to preventive cardiology}

H Tunstall-Pedoe

Cardiovascular Epidemiology Unit, Ninewells Hospital and Medical School, Dundee University, Dundee

Coronary risk factor assessment is increasingly popular as an aid to coronary heart disease prevention. However, clinicians traditionally consider risk factors in one dimensional terms, in which low or average levels are acceptable, and action is taken if a certain level is exceeded. This has been the approach to blood pressure control and now forms the basis of cholesterol management algorithms. However, risk factors interact and clinicians are also encouraged to "take account of other risk factors" but cannot do so without a scoring system to make this possible. In collaboration with a joint committee of the Coronary Prevention Group and the British Heart Foundation a scoring system was developed incorporating smoking, blood pressure, and cholesterol concentration. A mechanical analogue, the Dundee coronary risk disk, has been produced. Its scientific basis and the results of evaluation by doctors and nurses are described, as well as its validation against the Whitehall study. It is suggested that it may not only be the yardstick for a high risk strategy in general practice but also help hospital doctors, particularly cardiologists, to assess and explain coronary risk factors.

Evidence of autonomic imbalance from analysis of heart rate variability in patients with and without exercise induced ventricular tachycardia in clinically normal hearts

J S Gill, F Lu, V Paul, D E Ward, A J Camm

Department of Cardiological Sciences, St George's Hospital Medical School, London

Spectral and non-spectral analysis of heart rate variability allows a non-invasive method of assessment of sympathetic and parasympathetic influences on the heart. Many patients with ventricular tachycardia (VT) in "clinically normal" hearts have exercise induced VT, suggesting a sensitivity to the changes in myocardial autonomic tone associated with exercise. We studied components of $\mathrm{HRV}$ in 19 patients (age range 19-60 years) with and without exercise induced VT and clinically normal hearts. 24 Hour Holter tapes obtained from patients receiving no treatment were analysed with a Marquette 8500 laser Holter system. Power spectral analysis of HRV showed that the low frequency (LF) components $(0.05-1.5 \mathrm{~Hz}$, representing parasympathetic elements) and high frequency (HF) components $(0 \cdot 15-0.4 \mathrm{~Hz}$, representing sympathetic ele- 
ments) or their ratio did not differ between patients with (low frequency $32.5(11 \cdot 8)$, high frequency 18.1 (11.3)) and without (low frequency 27.0 (11.7), high frequency 12.9 $(7 \cdot 0)$ ) exercise induced VT. Non-spectral measures of heart rate variability included the SD of normal RR intervals, root mean square successive difference (rMSSD), and the percentage of $R R$ intervals exceeding the preceding interval by $50 \mathrm{~ms}$ ( $\mathrm{pNN} 50$ ). Although the SD of normal $R R$ intervals did not differ in patients with and without exercise induced VT $(136.8(52.6) v 121 \cdot 1(34.9) \mathrm{ms})$, the rMSSD (38.7 (14.6) v 26.3 (10.3) (p=0.07)) and pNN50 $(15.5(10.8)$ v $6.1(6.4)(\mathrm{p}<0.05))$ were higher in patients with exercise induced VT. The indices rMSSD and pNN50 are sensitive measures of parasympathetic activity and the results suggest a predominance of parasympathetic tone in patients with exercise induced VT.

We conclude that these data provide evidence of sympathetic-parasympathetic imbalance over short time periods in patients with exercise induced VT in clinically normal hearts.

\section{QT interval dispersion and arrhythmic events in patients with congenital long $Q T$ syndromes: effects of $\beta$ blockade}

\author{
N J Linker, S O’Nunain, P Colonna, A J Camm, \\ D E Ward \\ St George's Hospital Medical School, London
}

Recent evidence has suggested that QT dispersion, recorded in a surface electrocardiogram, may be a predictor of the risk of arrhythmic events in patients with long QT interval syndromes (LQTS). We studied seven patients with congenital LQTS (six female, mean age 21 years, range 1 month -44 years), all of whom had documented torsade de pointes. All patients were studied while not receiving treatment and after a mean period of 38 months (range 2-116 months) of $\beta$ blockade. Good quality electrocardiograms were recorded in all patients at a paper speed of $50 \mathrm{~mm} / \mathrm{s}$. The QT and RR intervals were measured in all leads; the $\mathrm{QT}_{\mathrm{C}}$ interval for each lead and the dispersion between the minimum and maximum $\mathrm{QT}$ and $\mathrm{QT}_{\mathrm{C}}$ intervals were calculated. The mean QT dispersion with no treatment was $109(47) \mathrm{ms}\left(\mathrm{QT}_{\mathrm{C}} 0.107(0.031) \mathrm{s}^{\frac{1}{2}}\right)$. With $\beta$ blocking drugs the QT dispersion was 169 (60).ms $\left(\mathrm{QT}_{\mathrm{C}}\right.$ $\left.0 \cdot 158(0.052) \mathrm{s}^{\frac{1}{2}}\right)$. The QT and $\mathrm{QT}_{\mathrm{C}}$ dispersion before and during $\beta$ blockade was not significantly different. Seven of the eight patients had no further symptoms or documented arrhythmic events with $\beta$ blockade. The one patient who continued to experience torsade de pointes with $\beta$ blockade showed no difference in QT interval dispersion (115v $115 \mathrm{~ms})$ and a slight decrease in $\mathrm{QT}_{\mathrm{C}}$ dispersion $(0 \cdot 127 v$ $\left.0.093 \mathrm{~s}^{\frac{1}{2}}\right)$.

We conclude that patients with congenital LQTS have marked dispersion of the QT interval in a surface electrocardiogram. However, the beneficial antiarrhythmic effect of $\beta$ blockade in these patients is not reflected in a lessening of this abnormal dispersion.

\section{Diagnosis and management of vasovagal syndrome}

\author{
J M Morgan, A S Amer, Ann Ingram, A Fitzpatrick, \\ R Sutton \\ Westminster Hospital, London
}

Between 1980 and 1990, 55 patients (29 women, aged 67
(14) years; 26 men, aged 59 (13) years) received pacemakers for treatment of the cardioinhibitory malignant vasovagal syndrome. The syndrome was diagnosed if there was clear documentation of vasovagal bradycardia or positive results in a tilt test (cardioinhibition to less than 60 beats/minute) but not if there was only a vasodepressor response to tilting. Symptoms were syncope $(51(93 \%)$ patients); presyncope (three (5\%)); dizziness (one ( $2 \%)$ ). Three patients died of unrelated causes and were excluded. The remaining 52 patients were followed up for a mean of 4.7 (2-126) months. Thirty two (62\%) patients (group A) had complete abolition of symptoms with pacing; the condition of $16(31 \%)$ (group B) was improved (four with additional drug treatment) and that of four (7\%) (group C) was not improved. Tilt testing gave positive results in $43(83 \%)$ patients, $25(78 \%)$ in group A, $15(94 \%)$ in group $B$, and three $(75 \%)$ in group C. Resting or ambulatory electrocardiographic abnormalities (bradycardia, ischaemia) were identified in $21(66 \%)$ patients in group A, $12(75 \%)$ in group B, and three (75\%) in group C. Electrophysiological study identified sinus node or atrioventricular conduction disease in $11(34 \%)$ patients in group A, four $(25 \%)$ in group B, and three $(75 \%)$ in group C. Optimal pacemaker modes were: in group A DDI $27(84 \%)$ patients (all with hysteresis and DDD five (16\%) (two with hysteresis); in group B DDI $12(75 \%)$ (10 with hysteresis), DDD two $(13 \%)$ (one with hysteresis), and VVI two (13\%) (one in atrial fibrillation); in group C DDI one $(25 \%$ ) (with hysteresis) and DDD three (75\%).

Symptoms were abolished or considerably ameliorated by optimal cardiac pacing in $48(92 \%)$ patients. DDI pacing was used in $40(83 \%)$ of these (with hysteresis in 38 $(95 \%)$ ). Many had no demonstrable abnormality on electrophysiological study but most had positive results in a tilt test. Decision to pace, therefore, is not helped by the results of electrophysiological study but negative results in a tilt test do not preclude improvement by pacing if there is historical documentation of the cardioinhibitory malignant vasovagal syndrome.

\section{Exaggeration of latency after premature stimulation in abnormal myocardium}

\section{J M Morgan, A D Cunningham, D T Connelly, E Rowland \\ Royal Brompton National Heart and Lung Hospital, London}

Conduction time is known to be prolonged (latency) after premature stimulation. To investigate the effect of myocardial disease on latency we delivered increasingly premature ventricular stimuli during steady state ventricular pacing $(600 \mathrm{~ms})$ at the right ventricular apex during electrophysiological study of patients without myocardial disease (controls) and patients with myocardial disease. Onset of activation at the right ventricular apex was determined by recording monophasic action potential (MAP) upstroke. Activation time at the right ventricular apex was measured as the interval from stimulus artefact to MAP upstroke. Activation time was consistently prolonged to a maximum with approach to refractoriness and was expressed as the percentage increase from steady state value $\left(\% \mathrm{AT}_{\max }\right)$. In 10 controls mean $\% \mathrm{AT}_{\max }$ at 19 sites was $36 \cdot 1 \%$ (SEM $6 \cdot 4 \%$ ). In seven patients with ischaemic heart disease and ventricular tachycardia $\% \mathrm{AT}_{\max }$ was 
$55 \cdot 7 \%(11 \cdot 1 \%)$ at 12 sites (no significant difference from controls). In nine patients with arrhythmogenic right ventricular dysplasia, however, $\% \mathrm{AT}_{\max }$ was $76.6 \%$ $(13.3 \%)$, significantly greater $(p<0.01)$ than that in controls. In 12 patients with right and left ventricular disease (non-ischaemic) $\% \mathrm{AT}_{\max }$ was $81.9 \%(13.7 \%)$ at 24 sites, again significantly greater $(p<0.01)$ than that in controls. The mean $\% \mathrm{AT}_{\max }$ was also significantly greater than that in controls $(p<0.01)$ at 16 sites in seven patients receiving amiodarone for ventricular tachycardia complicating ischaemic heart disease $(78.7 \%(10 \%))$ and at 15 sites in four patients with arrhythmogenic right ventricular dysplasia receiving amiodarone $(81.3 \%(11.9 \%) ; \mathrm{p}<0.01)$.

In conclusion, an exaggeration of the expected increase in latency with prematurity is a notable feature of myocardium with generalised disease. A consequence of this exaggerated increase in latency will be enhanced dispersion of repolarisation. Amiodarone may also exaggerate latency but this arrhythmogenic effect could be countered by prolongation of the action potential duration.

\section{Transaortic ventricular tachycardia mapping during surgery}

\section{R C Saumarez, D J Parker, A J Camm \\ St George's Hospital Medical School, London}

Transannular left ventricular endocardial mapping during ventricular tachycardia surgery avoids a ventriculotomy but the problem remains of relating the map to anatomical structures and the precise delivery of an ablating system. We have developed a passive multijointed mechanical arm which terminates in a hand held probe that is inserted retrogradely through the aortic valve after the patient is placed on total cardiopulmonary bypass and the aorta has been incised and the coronary arteries have been cannulated and perfused. The movements of the six joints of the arm are sensed by a supervisory computer and the position of the electrode at the tip of the arm is calculated and displayed continuously on the computer monitor as a cursor over a stylised grid representing the left ventricular endocardial geometry. When electrograms are recorded their positions on the left ventricular endocardial surface are displayed on the map. The electrograms are processed to determine their activation sequences and the markers indicating their positions on the map are colour coded to display the activation pattern across the left ventricular endocardium. The points can also flash in the sequence of their activation, creating a dynamic display. The surgeon may then move the probe to any interesting point on the endocardium by aligning the cursor with the point on the map. Then the map can be refined interactively by sampling new positions that are less than $5 \mathrm{~mm}$ apart. The surgeon can, to some extent, identify the papillary muscles while exploring the left ventricular cavity and relate their positions to the positions of the electrograms recorded on the map. A case of non-ischaemic ventricular tachycardia originating in the left ventricular outflow tract, and hence inaccessible to a transmitral approach, has been mapped and successfully cryoablated with this method. We intend to extend this approach by building a cryoprobe into the tip of an exploring probe that can be attached to the arm in place of the mapping probe and placed precisely against the ventricular tachycardia focus.

\section{Dietary fat enhances survival of rejecting cardiac allografts in rats}

M P Haw, R A Forse, L Kabbash, G L Blackburn Southampton General Hospital, Southampton, and Harvard Medical School, Boston, Massachusetts, United States

Recent studies have suggested that polyunsaturated fatty acids, both $\omega-3(\mathrm{~N}-3)$ and $\omega-6(\mathrm{~N}-6)$, are immunosuppressive. This study tested the ability of Menhaden oil (MO), rich in $\omega-3$ fatty acid, and safflower oil (SO), rich in $\omega-6$ fatty acids, to prolong cardiac allograft survival. Lewis recipients and Lewis $\times$ Brown Norway donor rats were fed three test diets for six weeks. The beef tallow (BT) control diet and MO and SO diets were $20 \%$ by weight fat and varied only in fatty acid content. After six weeks Lewis recipients received an abdominal heterotopic transplant from the donor animals. Postoperatively half the animals were treated with cyclosporin at $5 \mathrm{mg} / \mathrm{kg}$ for the first 10 days; the remainder were untreated. Parameters measured were graft survival in days, animal growth, dietary fatty acid analysis, and dietary intake. Statistical analysis was by the Mann-Whitney $U$ test for non-parametric data. Animal growth and dietary intake were identical in the three dietary groups. The median graft survival and survival range were as follows: six BT grafts survived a median of $7 \cdot 5$ days (6-9), seven SO grafts $14 \cdot 3$ days (10-21), and 10 MO grafts 12.0 days $(10-14)$. Six BT and cyclosporin grafts survived 18.0 days (17-19), six SO and cyclosporin grafts 34.5 days (26-76), and six MO and cyclosporin grafts 28.5 days (24-33). The test diets SO and MO were significantly different from the BT control diet with or without cyclosporin treatment $(\mathrm{p}<0.01)$.

These data show that dietary polyunsaturated fatty acids prolong the life of rejecting cardiac allografts. It may be that such dietary fatty acids will reduce the dose of cyclosporin required for permanent allograft survival.

\section{Right heart Doppler characteristics: comparative study of live (domino) and conventional donor hearts}

D A Lythall, M B Simmonds, C Slorach, C D J Ilsley, A G Mitchell, $M \mathrm{H}$ Yacoub

Harefield Hospital, Harefield, Middlesex

The recipient hearts from patients undergoing combined heart-lung transplantation (domino hearts) provide a valuable source of donor organs for cardiac transplantation. The right ventricle of these hearts is usually hypertrophied as an adaption to high pulmonary vascular resistance (PVR). It is conceivable that after engraftment, when these hearts are exposed to normal or near normal PVR, the now inappropriate hypertrophy may impair right heart filling. Accordingly, with Doppler echocardiography right sided filling was compared in 12 patients, of whom six (heart rate 87 (SD 13) beats/minute, age 50 (10) years; time since transplant 23 (13) months; ischaemic time 102 (34) minutes; donor age 29 (7) years) had received domino (D) hearts and six (heart rate 95 (17) beats/minute; age 53 (9) years; time since transplant 7 (7) months; ischaemic time 143 (52) minutes; donor age 31 (10) years) conventional brain dead donor (ND) hearts. Superior vena cava and tricuspid flow velocities were recorded as well as mitral and 
aortic flow velocities and left ventricular dimensions and wall thickness with a Hewlett-Packard Sonos 500/1000 ultrasound system. Simultaneous phonocardiogram and respiratory tracings were recorded. No significant differences in tricuspid or superior vena cava flow velocities were observed between the two groups (peak tricuspid flow at end expiratory apnoea, D $49(9) \mathrm{cm} / \mathrm{s} v$ ND $53(13) \mathrm{cm} / \mathrm{s}$; tricuspid diastolic flow duration, D 178 (45) ms $v 165$ (38) $\mathrm{ms}$; superior vena cava systolic flow velocity, D $26(11) \mathrm{cm} / \mathrm{s} v 28(9) \mathrm{cm} / \mathrm{s}$; superior vena cava diastolic flow velocity, D $40(7) \mathrm{cm} / \mathrm{s} v 43(16) \mathrm{cm} / \mathrm{s})$. In addition, superior vena cava flow velocity integrals and duration of superior vena cava systolic and diastolic flow were similar. This suggests that right sided filling as measured by Doppler flow is not altered by the use of right heart hypertrophied donor hearts when examined at long term follow up.

\section{Heart rate and sudden death in cardiac transplant recipients}

\author{
C D Scott, J M McComb, J H Dark \\ Freeman Hospital, Newcastle upon Tyne
}

There have been three late sudden deaths in the first 104 consecutive long term survivors ( $\geq$ three months) of orthotopic cardiac transplantation at this centre. Unexplained sinus tachycardia with a rate of $\geq 130$ beats/minute had been observed in all three patients during routine follow up. We therefore retrospectively reviewed resting 12 lead electrocardiograms recorded at routine three monthly follow up and determined serial heart rates in all 104 survivors. Mean heart rates during 22 (13) months of follow up were 99.9 (16) beats/minute at three months; 100 (11.5) beats/minute at six and nine months; 101 (13) beats/minute at one year; $96.5(11.6)$ beats/minute at two years; and $94.4(10.5)$ beats/minute at three years. The mean heart rate during the follow up period was 104 (11) beats/minute. The mean heart rate was greater than the 95th centile (mean (2 SD)) in four patients, three of whom died suddenly. Postmortem examination in two of these three showed coronary artery disease. There were no other sudden deaths but four patients died of other causes. The heart rate was not related to either donor age or to rejection.

Thus, we have established a normal range for heart rate in the three years after cardiac transplantation and have shown that there is no significant change with time after transplantation. Three of the four patients with unexplained sinus tachycardia with a rate greater than the 95th centile died suddenly, compared with none of the 100 with heart rates within the usual range after transplantation. Unexplained sinus tachycardia may therefore be an adverse prognostic sign. The mechanism of such tachycardias is not known, although perhaps there is a relation with coronary artery disease.

\section{Haemodynamic characteristics of patients assessed for single lung transplantation}

J C Doig, P A Corris, C J Hilton, J H Dark, R S Bexton Academic Cardiology, Freeman Hospital, Newcastle upon Tyne

Single lung transplantation has been performed at this hospital since 1986 . Twenty five patients with end stage respiratory disease ( 14 men, 11 women; mean age 46 years, range 20-58) have undergone preoperative assessment including measurement of right heart pressures, cardiac outputs, and pulmonary resistances. Right ventricular (RV) angiography was performed in 22 and coronary arteriography in 20 . The aim of this retrospective study was to define the haemodynamic characteristics of such patients and to determine whether preoperative haemodynamic status predicted surgical outcome. Mean RV systolic pressure was $44 \mathrm{~mm} \mathrm{Hg}$ and mean pulmonary artery pressure $30 \mathrm{~mm} \mathrm{Hg}$. Pulmonary hypertension was present in 24 patients (96\%), being mild in $13(54 \%)$, moderate in six $(25 \%)$, and severe in five $(21 \%)$. Mean pulmonary artery wedge pressure was $12 \mathrm{~mm} \mathrm{Hg}$. Mean cardiac index was $3.01 / \mathrm{min} / \mathrm{m}^{2}$, mean total pulmonary resistance 11.1 Units $\times \mathrm{m}^{2}$, and mean pulmonary arteriolar resistance 6.6 Units $\times \mathrm{m}^{2}$. Only six $(27 \%)$ had normal RV function. Coronary disease was found in five patients $(20 \%)$. The length of history and severity of hypoxia did not predict the degree of pulmonary hypertension. Eleven of these 25 patients received transplants $(44 \%)$, with four dying in the first month. Survivors had more pulmonary hypertension (RV systolic pressure $53 v 41 \mathrm{~mm} \mathrm{Hg}$, mean pulmonary artery pressure $35 v 25 \mathrm{~mm} \mathrm{Hg}, \mathrm{p}=0.024)$ but better cardiac indices $\left(3.0 v 2.2 \mathrm{l} / \mathrm{min} / \mathrm{m}^{2}\right)$ with lower total pulmonary resistance $\left(12.5 v 15.3\right.$ Units $\left.\times \mathrm{m}^{2}\right)$ and pulmonary arteriolar resistance $\left(8.3 v 8.9\right.$ Units $\left.\times \mathrm{m}^{2}\right)$. They tended to be older ( $49 v 45$ years), and male patients did better in the early stages. The extent of RV dysfunction and coronary disease were similar in survivors and non-survivors. A further seven patients underwent transplantation without preoperative catheterisation.

These data define the population being assessed for single lung transplantation for end stage pulmonary disease. Pulmonary hypertension of variable severity is present in almost all and $73 \%$ have RV dysfunction. Transplantation is their only viable treatment option and the acute outcome seems to be better in those with lower pulmonary resistances despite the presence of more severe pulmonary hypertension.

\section{Superior qualities of University of Wisconsin (UW) solution for ex vivo preservation of pig heart}

\section{P S Mankad, N J Severs, R Lachno, S Rothery,} $M$ H Yacoub

National Heart and Lung Institute, London, and Harefield Hospital, Middlesex

We evaluated UW solution by comparing it with St Thomas's solution (ST) in isolated pig hearts subjected to eight hours of ischaemia ( $n=6$ in each). The hearts were perfused ex vivo with autologous blood for control and post-preservation assessment. Morphological, metabolic, and functional evaluation was performed. Left and right function as assessed by slope values of systolic and diastolic pressure-volume relations of isovolumically contracting heart was better preserved by UW solution (\% reduction: left ventricular systolic $52.4(5.5) v 17.7(6.7)(p<0.001)$, left ventricular diastolic $125.6 \quad(46.4) \quad v \quad 65.5 \quad(31.4)$ $(\mathrm{p}<0.05)$, right ventricular diastolic $112.3(48.7)$ v 40.2 $(31.3) \quad(\mathrm{p}<0.02)$ after ST and UW preservation respectively). Post-ischaemic recovery of left ventricular $\mathrm{dP} / \mathrm{dt}$ and $\mathrm{MvO}_{2}$ were significantly improved after UW preservation $(p<0.001)$. Coronary vascular resistance was well maintained in the UW group as opposed to the significant increase in the ST group $(\mathrm{p}<0.001)$. Preserva- 
tion of myocardial ATP was improved and energy charge unchanged after eight hours of ischaemia and reperfusion in the UW hearts $(p<0.01)$. Electron microscopic examination showed substantially better preservation of contractile apparatus after UW preservation.

These observations suggest that UW solution has the potential to improve the preservation of heart and possibly prolong the ischaemic period in clinical cardiac transplantation.

\section{Haemoglobin-oxygen affinity in chronic heart failure}

\author{
S W Davies, N Blair, S L Jordan, J D M Richards, \\ D P Lipkin \\ Royal Free Hospital and University College Hospital, \\ London
}

In chronic left heart failure there is an impaired cardiac output response to exercise and increased arteriovenous oxygen extraction and infra red spectroscopic studies show profound oxygen desaturation within exercising muscle. The haemoglobin-oxygen dissociation curve is an important determinant of oxygen delivery to the tissues; there are adaptive changes in haemoglobin-oxygen dissociation in chronic hypoxic lung disease and at high altitudes due to changes in red cell 2,3-diphosphoglycerate content. Adaptive changes in haemoglobin-oxygen dissociation might be important in heart failure when blood flow to exercising muscle is limited. Blood samples were obtained from 15 patients with severe heart failure (New York Heart Association grades III-IV), normal resting blood gas concentrations, and lung function of $\geqslant 75 \%$ predicted values and from 10 age matched controls. All subjects underwent maximal treadmill exercise with respiratory gas analysis and pulse oximetry. Haemoglobin-oxygen dissociation curves were measured at standard $\mathrm{pH} 7 \cdot 13$ by tonometry, and each patient's curve was characterised by $\mathbf{P}_{50}$, the partial pressure of oxygen at which the haemoglobin is $50 \%$ saturated with oxygen. Red cell 2,3-diphosphoglycerate content was measured by enzymatic assay. The haemoglobin-oxygen dissociation curve was shifted to the right in heart failure $\left(\mathrm{P}_{50} 34.1 \quad(1.4) \mathrm{mm} \mathrm{Hg} v 30.6\right.$ $(1.2) \mathrm{mm} \mathrm{Hg}$ in controls; $\mathrm{p}<0.02)$. As a result at a given $\mathrm{Po}_{2}$ of $30 \mathrm{~mm} \mathrm{Hg}$, haemoglobin would release $60 \%$ rather than $50 \%$ of its oxygen. Red cell 2,3-diphosphoglycerate concentrations in heart failure were near the upper limit of normal but not statistically greater than in controls. $P_{50}$ correlated weakly with exercise capacity as reflected by peak oxygen consumption $\mathrm{Vo}_{2} \max (\mathrm{R}=-0.37, \mathrm{p}<0.05)$ but not with the fall in oxygen saturation on exercise.

Thus in moderate to severe heart failure haemoglobinoxygen dissociation shifts so as to favour oxygen unloading in the periphery. This may be partly due to a small increase in red cell 2,3-diphosphoglycerate as an adaptation to peripheral hypoxia.

\section{Haemoglobin inhibits endothelium dependent relaxation in human coronary arteries in vivo \\ P Collins, $\mathrm{K}$ M Fox \\ Royal Brompton National Heart and Lung Hospital, London}

The importance of endothelium derived relaxing factor (EDRF) in the control of coronary vasomotor tone has only recently been recognised. Endothelium dependent vascular relaxation and the release of EDRF in vitro is now well established. However, evidence to indicate its presence and importance in vivo is only circumstantial in human coronary arteries. We investigated the role of EDRF in the control of coronary vasomotor tone with the specific inhibitor free haemoglobin $(\mathrm{Hb})$. We report the inhibition of acetylcholine induced coronary vasodilatation by free $\mathrm{Hb}$ in seven patients (six women and one man) with angiographically normal coronary arteries. After diagnostic cardiac catheterisation three minute coronary artery infusions were given as follows: acetylcholine $0.1 \mu \mathrm{M}$, acetylcholine $0 \cdot 1 \mu \mathrm{M}$ and $\mathrm{Hb} 1 \mu \mathrm{M}$, acetylcholine $0 \cdot 1 \mu \mathrm{M}$ and $\mathrm{Hb} 10 \mu \mathrm{M}$, and $1000 \mu \mathrm{g}$ isosorbide dinitrate. These substances were infused into the left anterior descending coronary artery through a 2 French infusion catheter. Quantitative coronary angiography was performed after each infusion. Acetylcholine $0 \cdot 1 \mu \mathrm{M}$ increased left anterior descending coronary artery diameter from (mean (SEM) Student's test) $2.32(0.12)$ to $2.78(0.20) \mathrm{mm}(\mathrm{p}<0.01)$. $\mathrm{Hb} 1 \mu \mathrm{M}$ and $10 \mu \mathrm{M}$ reversed this effect to $2 \cdot 11(0 \cdot 18) \mathrm{mm}$ $(\mathrm{p}<0.001)$ and $2.29(0.14) \mathrm{mm}(\mathrm{p}<0.05)$ respectively. Isosorbide dinitrate, an endothelium independent vasodilator, overcame the inhibitory effect of free $\mathrm{Hb}(1 \mu \mathrm{M}$ and $10 \mu \mathrm{M})$ causing vasodilatation $(3.04(0.24) \mathrm{mm}, \mathrm{p}<0.001)$.

We conclude that $\mathrm{Hb}$ is an inhibitor of EDRF in vivo at concentrations that have been recorded in some pathological states. It may provide a useful tool for further investigation of EDRF in this and other vascular beds in humans.

\section{Ventilatory abnormalities in chronic heart failure are improved by physical training}

P Davey, A Coats, T Meyer, S Adamopoulos, A Radaelli, Barbara Casedei, J Conway, P Sleight John Radcliffe Hospital, Oxford

In chronic heart failure $(a)$ patients are commonly limited by leg fatigue and dyspnoea, $(b)$ factors other than haemodynamic factors are important in determining the ventilatory abnormalities, and $(c)$ there are widespread skeletal muscle changes. We hypothesised that patients with chronic heart failure were physically deconditioned, particularly in their legs, and that this placed significant limitations on their exercise capacity. We examined the effect of an exercise training programme on exercise capacity and on ventilatory performance in 22 patients aged 54-77 (mean 64 years) with chronic, stable ( $>$ three months) nonoedematous heart failure of ischaemic origin (13/9 in New York Heart Association class II/III). Mean frusemide dose was $81 \mathrm{mg}$, and nine patients were receiving angiotensin converting enzyme inhibitors. Lung function clinically and by chest $x$ ray was normal. Initial tests excluded those with exercise induced arrhythmias and ischaemia and ensured reproducibility of baseline measurements. Patients were then entered in random order to a training or detraining first programme with crossover after eight weeks to the opposite arm. Training consisted of home based bicycle ergometry, 20 minutes/day for five days/week at $70-80 \%$ of their maximum heart rate. During detraining the bicycles were removed from patients' homes and they were asked to avoid exercise that induced fatigue or dyspnoea. At the end of each period they were assessed by an observer blinded to 
their training state. Assessment comprised upright bicycle exercise to exhaustion, with four minute $25 \mathrm{~W}$ stages, with gas analysis for minute ventilation (Vi), minute carbon dioxide production $\left(\mathrm{VCO}_{2}\right)$ and minute oxygen $\left(\mathrm{Vo}_{2}\right)$ consumption. Training increased exerise time from 14 to 16.4 minutes $(p<0.02)$ and peak $\mathrm{Vo}_{2}$ from 14.1 to $15.4 \mathrm{ml} / \mathrm{kg} / \mathrm{minute}(\mathrm{p}<0.02)$. Resting and matched submaximal workload minute ventilation decreased, though peak Vi was unchanged. Resting and submaximal oxygen consumption was unchanged. At most submaximal workloads carbon dioxide production decreased, though it increased at peak workload. The time taken to reach a respiratory quotient of 1 increased from 10.9 to 13.7 minutes. Respiratory pattern did not change, though there was a trend to larger tidal volumes and lower breathing rates. The slope of $\mathrm{Vi}$ plotted against the $\mathrm{VCO}_{2}$ production decreased, and the ventilatory carbon dioxide equivalent $\left(\mathrm{Vi} / \mathrm{VCO}_{2}\right.$ against workload, a measure of $\mathrm{Vi}$ normalised for carbon dioxide production and workload) started lower and reached a lower trough point later.

In chronic heart failure exercise training improves exercise capacity, lessens the hyperpnoea of exercise, and improves some markers of ventilatory efficiency.

\section{Characterisation of blood flow through healthy and stenosed mitral valves by cine magnetic resonance velocity mapping}

R H Mohiaddin, C C Manzara, P J Kilner, M Amanuma, D J Pennell, S R Underwood, D B Longmore

National Heart and Chest Hospitals, London

Magnetic resonance imaging is not limited by acoustic windows and is capable of accurate blood flow velocity measurement, but it has not been previously used to assess valvar stenosis. We measured mitral blood flow with magnetic resonance (MR) phase shift velocity mapping in 10 healthy volunteers and in seven patients with mitral stenosis. Mitral flow direction was determined from a TE $14 \mathrm{~ms}$ FEER magnitude acquisition sensitive to signal loss from turbulence. Mitral flow velocity (TE $3.6 \mathrm{~ms}$ ) was then acquired in two oblique planes, parallel and perpendicular to the axis of mitral flow. MR measurements were compared with those from Doppler echocardiography. Peak MR mitral flow velocity (mean (SD)) in early diastole was $68(12) \mathrm{cm} / \mathrm{s}$, and in atrial systole $39(10) \mathrm{cm} / \mathrm{s}$ with a ratio of $1.9(0.6)$. These agree with normal values obtained from Doppler echocardiography. In patients with mitral stenosis there was persistent flow throughout diastole, which was significantly higher than in normals. The average peak MR velocity measurement was $189(47) \mathrm{cm} / \mathrm{s}$ compared with Doppler velocimetry $204(56) \mathrm{cm} / \mathrm{s}$. The values correlated well $(r=0.91)$.

Magnetic resonance velocity mapping is a new noninvasive method for measuring blood flow velocity through the mitral valve, characterising its pattern, and calculating pressure half time. This technique is complementary to other non-invasive investigations for assessing mitral valve disease and evaluating left ventricular diastolic function particularly when adequate Doppler measurements are difficult.
In vitro evaluation of commercially available intravascular ultrasound system: comparison of ultrasound and histological appearances

M H Anderson, I A Simpson, Charlotte McCue, M J Davies, A J Camm, D E Ward

St George's Hospital Medical School, London

The sensitivity and specificity of a currently available intravascular ultrasound (IVUS) system in identifying the presence and nature of intracoronary plaque was assessed in nine $2 \mathrm{~cm}$ sections of coronary artery and one section of aortocoronary bypass graft removed from cadavers. Perfusion fixation at arterial pressure maintained their morphology. Vessels were sutured to a supporting platform through which ran a Sonicath $4 \cdot 8$ French gauge $20 \mathrm{MHz}$ ultrasound catheter connected to a Diasonics IVUS imaging system. A screw mechanism enabled the artery to be moved enabling imaging at predetermined sites. Indelible ink marked the orientation of the artery, which was then sectioned at the same level as the IVUS images. Sections were photographed with a scale and then prepared for histological examination. For the purposes of comparison each section was divided into four quadrants. Histology and IVUS findings were compared in 84 quadrants. Significant atheroma was present in 24 quadrants and IVUS successfully identified 12 of these. False positive diagnosis of atheroma was made in 12 quadrants, giving a sensitivity of $50 \%$ and a specificity of $81 \%$. Apart from highly echogenic areas of calcification no conclusions about plaque structure and content could be drawn from the IVUS images. Lumen areas measured by IVUS were larger by a factor of $2 \cdot 1$ than those measured from the photographs. The photographic technique was compared with measurements from the histology specimens and there was close agreement between the techniques (difference $<20 \%, r=0.93$ ). The discrepancy between IVUS and optical techniques in lumen size and plaque detail may be due to poor resolution of the intima by IVUS as the system accurately measured the lumen area of similar sized plastic tubes. Useful plaque characterisation in small and medium sized coronary arteries is likely to require the use of higher ultrasound frequencies.

\section{Detection of coronary disease by cross sectional} echocardiography during dobutamine stress

\section{P Mazeika, A Nadazdin, Celia M Oakley Clinical Cardiology Unit, Hammersmith Hospital, London}

Forty nine patients undergoing cardiac catheterisation for suspected coronary disease $(\mathrm{CAD})$ were studied while not taking antianginal treatment by cross sectional echocardiography during incremental dobutamine infusion (5, 10,15 , and $20 \mu \mathrm{g} / \mathrm{kg} / \mathrm{min}$, each dose for eight minutes). A 12 lead electrocardiogram (ECG) was taken each minute. Parasternal long and short axis and apical four and two chamber views were used. End points were a new wall motion abnormality (WMA), significant symptoms, ST depression or arrhythmia, target heart rate, or systolic blood pressure of $220 \mathrm{~mm} \mathrm{Hg}$. All had an exercise ECG on a different day. Coronary angiography showed CAD $(\geqslant 70 \%$ stenosis) in 35 . Fourteen patients had a WMA at baseline. No patient had distressing symptoms or important arrhythmia and none required intravenous $\beta$ blocker. The peak rate-pressure product during dobutamine was 11967 (3032), 15851 (4348), 17915 (3766), and 
18249 (3993) at $5,10,15$, and $20 \mu \mathrm{g} / \mathrm{kg} / \mathrm{min}$ respectively compared with 24921 (6604) on exercise (p < 0.01). Sensitivity and specificity of new WMAs for CAD was $63 \%$ (22/35) and 93\% (13/14) respectively compared with $49 \%$ (17/35) and $71 \%$ (10/14) for dobutamine ECG and $74 \%$ (26/35) and $71 \%(10 / 14)$ for exercise ECG. Eleven of 14 patients with three vessel disease had a positive stress echocardiogram compared with $6 / 12$ with single vessel disease; the lesion was correctly localised to left anterior descending or right coronary/circumflex territories in all six. Colour flow mapping (PRF $4 \mathrm{kHz}, 2.5 \mathrm{MHz}$ transducer) detected new transient mitral regurgitation in four patients, all with CAD; 18 had mild mitral regurgitation pre-test, making stress induced changes uninterpretable.

Dobutamine stress echocardiography is safe, well tolerated, and moderately sensitive particularly in severe CAD and can be used in those unable to exercise.

\section{Relation between cyclic variation in echo amplitude and segmental shortening in normal and abnormal hearts}

D A Lythall, R B Logan-Sinclair, C J D Ilsley, S S Kushwaha, M H Yacoub, D G Gibson Harefield Hospital, Harefield, Middlesex, and Royal Brompton National Heart and Lung Hospital, London

To investigate the relation between cardiac cycle dependent variation in reflected myocardial ultrasonography and different manifestations of myocardial contraction, regional echo amplitude (EA) was evaluated from real time cross sectional echocardiographic images in 38 patients. The method used for acquiring echo amplitude images allowed the assignment of absolute values of echo amplitude by using the parietal pericardium as an in vivo standard for the setting of ultrasound gain. The 38 patients studied consisted of 19 normal controls, 11 patients with cardiomyopathy and impaired ventricular function, and eight patients with severe pulmonary hypertension and reversed septal motion. This enabled the influence of impaired left ventricular performance and abnormal wall motion on cyclic variation of echo amplitude to be assessed. An index of cyclic variation (CVI) was measured, which was defined as (end diastolic EA - end systolic EA)/end diastolic EA. This allowed the incorporation of background levels of EA into the measurement of cyclic variation. CVI was compared with left ventricular cavity dimension, fractional shortening, septal and posterior wall thickness, systolic wall thickening, patient's age, and heart rate. Stepwise regression analysis showed systolic wall thickening to be the most significant independent variable correlated with CVI for both septum and posterior wall $(r=0.68$, $p=0.0001$, septum; $r=0.69, p=0.0001$, posterior wall). The slopes and intercepts for both regression equations were similar, suggesting that the relation between CVI and systolic wall thickening was independent of position within the myocardial wall. Subgroup analysis showed normal controls, patients with cardiomyopathy, and patients with pulmonary hypertension to have similar slopes and intercepts for their individual regression equations, suggesting that this relation is independent of left ventricular performance and abnormal wall motion.

There therefore seems to be a quantitative relation between the extent of cyclic variation of EA and the degree of segmental myocardial shortening as measured by systolic wall thickening which is not significantly influenced by location within the myocardium, left ventricular performance, or wall motion.
Transoesophageal contrast echocardiography and colour flow mapping: methods of choice for detection of shunts at atrial level

\author{
M A de Belder, L Tourikis, G Leech, A J Camm \\ St George's Hospital Medical School, London
}

The detection of shunts at atrial level is important and a reliable means of diagnosis is required. Precordial contrast echocardiography is usually performed to detect such shunts. To investigate the advantages of transoesophageal echocardiographic techniques 167 consecutive patients were studied with both precordial and transoesophageal echocardiography, by using cross sectional imaging with contrast techniques (with and without a Valsalva manoeuvre) as well as colour flow mapping. A patent foramen ovale was diagnosed in 31 patients, an atrial septal defect in 11 (seven with bidirectional shunts), and a pulmonary arteriovenous fistula in three. All right to left shunts were detected with transoesophageal contrast echocardiography. With this as a gold standard, the sensitivity of combined precordial techniques was $37 \%$ and that of transoesophageal colour flow mapping $46 \%$. All left to right shunts were detected by transoesophageal colour flow mapping. With this as a gold standard, the sensitivities of both precordial colour flow mapping and a transoesophageal negative right atrial contrast study were $27 \%$.

We conclude that transoesophageal contrast echocardiography is the echocardiographic method of choice for the detection of a right to left shunt at atrial level that cannot be excluded by a negative precordial study or a negative transoesophageal colour flow map study. A left to right shunt at this level is best detected by transoesophageal colour flow mapping.

\section{Significance of delayed recovery after digital exercise echocardiography}

G Athanasopoulos, A Marsonis, Jayshree Joshi, Celia M Oakley, P Nihoyannopoulos

Hamersmith Hospital, Royal Postgraduate Medical School, London

The aim of the study was to assess the incidence and significance of persisting regional wall motion abnormalities (RWMA) after a symptom limited treadmill exercise test (modified Bruce) with digital exercise echocardiography (DEE). We studied 39 consecutive patients with known coronary artery disease, 14 with one vessel, 21 with two vessel, and four with three vessel disease. RWMA analysis was performed with an 11 segment protocol, and a total score was provided for a semiquantitative evaluation of wall motion (akinesis $=1$, hypokinesis $=2$, normokinesis $=3$, dyskinesis $=-1$ ). Images were acquired and digitised on line before and immediately after exercise $(<1$ minute) and at five and 10 minutes into the recovery period. DEE was considered to be positive when new or worsening RWMA were detected immediately after the exercise test. Sixteen $(41 \%)$ patients had a positive DEE (5/14 with one vessel, $9 / 21$ with two vessel, and $2 / 4$ with three vessel disease). In $7 / 16(43 \%)$ patients RWMA were still present at five minutes and in three out of $16(19 \%)$ at 10 minutes into recovery. None of the patients with persistent RWMA at five and 10 minutes had single vessel disease, whereas three patients with two vessel and one with three vessel disease had early (< five minutes) normalisation. None of the 23 patients with negative DEE developed late RWMA. Patients with delayed RWMA 
recovery had similar exercise performance and wall motion score $(27.5(1.5)$ delayed $v 27.4(1.6)$ early) immediately after exercise to those of patients who had an early recovery.

In conclusion, persistent RWMA during recovery occurred in $43 \%$ of patients with positive DEE and were related to the presence of multivessel coronary artery disease.

\section{Intravascular ultrasound evaluation of interventional procedures}

\section{H Anderson, I A Simpson, A J Camm, D E Ward St George's Hospital Medical School, London}

To evaluate the clinical use of intravascular ultrasound (IVUS) we studied six patients undergoing invasive cardiological procedures (three angioplasty, one atherectomy, one aortic valvoplasty, one coronary angiography). An 0.014 high torque floppy guide wire was positioned in the region of interest. A $20 \mathrm{MHz}$ Meditech 4.8 French Sonicath was advanced over the guide wire and connected to a Diasonics intravascular ultrasound imaging system. The tip of the catheter must be filled with sterile water before use. All patients received 5000 units of heparin. Images were recorded on videotape and imaging was repeated after the interventional procedures. Satisfactory imaging was obtained in two patients in whom atheroma was visualised before and after angioplasty (one) and atherectomy (one). Failure to image was due to failure to cross a coronary stenosis (two), excessive movement of catheter (during valvoplasty), and failure of one imaging catheter due to incorrect filling of its tip. Several problems need to be addressed before the more widespread use of this technique can be advocated. The imaging plane of this catheter is $3.5 \mathrm{~cm}$ behind the tip because of the monorail design rendering imaging of distal vessels impossible, and fluid filling of the catheter tip is a delicate and time consuming operation. Although we were able to identify atheroma on the images, higher ultrasound frequencies will be needed to obtain useful information about intracoronary plaque composition.

IVUS allowed observation of the distribution and gross structure of plaques within the coronary arteries, although catheter movement prevented useful imaging of the aortic valve. In our experience the technique is safe and practical but further developments in catheter design are required before useful clinical data can be obtained on a regular basis.

\section{Spontaneous variability of left ventricular function: assessment with a miniature nuclear probe (Cardioscint)}

\author{
P Broadhurst, X J Liu, D Hinge, P Cashman, \\ E B Raftery, A Lahiri \\ Department of Cardiology, Northwick Park Hospital, \\ Harrow, Middlesex
}

Nuclear probes have been used for detecting rapid changes in left ventricular function, yet the normal, spontaneous variability in such measurements made at the bedside has yet to be clearly defined. Nine normal volunteers (eight men) of mean age 49 (SD 10) years underwent radionuclide ventriculography in the semisupine position. This was performed both with a $\gamma$ camera and the Cardioscint, a nonimaging miniature nuclear probe, in randomised order. The camera recorded four consecutive images, each acquisition taking a mean of $7 \cdot 3$ minutes. The probe, with a $20 \mathrm{~s}$ gated acquisition time, recorded left ventricular function for about 35 minutes per patient. The mean ejection fraction of the group was $53 \%$ (7)\% with the camera and $54 \%$ (5) $\%$ with the probe. When the variability in ejection fraction measured by the camera was compared with that measured by the probe averaged over corresponding time periods-that is, an average of 7.3 minutes-their mean coefficients of variation were similar $(3.9 \%$ with the camera and $3.1 \%$ with the probe). Twenty second probe variability over the total acquisition time was also satisfactory with a coefficient of variation of $5 \cdot 4 \%$. In only two patients was this index of short term variability significantly greater than that of the camera despite the camera's longer acquisition time.

Thus the probe provides a stable recording of ejection fraction. These "normal" coefficients of variation may now provide a basis for the definition of pathological deviations.

\section{Magnetic resonance assessment of aortic flow during dobutamine stress predicts extent of myocardial ischaemia}

D J Pennell, S R Underwood, C C Manzara, D N Firmin, R H Swanton, J M Walker, P A Poole-Wilson, P J Ell, D B Longmore Magnetic Resonance Unit, Royal Brompton National Heart and Lung Hospital and Middlesex Hospital, London

In patients with coronary artery disease an abnormal response of global left ventricular function to stress is diagnostic and predicts prognosis. We examined the value of cine magnetic resonance (MR) velocity mapping during dobutamine stress in the assessment of parameters of aortic blood flow as indicators of ischaemia related ventricular dysfunction. We studied ascending aortic flow in five normal volunteers and 25 patients, 22 of whom had coronary artery disease. The extent of ischaemia was assessed by dobutamine/thallium myocardial perfusion tomography. MR imaging was performed at rest and during each of four stages of a dobutamine infusion from $5-20 \mu \mathrm{g} / \mathrm{kg} / \mathrm{minute}$. Stroke volume, cardiac output, peak flow, and peak flow acceleration were derived from the instantaneous flow measurements through the cardiac cycle. Between baseline and peak stress the change in peak flow acceleration was higher in controls than in patients with coronary artery disease $(19.8(3.5) v 10.8(4 \cdot 1), \mathrm{p}<0.001)$. The change in peak flow was also higher in controls $(23 \cdot 1$ (3.4) $v 16 \cdot 3(6 \cdot 6)$, $\mathrm{p}<0.01)$ but considerable overlap occurred between the two groups. Changes in stroke volume and cardiac output were not significantly different between the groups. There was an inverse linear relation between the increase in peak flow acceleration and the extent of myocardial ischaemia. A fall in peak flow acceleration during stress occurred only in patients with moderate or severe ischaemia $(p<0.001)$.

A failure to increase peak flow acceleration by $151 / \mathrm{s}^{2}$ identified patients with coronary artery disease with $86 \%$ sensitivity and $86 \%$ specificity. The changes in peak flow acceleration predicted the extent of reversible ischaemia. $A$ reduction in peak flow acceleration was indicative of moderate or severe myocardial ischaemia. 
Isolation, culture, and characterisation of human cardiac microvascular endothelial cells

\author{
Sara A Thorne, S E Abbot, C R Stevens, A T Wood, \\ D R Blake, P G Mills \\ Inflammation Research Group, The Royal London \\ Hospital Medical College, London
}

Cardiac microvascular endothelium has been implicated in mediating myocardial hypoxic reperfusion injury. Endothelial cell heterogeneity may preclude the application of data from non-cardiac or animal endothelial cell in vitro studies to the situation in the human heart. We therefore performed the isolation, culture, and characterisation of human cardiac microvascular endothelial cells; this has not been previously described, perhaps partly because of the limited tissue availability and the fastidious nature of adult microvascular endothelial cells in culture. Fresh tissues were obtained from operative specimens (mitral valve papillary muscles, right atrial appendages (RAA), and whole cardiac explants). Endocardial and epicardial surfaces, fibrous tissue, and macroscopic blood vessels were excised. RAA endocardium was rendered non-viable by immersion in absolute alcohol followed by washing in Hank's balanced salt solution. Endothelial cells were isolated by mincing the tissue into $2 \mathrm{~mm}^{3}$ pieces and incubating with a $0.5 \%$ crude collagenase solution containing $100 \mathrm{mM}$ calcium chloride (to ensure non-viability of contaminating myocytes). Capillary segments were expressed from the digest with a flat spatula. Material was disaggregated and filtered through a $250 \mu \mathrm{m}$ mesh to remove large tissue pieces. The filtrate was centrifuged and the pellet resuspended in culture medium and incubated at $37^{\circ} \mathrm{C}$. After 24 hours the cells were washed and the medium replaced, thus removing debris and leaving the adherent endothelial cells. Confluent cultures were passaged every 10-14 days. The endothelial nature of the cultured cells was confirmed by electron microscopy, by characteristic granular staining for von Willebrand factor, and by staining with the endothelial cell specific monoclonal antibody QB-END/10. To ascertain the optimal site for endothelial cell extraction, source tissues were also immunostained with the above endothelial cell markers. Morphometric studies demonstrated that all tissues contained high endothelial cell densities. However, sections from the left ventricle were found to contain a significantly greater density of microvascular endothelial cells (mean $486 \mathrm{~mm}^{2}$ ) than either RAA (mean $288 \mathrm{~mm}^{2}$ ) or papillary muscle (mean $\left.336 \mathrm{~mm}^{2}\right)(\mathrm{p}<0.05$ with a Student's $t$ test). Thus when available left ventricular myocardium will be used to gain the maximum endothelial cell yield.

\section{Ultrasonic tissue characterisation for detecting acute cardiac rejection in humans} D A Lythall, D G Gibson, R B Logan-Sinclair,
C J D Ilsley, A G Mitchell, M H Yacoub

Harefield Hospital, Harefield, Middlesex, and Royal Brompton National Heart and Lung Hospital, London

Myocardial echo amplitude was measured in 81 patients 12-2939 days (mean 473 (SD 575) days) after orthotopic cardiac transplantation. Patient age was $15-65$ years $(44 \cdot 4$ $(12 \cdot 3)$ years). A total of 105 echo amplitude studies were performed within 24 hours of right ventricular endomyocardial biopsy. Echo amplitude was determined by analysis of long axis parasternal cross sectional echocardiographic images acquired with a $3.5 \mathrm{MHz}$ mechanical sector scanner. Each image was standardised by setting the master gain to display the posterior parietal pericardium at the highest level of echo amplitude and using linear depth compensation at $2 \mathrm{~dB} / \mathrm{cm}$. For analysis selected video frames were transferred from tape to a frame store and echo amplitude determined in areas of interest in the basal septum and left ventricular wall with the help of a computer. In 25 studies acute cardiac rejection was confirmed by histology. End diastolic myocardial echo amplitude was markedly and significantly increased in both the interventricular septum and posterior wall during acute rejection (septum, 9.7 (2.9) $\mathrm{dB} v 5.6(2.0) \mathrm{dB}$; posterior wall, $10 \cdot 0(2 \cdot 8) \mathrm{dB} v 7 \cdot 8(2 \cdot 2) \mathrm{dB} ; \mathrm{p}<0.001)$. With a value of end diastolic echo amplitude in the septum of $6.9 \mathrm{~dB}$ as a marker of rejection, the sensitivity of this technique for detecting rejection was $94 \%$ and its specificity $79 \%$. We suggest that this increase in myocardial echo amplitude is related to altered acoustic properties within the myocardium associated with the increased myocardial water content that occurs during acute cardiac rejection.

These data support the usefulness of myocardial echo amplitude as a non-invasive marker of cardiac rejection and show that it is possible to measure echo amplitude with modified commercially available echocardiographic equipment.

\section{Magnetic resonance screening of cardiac} transplant rejection by $T 2$ parametric map of the left ventricle: correlations with endomyocardial biopsy

\section{J S Gill, G J Hunter, J B Bingham, M J Graves, P Graves, J Pepper}

Department of Cardiological Sciences, St George's Hospital Medical School and Department of Radiology, Guy's Hospital, London

This study evaluates the applicability of magnetic resonance imaging screening of cardiac transplant recipients as an alternative to serial right ventricular biopsy assessments of rejection. The myocardial pattern of $\mathrm{T} 2$ relaxation times was determined in eight patients who had received a cardiac transplant at least three months previously and in three control subjects. A $1.5 \mathrm{~T}$ system was used with aquisition in a parasaggital oblique section, yielding a short axis view of the ventricle midway between the apex and base. Each patient had undergone endomyocardial biopsy within five days of the magnetic resonance imaging (MRI) scan. The mean segmental $\mathrm{T} 2$ value in the control subjects was $37 \cdot 2(7 \cdot 8) \mathrm{ms}$. Two patients had segmental $\mathrm{T} 2$ values in the normal range $(<52.8 \mathrm{~ms}$ ) with no evidence of rejection on biopsy. Three patients had unequivocally raised $T 2$ values $(>58 \mathrm{~ms})$ in some segments of the left ventricle, with biopsy positive evidence of rejection. Two of these underwent repeat MRI after treatment of rejection, and the MRI maps returned to normal, as did the results on biopsy. Three patients had partial segmental T2 elevation with normal results on biopsy. These abnormal segments were distant from the biopsy sites and would be consistent with the patchy nature of the cardiac rejection process as observed in pathological studies.

We conclude that MRI may be a useful tool for the serial assessment of cardiac rejection, thereby reducing the rate and risks of invasive right ventricular biopsy. This method may also provide a more complete picture of the rejection process than has previously been possible. 
Low phosphocreatine to ATP ratio detected in vivo in failing human hypertrophied myocardium by phosphorus-31 magnetic resonance spectroscopy

\section{A Conway, J Allis, R Ouwerkerk, B Rajagopalan, G K Radda \\ MRC Biochemical and Clinical Magnetic Resonance Unit, John Radcliffe Hospital, Oxford}

Non-invasive detection of deterioration in cardiac metabolism would be valuable in deciding on the timing for surgery in patients with myocardial hypertrophy due to aortic valve disease. Phosphorus-31 magnetic resonance spectroscopy was therefore used to examine 17 patients, seven of whom complained of symptoms of heart failure due to either aortic stenosis (10) or aortic incompetence (seven). Spectra were collected by using a 1.9 Tesla $60 \mathrm{~cm}$ bore magnet and myocardial energy state was assessed from the ratio of the phosphocreatine (PCr) to ATP concentration. The left ventricular free wall thickness was similar in both groups (aortic stenosis, $1.6(0.4) \mathrm{cm} v$ aortic incompetence, $1.4(0.4) \mathrm{cm}$; NS). Seven patients (ejection fraction $37 \cdot 7 \%(17 \%))$ were taking diuretics for symptoms of heart failure (New York Heart Association class I/II). The ratio of PCr to ATP in these patients $(0.93(0 \cdot 16))$ was lower than that in controls $(\mathrm{n}=13,1.5(0.2) ; \mathrm{p}<0.001)$ or in those who were not taking diuretics (1.52 (0.2); $\mathrm{p}<0.001)$. The ratio of phosphodiester to ATP $(0.86$ $(0 \cdot 2))$ was not significantly different from that in controls $(0 \cdot 7(0 \cdot 16))$.

These findings indicate that the ratio of PCr to ATP is a sensitive marker of impairment in cardiac metabolism in patients with myocardial hypertrophy who require treatment for fluid retention. The role of this index in determining the optimum time for aortic valve replacement is being examined.

\section{Long term prognostic significance of total ischaemic burden and silent ischaemia in patients with unstable angina stabilised with medical treatment}

\section{G M C Rosano, F Romeo, V Rosano, E Martuscelli, A Reale \\ Department of Cardiology II, University of Rome; Catania; Vibo Valentia}

The aim of this study was to assess the long term prognostic significance of total ischaemic burden and silent ischaemia in 76 patients with unstable angina stabilised with medical treatment. All patients underwent Holter monitoring for at least 48 hours, beginning during the first 12 hours after hospitalisation. Total ischaemic burden $\geqslant 60$ minutes was present in 43 patients (group A) while 33 patients (group B) had a total ischaemic burden $\leqslant 60$ minutes. Over $78 \%$ of the ischaemic episodes of patients in group $A$ and $62 \%$ of those of patients in group B were silent $(p<0.05)$; nine patients in group $A$ and six in group $B$ had only silent episodes. Patients in group A frequently showed three vessel disease $(65 \% v 18 \%, \mathrm{p}<0.01)$, angiographical findings of subocclusion of the coronary arteries (grade TIMI I) $(76.7 \%$ v $42.4 \%, p<0.01)$, and ischaemic alterations in a resting electrocardiogram $(51.2 \% v 30.3 \%$, $p<0.05)$. During six years of follow up 15 patients in group $\mathrm{A}$ and eight patients in group $\mathrm{B}$ experienced myocardial infarction $(p<0.01)$; nine patients in group $A$ and four in group $B$ required coronary artery surgery $(p<0.05)$ and 10 patients in group $A$ and four in group $B$ died from cardiac causes $(p<0.001)$. The Cox's proportional function analysis showed three vessel disease to be the most important predictor of cardiac mortality and morbidity $(p=0.025)$, followed in predictive power by a total ischaemic burden $\geqslant 60$ minutes. The presence of silent ischaemia was not an independent predictor of long term morbidity and mortality.

In conclusion, patients with unstable angina and total ischaemic burden $\geqslant 60$ minutes have frequently silent ischaemic episodes on Holter monitoring, a greater extent of coronary atherosclerosis, and ischaemic alterations in the resting electrocardiogram. The long term prognosis of patients with unstable angina stabilised with medical treatment depends on the extent of coronary atherosclerosis and total ischaemic burden but not on the presence of silent ischaemia.

\section{Adjuvant xamoterol or metoprolol for control of amiodarone resistant ventricular tachycardia in patients with impaired left ventricular function}

Y Bashir, V E Paul, M J Griffith, Sheila Gibson, D E Ward; A J Camm

St George's Hospital Medical School, London

$\beta$ Blockers may be effective in combination with amiodarone in controlling ventricular tachycardia (VT) but are often limited by their negative chronotropic and inotropic effects, particularly in patients with left ventricular (LV) dysfunction. Use of xamoterol, a partial $\beta$ agonist, may avoid some of these drawbacks. The antiarrhythmic efficacy of xamoterol and metoprolol as adjuvant treatment to amiodarone was assessed by a randomised crossover study in 19 patients with LV dysfunction (ejection fraction $<30 \%$ ) and recurrent sustained VT (incessant in four cases) despite treatment with amiodarone for $>2$ weeks and other drugs (2-7, median four failures). The three regimens (amiodarone, xamoterol and amiodarone, and metoprolol and amiodarone) were compared by programmed electrical stimulation and exercise testing. Five patients were unable to tolerate metoprolol and one was unable to tolerate xamoterol because of haemodynamic deterioration. The combination of xamoterol and amiodarone was more effective than amiodarone alone or metoprolol and amiodarone in controlling VT induced at programmed electrical stimulation $(p<0.02)$. Overall, xamoterol and amiodarone produced complete or partial suppression in 13 out of 19 patients with inducible VT with amiodarone alone, although there was no significant change in ventricular effective refractory period or tachycardia cycle length. Both combinations of xamoterol with amiodarone and metoprolol with amiodarone reduced peak heart rate on exercise compared with amiodarone (108 (15) and $98(16) v 128$ (19) beats/minute; $p<0.01$ ) and suppressed exercise induced VT in three out of four cases. Eleven patients were discharged taking xamoterol and amiodarone and one taking metoprolol and amiodarone. During follow up (1-24 months) recurrent VT developed in two patients within six weeks and in one patient at 11 months. The remainder were free of tachycardia.

We conclude that xamoterol is more effective and better tolerated than metoprolol as adjuvant treatment for controlling amiodarone resistant VT in patients with poor LV function. Even in patients with antitachycardia devices xamoterol and amiodarone may be of value in reducing the frequency of attacks and facilitating tachycardia recognition by rate criteria. 


\section{Predictors of induced arrhythmia suppression and long term efficacy of oral sotalol in patients with drug refractory ventricular arrhythmias}

\section{A C Rankin, L Hamilton, D Du, J Newell, H Garan, J N Ruskin, B A McGovern \\ Massachusetts General Hospital, Boston, Massachusetts,} United States

Predictors of efficacy of sotalol, a $\beta$ adrenergic receptor blocking agent with class III antiarrhythmic action, were evaluated in 161 patients with drug refractory ventricular arrhythmias. There were 138 men and 23 women aged $20-88$ (mean 61 ) years. The majority $(89 \%)$ had coronary artery disease and the mean left ventricular ejection fraction was $41 \%$ (SD13\%). Their presenting clinical arrhythmia was ventricular tachycardia (VT) in 115 , ventricular fibrillation (VF) in 28, and symptomatic non-sustained VT (NSVT) in 18. Sotalol was not used in patients with uncompensated heart failure, a left ventricular ejection fraction of less than $20 \%$, or severe bronchospasm. In 39 out of 131 patients with sustained ventricular arrhythmia induced by programmed stimulation sotalol suppressed the arrhythmia. Multiple regression analysis of clinical variables identified four factors associated with suppression of induced arrhythmia by sotalol: $(a)$ the clinical arrhythmia $(p<0.0005),(b)$ the absence of coronary artery disease $(p<0.05),(c)$ a higher left ventricular ejection fraction $(p<0.05)$, and $(d)$ a shorter cycle length of the induced arrhythmia before drug treatment $(p<0.05)$. The best multivariable model used only the arrhythmia and coronary artery disease as independent predictors of arrhythmia suppression, with a predictive accuracy of $74 \%$. For example, the probability of arrhythmia suppression by sotalol for patients presenting with VT, VF, and NSVT was $21 \%, 59 \%$, and $73 \%$ for patients with coronary artery disease and $52 \%, 92 \%$, and $96 \%$ for those without. Seventy seven patients were discharged taking long term oral sotalol. Patients were followed up for a maximum of five years (mean 18.3 (SD 15.6) months). There were five sudden deaths and 10 recurrences of non-fatal sustained arrhythmia and four patients had symptomatic NSVT. Actuarial life table analysis showed that arrhythmia free survival was $88 \%$ at six months, $78 \%$ at one year, and $65 \%$ at two years. Multivariable analysis identified persisting inducibility, clinical arrhythmia, and age as predictors of recurrence. Event free survival at two years for patients with no inducible VT or VF with sotalol treatment was $85 \%$ and for those still inducible it was $49 \%$ $(p<0.05)$. Seventeen out of $28(61 \%)$ patients with VF at presentation were treated with long term sotalol with no recurrence of arrhythmia during a mean follow up of 22 months.

In conclusion, clinical VT and coronary artery disease are predictors of persisting arrhythmia inducibility with sotalol treatment, which in turn is a predictor of arrhythmia recurrence. When inducible VT or VF is suppressed sotalol provides effective long term treatment, especially for patients presenting with VF.

\section{Surgical management of arrhythmogenic right ventricular dysplasia}

K Nimkhedkar, S S Furniss, J P Bourke, B Glenville, J M McComb, C J Hilton, R W F Campbell

Academic Cardiology, Freeman Hospital, Newcastle upon Tyne

Arrhythmogenic right ventricular dysplasia (ARVD) is a rare but important cause of ventricular tachycardia (VT), particularly in young males. Ten patients (nine men, mean age 39 years) with ARVD underwent surgery for arrhythmia control. All were refractory to medical treatment and treatment with a minimum of three drugs (mean five), including amiodarone, had failed. All had a history of syncope and six had a history of cardiac arrest; three had a family history compatible with ARVD. At operation the right ventricle (RV) was grossly diseased. Sustained monomorphic or multimorphic VT was induced and mapped intraoperatively in all patients. One patient underwent cryoablation of an arrhythmogenic focus in the upper part of the RV septum. A disarticulation procedure was performed in the remaining nine and was partial (approximately $40 \%$ of the RV free wall) in four and total (complete electrical isolation of RV from LV) in five. During mean follow up of 21 months three patients had recurrences of VT of new configuration. All were well tolerated and easily controlled by drug treatment. Four of the nine patients who underwent RV disarticulation experienced postoperative heart failure but all improved progressively with the development of paradoxical septal motion. All 10 patients were arrhythmia free and their condition was New York Heart Association functional class I/II at last review.

Surgery effectively controls VT related to ARVD. It should be recommended to patients with ARVD whose arrhythmias are posing a threat to life or are frequent despite drug treatment.

\section{Management of refractory ventricular arrhythmias: endocardial resection or implantable defibrillator?}

\section{G Kaye, W Baig, A Murday, P Kay, E J Perrins, C Cowan \\ Department of Cardiology, Leeds General Infirmary, Leeds}

During an 18 month period 20 consecutive patients, all men (mean age 62 (15) years, range 55-70) were treated with antiarrhythmic surgery or an implantable cardioverter defibrillator (ICD) for malignant ventricular arrhythmias. Nineteen patients underwent myocardial revascularisation (CAVBG or LIMA $(n=2)$ ). In addition, eight patients had map guided endocardial resection (MGER), in two of whom cryoablation was also performed. Two patients had left ventricular aneurysmectomy. Three patients died (38\%) postoperatively of pump failure. Epicardial patches were inserted in three of the five survivors, all of whom were non-inducible 10 days after operation, and there have been no recurrent arrhythmias at a mean follow up of 13 months (range 5-20). Twelve patients were selected for ICD. Two patients died postoperatively of pump failure and one patient was noninducible after CAVBG alone. Nine patients received an ICD. All patients were alive at a mean follow up of 11 months (3-17). One patient developed atrial flutter 12 months after implantation causing inappropriate defibrillation.

Although the study group is small, the results suggest that in addition to myocardial revascularisation, MGER is indicated in monomorphic VT. ICD should be reserved for cases with poor left ventricular function, infrequent 
arrhythmias, and inferior scars where endocardial resection is technically difficult.

\section{Antitachycardia pacemaker/automatic implantable cardioverter defibrillator in patients with frequently recurring ventricular tachycardia}

\section{A C Rankin, S Zaim, B A McGovern, H Garan,} J N Ruskin

Massachusetts General Hospital, Boston, Massachusetts, United States

Ventricular tachycardia which recurs frequently is a relative contraindication to implantation of an automatic implantable cardioverter defibrillator (AICD) because of multiple shocks and battery depletion. Antitachycardia pacing is an alternative treatment, but pacing may acclerate arrhythmias. An automatic implantable programmable device (Ventritex V-100) which delivered tiered therapy (antitachycardia pacing, low energy or high energy shocks) in response to detected arrhythmias was studied in 22 patients with drug refractory recurrent ventricular tachycardia. There were 20 males and two females, aged 14 to 78 (mean 61) years. Twenty had coronary artery disease. Mean left ventricular ejection fraction was $32 \%$. Noninvasive programmed stimulation after device implantation confirmed successful termination of induced ventricular tachycardia by antitachycardia pacing in 19 patients, failure of antitachycardia pacing in two, and induction of rapid ventricular tachycardia terminated by cardioversion in one. Acceleration of induced ventricular tachycardia by antitachycardia pacing was observed in four patients. During follow up of 1-13 months (mean 5.8 months) there were no deaths. Spontaneous ventricular fibrillation and ventricular tachycardia recurred in one and 16 patients, respectively. The time interval to recurrence of ventricular tachycardia ranged from three days to four months (mean 4.6 weeks) and was less than one month in 11 patients. Assessment of arrhythmias and therapy delivered was obtained by interrogation of the device and retrieval of stored intracardiac electrograms. The number of episodes of ventricular tachycardia per patient ranged from one to 150 . Six patients had more than 50 episodes within five months. Rates of spontaneous tachycardia ranged from 130 to 210 beats/minute (mean 157 beats/minute). Spontaneous ventricular tachycardia was terminated by antitachycardia pacing in 15 out of 16 patients, in $87 \%$ of episodes. Acceleration of ventricular tachycardia by antitachycardia pacing was documented in eight patients, with ventricular fibrillation in 13 episodes. In only two of these eight patients had acceleration of patients had acceleration of ventricular tachycardia by antitachycardia pacing been demonstrated at previous non-invasive programmed stimulation. Failure of antitachycardia pacing was observed in five patients, but in only one was it never successful. Eight patients received shocks for ventricular arrhythmias. Inappropriate therapy was delivered in eight patients (during sinus tachycardia in two and atrial fibrillation in five and because of sensing lead fracture in one), with five of the eight receiving inappropriate shocks. Failure to detect ventricular tachycardia occurred in two patients because the tachycardia rate was below the detection rate.

In conclusion, antitachycardia pacing is effective in terminating ventricular tachycardia in patients with frequently recurring arrhythmia. Acceleration to life threatening arrhythmias, which require high energy shocks, may not be predicted by responses to induced arrhythmia.
Recognition of ventricular tachycardia by intracardiac electrogram cross phase spectra

\author{
R C Saumarez, V Paul, A J Murphy, A J Camm \\ St George's Hospital Medical School, London
}

A mathematical theory of ventricular tachycardia (VT) recognition for use in implantable devices has been developed that separates electrograms into components determined by the activation sequence and components due to action potential (AP) shape which may be discarded. The delays in depolarisation of individual myocytes result in a linear phase shift with frequency in the Fourier series (FS) of their APs. The FS of the recorded electrogram can be calculated by summation of the FS of all the APs in the electrode receptive field after weighting their amplitudes to account for their distances from the electrode and intervening tissue resistances and geometry. The difference in the phase of the FS of electrograms at two sites in the ventricle, the cross phase spectrum, depends on the relative delays in activation of myocytes in the two receptive fields and is independent of the AP morphology, provided that its dispersion within the electrode receptive field is small. This gives a theoretical basis for using the cross phase spectrum to detect the changes in activation between sinus rhythm and VT. Right ventricular bipolar electrograms were obtained in 24 episodes of VT in 21 patients with ischaemic heart disease and cardiomyopathy (three spontaneous, eight exercise induced, and 13 programmed stimulation), during exercise in 12 patients and over 24 hours in nine. The cross phase spectrum in sinus rhythm and during VT fell into separable distributions in 22 episodes. Studies of exercise induced and long term variability of the cross phase spectrum show that an adaptive template is required as the use of a static sinus rhythm template to detect VT would result in more than $50 \%$ non-specificity.

The cross phase spectrum is a highly sensitive method of detecting activation sequence changes during VT, but the physiological variability of intracardiac electrograms requires that adaptive methods of detecting abrupt changes in the cross phase spectrum are implemented to avoid false positive detection of VT.

\section{Heterotopic heart transplantation in ischaemic heart disease: role of adjunctive surgery to recipient heart}

P D Ridley, A Khaghani, F Musumeci, R Favaloro, $S$ Akl, N Banner, A G Mitchell, M Yacoub Department of Cardiac Surgery, Harefield Hospital, Harefield, Middlesex

The role of heterotopic cardiac transplantation in coronary heart disease has not been defined. Between 1983 and 1988 a group of 28 patients with end stage ischaemic heart disease was managed by heterotopic heart transplantation and adjunctive surgery to the recipient heart (coronary artery bypass grafts and aneurysmectomy 20 , coronary artery bypass grafts five, and aneurysmectomy three). Indications were feasibility of operative procedures to the recipient heart and small donor size ( $54 \%$ of the donors $<15$ years). There were six deaths. The one year and five year actuarial survival was $79 \%$ and $63 \%$. Of the 22 patients who survived to two years of follow up, all of whom were severely limited (New York Heart Association grade III/IV) preoperatively, 20 were in categories I or II at two year follow up $(p<0.0001)$. Only one experienced angina at two years compared with 15 preoperatively $(p<0.001)$. In 14 out of 
$22(64 \%)$ patients the recipient heart augmented the donor cardiac output significantly, and in four the recipient heart supported the patient when the donor heart failed to eject. The coronary artery bypass graft patency was $79 \%(23 / 29)$ at two years and $50 \%(8 / 16)$ at five years. Thromboembolic complications were rare (transient ischaemic attacks in two patients). The incidence of accelerated coronary artery disease in donor hearts was $9 \%(2 / 22)$ at two years and $36 \%$ (4/11) at five years.

In conclusion, this series shows the efficacy of heterotopic transplantation combined with surgery to the recipient heart in managing patients with end stage ischaemic heart disease.

\section{$\beta$ Adrenoceptor density in donor heart: a guide to prognosis?}

\section{R Chester, D B Barnett, M Yacoub}

National Heart and Lung Institute, London; Harefield Hospital, Middlesex; and Leicester University

Department of Pharmacology, Leicester

Early intractable cardiac failure unrelated to rejection is a recognised complication of orthotopic cardiac transplantation. Donor hearts are usually obtained from brain dead subjects dying in situations in which myocardial damage is known to occur via excess activation of the sympathetic adrenergic pathway. $\beta$ Adrenoceptor density down regulation is related to excess catecholamine exposure and a reduction in the density seems to contribute to the reduced physiological responsiveness of the failing heart. To test whether there is a relation between $\beta$ adrenoceptor density in the donor heart and the outcome for the recipient we retrospectively examined 31 donors not requiring inotropic support in whom right ventricular endomyocardial biopsy specimens had been obtained immediately before implantation. Radioligand binding saturation isotherms with 125-iodopindolol were performed on homogenate preparations and receptor density was determined with a non-linear regression curve fitting programme. Total $\beta$ adrenoceptor density in the donor heart biopsy specimens where the recipient developed cardiac failure immediately after transplantation and died in the perioperative month was significantly lower than that in the successful donors where the recipient survived for at least six months $(14$ (2) fmol/mg protein $(\mathrm{n}=8)$ v $22(1.5) \mathrm{fmol} / \mathrm{mg}$ protein $(\mathrm{n}=23), \mathrm{p}<0.05$ Mann-Whitney-Wilcoxon).

This study suggests that evaluation of $\beta$ adrenoceptor density in the donor myocardium may help to identify hearts associated with a poor surgical outlook.

\section{Hypomagnesaemia and myocardial magnesium depletion after cardiac transplantation}

Teri Millane, Jessica M Mann, S Devarajan, D W Holt, W J McKenna

Department of Cardiological Sciences, St George's

Hospital Medical School, London

Hypomagnesaemia is associated with cyclosporin used after organ transplantation. There are no data pertaining to the magnesium $(\mathbf{M g})$ state of cardiac transplant recipients. Prospectively we studied 12 consecutive patients who underwent cardiac transplantation between January and
June 1990. Serum and myocardial Mg content were measured at the time of endomyocardial biopsy at two weeks and three months after transplantation. In the six patients who received transplants earlier in the year measurements were repeated at six months. Myocardial $\mathrm{Mg}$ was measured in a single myocardial biopsy specimen by atomic absorption spectrometry and expressed as $\mu \mathrm{mol} \mathrm{Mg}$ per gram dry weight myocardium. Serum $\mathrm{Mg}$ fell significantly from a mean (SD) of $0.74(0.12)$ at two weeks after transplantation to $0.61(0.08) \mathrm{mmol} / \mathrm{l}$ at three months (p $<0.002$; paired $t$ test). At three months $50 \%$ of patients were significantly hypomagnesaemic (serum $\mathrm{Mg}<0.60$ ). One patient developed disabling neurological signs of $\mathbf{M g}$ deficiency (blood cyclosporin was within the therapeutic range), which responded dramatically to treatment with intravenous $\mathbf{M g}$ sulphate. Myocardial $\mathrm{Mg}$ concentrations were similarly affected, with a mean myocardial $\mathrm{Mg}$ of $32 \cdot 2(4 \cdot 7) \mu \mathrm{mol} / \mathrm{g}$ at two weeks falling to 29.4 (3.9) at three months $(\mathrm{p}<0.04)$. In two patients myocardial $\mathrm{Mg}$ concentrations were severely depressed at two weeks $(<25 \mu \mathrm{mol} / \mathrm{g})$, remaining substantially unchanged over subsequent weeks. In the patients followed up for six months mean serum $\mathrm{Mg}$ concentration had risen slightly but was still depressed at $0.65(0.09) \mathrm{mmol} / 1$ (two weeks $v$ six months; NS), with four out of six patients remaining hypomagnesaemic. There was a suggestion of further myocardial $\mathrm{Mg}$ depletion $(27.4(5.2) \mu \mathrm{mol} / \mathrm{g} ; \mathrm{p}<0.06)$, and in only one patient was the rise in serum magnesium concentration over the three to six month period paralled by an increase in myocardial $\mathrm{Mg}$ content.

We have shown that hypomagnesaemia and myocardial $\mathrm{Mg}$ depletion occur commonly in the post-transplant period. Serum $\mathrm{Mg}$ concentration tends to normalise six months after transplantation, but this is not accompanied by myocardial $\mathrm{Mg}$ repletion in most cases. Further studies are needed to investigate the clinical consequences of this observation and to establish the mechanism of $\mathrm{Mg}$ loss.

\section{Functional assessment of left ventricular performance in transplanted human hearts}

J T Stewart, I A Simpson, C Callicott, R E Smith, J R Pepper, W J McKenna

St George's Hospital Medical School, London

Denervation of the heart in animals alters myocardial substrate handling and may influence myocardial mechanical efficiency. To investigate mechanical performance in the denervated human heart, left ventricular (LV) mechanical efficiency was calculated from $\mathrm{LV}$ heat production and $\mathrm{LV}$ energy supply (oxygen utilisation) in five patients who had undergone orthotopic cardiac transplantation. This was compared with LV mechanical efficiency in a control group of eight patients with non-cardiac chest pain. The transplant recipients were male, aged 39-53 (mean 44.5) years and had undergone transplantation 1-3 (median 2) years earlier. None had clinical or histological evidence of rejection and none was taking cardioactive drugs. The control group (four men, four women) were aged 37-71 (mean 53.5) years. All patients had normal $L V$ function (ejection fraction $0.63(0.06)$ in the controls and $0.67(0.03)$ in the transplant recipients, NS) and normal epicardial coronary arteries. Energy supply to the myocardium in each group was similar $(4.4(1.7) v 4.7(0.4) \mathrm{W}, \mathrm{NS})$, but more energy was wasted as heat in the transplant recipients $(2 \cdot 4(1.0) v$ $3.6(0.4) \mathrm{W}, \mathrm{p}=0.02)$. LV mechanical efficiency was reduced in the transplant group to $24.6 \%(3.8 \%)$ compared with $44 \cdot 2 \%(9 \cdot 6 \%)$ in the controls $(p=0.001)$. 
Despite the normal clinical and angiographic findings, there is evidence that $\mathrm{LV}$ energetics are abnormal in transplanted human hearts. Whether the mechanism is due to denervation is unclear, but the reduced mechanical efficiency of the left ventricle in transplanted human hearts may have important long term clinical implications.

\section{Doppler echocardiographic studies of flow in the superior vena cava: a sensitive and specific marker of acute cardiac rejection}

\author{
M B Simmonds, D A Lythall, C Slorach, C D J Ilsley, \\ A G Mitchell, $\mathbf{M} \cdot \mathbf{H}$ Yacoub \\ Harefield Hospital, Harefield, Middlesex
}

Doppler echocardiographic studies of superior vena cava (SVC) and pulmonary vein flow have shown a significant increase in the ratio of diastolic to systolic flow in patients with myocardial restriction. As changes resembling those seen in myocardial restriction have been observed during acute cardiac rejection, SVC Doppler flow was evaluated as a marker of acute rejection after orthotopic cardiac transplantation. Eighteen patients were studied, of whom six (heart rate 104 (SD 16) beats/minute; age 54 (5) years; time since transplantation 6.3(11.7) months; ischaemic time 167 (60) minutes; donor age 31 (10) years) had histological criteria of rejection on endomyocardial biopsy performed within 24 hours. The remaining 12 patients (two women, heart rate 91 (15) beats/minute; age 51 (9) years; time since transplantation 15 (13) months; ischaemic time 122 (47) minutes; donor age 30 (8) years) were free from rejection. Pulsed wave Doppler studies of SVC flow were recorded in the supine position with a $5 \mathrm{MHz}$ transducer and a Hewlett-Packard Sonos 500/1000 ultrasound system. Simultaneous phonocardiogram and respiratory tracings were recorded. Peak flow velocities (PFV) and flow velocity integrals (FVI) of SVC systolic and diastolic forward flow were measured. In the rejection group a marked and significant fall in systolic PFV compared with that in the non-rejection group was observed during all phases of respiration $(6.8(7.7) \mathrm{cm} / \mathrm{s} v 26.8(9.8) \mathrm{cm} / \mathrm{s}(\mathrm{p} \leqslant 0.001)$ at end expiratory apnoea; $14 \cdot 1(21 \cdot 1) \mathrm{cm} / \mathrm{s} v 33.4(17 \cdot 2) \mathrm{cm} / \mathrm{s}$ $(\mathrm{p} \leqslant 0.05)$ at end inspiration; $9.4(7.6) \mathrm{cm} / \mathrm{s} v 22.1$ $(9.0) \mathrm{cm} / \mathrm{s}(\mathrm{p} \leqslant 0.02)$ at end expiration). Importantly, the pattern of SV.C flow did not seem to be affected by the timing of recipient atrial activity. If a value for SVC systolic flow velocity of $\leqslant 17 \mathrm{~cm} / \mathrm{s}$ at end expiratory apnoea is chosen as indicating rejection then sensivity was $100 \%$, specificity $75 \%$, and predictive accuracy $83 \%$.

These changes in SVC flow probably reflect restricted right ventricular filling due to myocardial oedema associated with acute rejection. Thus the examination of SVC flow provides a sensitive and specific marker of acute cardiac rejection.

\section{Demonstration of proliferating cell nuclear antigen in myocardial biopsy specimens after cardiac transplantation}

M J Davies, S Jennison, J Mann

St George's Hospital Medical School, London

Proliferating cell nuclear antigen (PCNA) is the polymerase $\delta$ accessory protein required for nuclear DNA synthesis. Specific monoclonal antibodies to PCNA can be used on histological sections to identify cells that are about to proliferate. PCNA containing cells were counted in the myocardial biopsy specimens taken in the management of cardiac transplantation. Positive marking was observed in up to $5 \%$ of myocyte nuclei, beginning with the first biopsy specimen taken at two weeks and continuing until a steady clinical state was reached. Positive nuclear marking was also seen in the interstitial connective tissue cells and in lymphoreticular cells during episodes of rejection. The number of positive cells correlates with the degree of rejection by conventional histological criteria. Severe rejection with myocytolysis was associated with positive counts of over 200 nuclei per $\mathrm{mm}^{2}$ of tissue. Initiation of treatment caused a very rapid decline in the number of positive cells and occurred before the interstitial cellularity declined. Analysis of biopsy specimens preceding an episode of rejection did not show an increase of positive cells.

The presence of PCNA positive cells is thus not a sensitive means of predicting subsequent rejection but can confirm that rejection has been controlled.

Fibrinolytic treatment for unstable angina: double blind placebo controlled trial with alteplase

M J Roberts, A J McNeill, G W N Dalzell,

D J Flannery, C M Wilson, G C Patterson, $S$ W Webb, N P S Campbell, M M Khan, A O Molajo, A A J Adgey

Regional Medical Cardiology Centre, Royal Victoria Hospital, Belfast

In patients with acute unstable angina subtotal thrombotic coronary occlusion has been described. We studied 80 consecutive patients ( 56 male, mean age 61 years) with acute angina at rest ( $\geqslant 15$ minutes) and ST segment depression of $\geqslant 1 \mathrm{~mm}$ in any electrocardiogram lead. Twenty nine patients had had a previous myocardial infarction. All patients received oral aspirin and intravenous heparin and were randomised to group A (alteplase $100 \mathrm{mg}$ ) and group B (placebo) given over three hours. Within three days of admission coronary arteriography in 37 out of 40 patients from each group showed similar significant coronary lesions ( $\geqslant 50 \%$ luminal stenosis)-eight patients with single vessel, 14 with double vessel, and 14 with triple vessel disease in group A compared with seven patients with single vessel, 13 with double vessel, and 13 with triple vessel disease in group B. Five patients had no coronary stenoses (one in group A, four in group B) and there were four left main stem stenoses (one in group $A$, three in group B). The ischaemia related vessel was patent (TIMI 2 or 3 ) in 31 out of $37(84 \%)$ patients in group A and 29 out of 37 $(78 \%)$ in group B. Myocardial infarction occurred in 26 patients in group $A$ ( 13 non-Q wave, $13 Q$ wave) and in 24 patients in group $B$ (19 non- $Q$ wave, five $Q$ wave). Thirty patients did not develop myocardial infarction. The left ventricular ejection fraction was $48 \%$ (range $17-69 \%$ ) in group A and $56 \%$ (range $31-81 \%$ ) in group B. Eight patients in each group required percutaneous transluminal coronary angioplasty and four patients in group $\mathrm{A}$ and five in group $B$ required coronary artery bypass grafting during their stay in hospital. There were seven deaths (four in group A and three in group B).

Thus in patients with unstable angina (rest pain) receiving aspirin and heparin alteplase did not significantly improve patency in the ischaemia related vessel. The requirement for percutaneous transluminal coronary angioplasty and coronary artery bypass grafting was similar in both groups. 


\section{Streptokinase resistance titre and antistreptokinase IgG do not influence fibrinolytic efficacy of streptokinase containing thrombolytic agents}

J D Gemmill, K J Hogg, J M A Burns, R Fears, R Standring, J T Douglas, G D O Lowe, A P Rae, F G Dunn, W S Hillis

Department of Medicine and Therapeutics, University

of Glasgow, Stobhill General Hospital, Glasgow

Previous streptococcal infections and treatment with streptokinase containing agents induce an antibody response that may increase resistance to future treatment with these agents. This study examines the influence of baseline antistreptokinase IgG concentrations and streptokinase resistance titres on parameters of fibrinolysis and thrombin activity. Eighty two patients (40-74 years) presenting within six hours of onset of acute myocardial infarction were treated with either $1.5 \mathrm{MIU}$ streptokinase as a 60 minute continuous intravenous infusion $(n=41)$ or 30 units of anistreplase $(n=41)$ as a five minute intravenous injection. Pretreatment streptokinase resistance titres $(n=54)$ and specific antistreptokinase IgG concentrations $(\mathrm{n}=82)$ were measured. The distributions of both parameters were skewed toward low values (streptokinase resistance titre: median 50, interquartile range 20-100 IU streptokinase $\mathrm{m}^{-1}$; antistreptokinase IgG: median 0.965, $0.472-1.903 \mu \mathrm{g}$ streptokinase binding $\mathrm{ml}^{-1}$ ). Venous blood was collected at frequent intervals from predosing to five days, immediately separated, and frozen for subsequent analysis for D-dimer and BB 15-42 fragment as indices of fibrinolysis. BB 15-42 and D-dimer release was calculated by measuring the area under the time activity curves. No significant relation between the measured area under the curve and pretreatment streptokinase resistance titre or antistreptokinase IgG was observed. In the range observed these indices of previous exposure to streptococcal protein do not inhibit the in vivo fibrinolytic action of streptokinase containing thrombolytic agents, suggesting that redosing with these agents may be possible earlier than has been recently suggested.

High concentrations of apolipoprotein (a) impair thrombolysis with alteplase in acute myocardial infarction

J A Purvis, I S Young, T R Trinick, E R Trimble, A A J Adgey

Regional Medical Cardiology Centre and Department of Chemical Pathology, Royal Victoria Hospital and Department of Chemical Pathology, Ulster Hospital, Belfast

Apolipoprotein (a) (Apo(a)) has marked structural similarity to plasminogen and has been shown to interfere with clot lysis in vitro. We have therefore assessed the relation between $\mathrm{Apo}(\mathrm{a})$ concentrations and coronary artery patency after thrombolytic treatment for acute myocardial infarction. Forty four subjects were seen within six hours of an acute myocardial infarction and were given two bolus injections of human recombinant tissue-type plasminogen activator (rt-PA, alteplase) separated by 30 minutes (total dose 70 or $100 \mathrm{mg}$ ). Coronary patency was assessed at 90 minutes by angiography and did not differ significantly between dosage regimens. Apo(a) concentrations were measured at 0 , six hours, and 24 hours with a double monoclonal antibody immunoradiometric assay. Groups were compared with the Mann-Whitney U test. Subjects with patent coronary arteries $(n=35)$ had significantly lower Apo(a) concentrations than those with occluded coronary arteries $(n=9)$ (at 0 hours $270 \mathrm{u} / 1$ median range $(15-1375)$ $v 504(65-1625), \mathrm{p}=0.028$; at six hours $236.5(15-1420) v$ $598.5(72-1400), \mathrm{p}=0.044$; at 24 hours $251.5(12-1220) v$ 539 (63-1460), $\mathrm{p}=0.041)$. Apo(a) did not change significantly in either group over the 24 hour period.

These results indicate that $\mathrm{Apo}(\mathrm{a})$ may significantly impair the thrombolytic action of rt-PA at physiological concentrations in vivo.

\section{Increased exercise capacity after nebulised diamorphine in chronic heart failure}

N G Uren, S W Davies, Sally L Jordan, D P Lipkin Department of Cardiology, Royal Free Hospital, London

To investigate whether nebulised opiate, through a peripheral action on pulmonary afferent $\mathrm{C}$ fibres, may modify the sensation of dyspnoea and improve exercise capacity in chronic left ventricular failure, eight male patients (mean age 60 years, range 52-74) in New York Heart Association class II underwent maximal treadmill exercise testing with respiratory gas analysis. After baseline exercise testing nebulised inhalations of placebo or $5 \mathrm{mg}$ diamorphine were given over 15 minutes in a randomised, double blind manner. These were delivered via a modified Acorn system nebuliser to ensure small droplet size $(1 \mu \mathrm{m})$ and peripheral airway deposition. Patients exercised immediately after nebulisation, and dyspnoea was assessed with a $100 \mathrm{~mm}$ visual analogue score. There was a significant increase in maximal oxygen consumption $\left(\mathrm{Vo}_{2} \max \right)$ in $\mathrm{ml} / \mathrm{min} / \mathrm{kg}$ from 16.7 (3.3) at baseline to $17.7(3.6)$ with diamorphine $(p<0.05)$. There was a significant increase in exercise duration from $641(191) \mathrm{s}$ at baseline to $734(175) \mathrm{s}$ with diamorphine $(p<0.05)$. There was no difference in ventilatory equivalent for carbon dioxide production slope $\left(\mathrm{VE} / \mathrm{VCO}_{2}\right)$ or in the visual analogue score at $\mathrm{a} \mathrm{Vo}_{2}$ of $10 \mathrm{ml} / \mathrm{min} / \mathrm{kg}$ or at maximal exercise with diamorphine compared with baseline.

Thus, finely nebulised diamorphine increases maximal exercise capacity and exercise duration in chronic left ventricular heart failure but with no reduction in the sensation of dyspnoea.

\section{Chronic angiotensin converting enzyme inhibition does not improve aerobic exercise capacity after myocardial infarction}

K G Oldroyd, S G Ray, M Pye, E Henderson, N McFarlane, J Christie, I Ford, S M Cobbe, H J Dargie

Departments of Cardiology, Royal and Western Infirmaries, Glasgow, and Databases Unit, University of Glasgow, Glasgow

In a double blind study 99 patients were randomised to receive placebo or captopril within 24 hours of acute myocardial infarction. Treatment was continued for one year, during which serial exercise tests were performed. The groups were well matched at baseline. At 12 months 35 patients taking captopril and 32 placebo completed symptom limited maximal cardiopulmonary treadmill exercise 
tests. Although the patients taking captopril had a significantly higher resting left ventricular ejection fraction, there was no difference between the groups in any of the following exercise parameters: peak heart rate (139 (SD 23) $v 135(24)$ beats/minute, $\mathrm{p}=0.57)$, peak systolic blood pressure (163 (25) $v 167(26) \mathrm{mm} \mathrm{Hg}, \mathrm{p}=0.61$ ), exercise time $(448(209) v 435(176) \mathrm{s}, \mathrm{p}=0.78)$, peak oxygen consumption (23.1 (6.1) $v 22.2(5.5) \mathrm{ml} / \mathrm{min} / \mathrm{kg}, \mathrm{p}=0.56)$, peak carbon dioxide production $(22.4(7 \cdot 1)$ v $22 \cdot 7$ $(7 \cdot 3) \mathrm{ml} / \mathrm{min} / \mathrm{kg}, \mathrm{p}=0.87$ ), and peak ventilation (59.1 $(20 \cdot 2) v 62.6(19 \cdot 3) 1 / \mathrm{min})$. Exercise provoked ST segment depression of at least $1 \mathrm{~mm}$ in 11 patients taking captopril and nine taking placebo. There was no difference in the time to $1 \mathrm{~mm}$ ST segment depression between the groups. Captopril was withdrawn and the patients re-exercised one month later with similar results.

We conclude that in this group of patients after infarction one year's treatment with captopril had no effect on aerobic exercise capacity.

\section{Randomised double blind placebo controlled trial of nimodipine in 1215 patients with acute stroke}

\author{
J J Murphy for TRUST Study Group \\ Department of Medicine, University Hospital, \\ Nottingham
}

Stroke is a common disease in patients with cardiac problems and both myocardial and cerebral infarction share a common pathogenesis. As with myocardial infarction, there is now much interest in cerebral salvage after stroke, the aim being to reduce mortality and improve recovery.
The trial of United Kingdom stroke treatment (TRUST) was the first British multicentre, acute intervention study on stroke and used the calcium channel blocker nimodipine, which increases cerebral blood flow and has cytoprotective properties. Seventeen centres participated in the study. Patients were entered if they were older than 40 , had become hemiparetic in the previous 48 hours, were conscious, able to swallow, and had been independent before the stroke. Those entered received nimodipine $40 \mathrm{mg}$ three times a day or matching placebo for 21 days. The primary end point was functional independence at six months, measured on an activities of daily living scale, the Barthel index. From preceding two tail power calculations 1200 patients gave greater than $90 \%$ probability at the $5 \%$ significance level of detecting a $10 \%$ difference in the proportions of patients independent with each treatment. In all 4507 patients were assessed, of whom $1215(27 \%)$ were entered. The age of patients ranged from 35 to 97 (mean 73) years. A total of 607 received nimodipine and 608 placebo, the two groups being well matched in terms of preceding fitness and stroke severity. By six months $55 \%$ of the nimodipine and $58 \%$ of the placebo group were independent, the odds ratio for independence with nimodipine being $0.88(95 \%$ confidence interval $0 \cdot 7$ to $1 \cdot 1)$. For mortality the odds ratio with nimodipine was $1.22(0.95$ to 1.57$)$. Activities of daily living and neurological scores also suggested delayed recovery in the nimodipine group at three weeks.

This study showed no evidence of a beneficial effect from nimodipine when administered within 48 hours of stroke onset. However, it does show that the lessons learnt from trials in myocardial infarction have now been translated into trials of acute teatment in stroke. TRUST was the first of such large, pragmatic studies and the next decade will see many more such trials, including thrombolysis in stroke. 